


\title{
PRELIMINARY REFERENCE DESIGN OF \\ FUSION REACTOR BLANKET EXHIBITING VERY \\ LOW RESIDUAL RADIOACTIVITY
}

Editor:

J. R. Powell

\author{
Contributors: \\ R. Benenati \\ R. Bezler \\ J. Fillo \\ O. Lazareth \\ S. Majeski \\ J. Powell \\ T. V. Sheehan
}

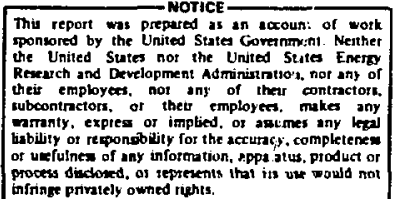

Ans: of wotk The Uniled Sting

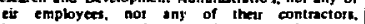
wboontrnetors, or thet employers. mikes ony bity os reponsobsty for the acruracy, completeneses



December 1, 1974

Work performed under the auspices of the U.S. Energy Research and Development Administration. 


\section{Table of contents}

Page

Summary . . . . . . . . . . . . . . . . . . . 1

1. Overview of the Low Activity Blanket Design . . . . . . 4

2. Plasma confinement . . . . . . . . . . . . . 18

3. Fuel Injection . . . . . . . . . . . . . . . . 18

4. Plasma Heating . . . . . . . . . . . . . . . 18

5. Mechanical Features of the Very Low Blanket and Shield 19

6. Neutron Activation and Breeding . . . . . . . . . . 38

7. Blanket cooling . . . . . . . . . . . . . . 50

8. Electricity Generation . . . . . . . . . . . . . 55

9. Recovery of Spent Fuel and Plasma Exhaust . . . . . . . 69

16. Recovery of Bred Tritium . . . . . . . . . . . . 70

11. Blanket Replacement and Processing . . . . . . . . . 86

12. Effluents During Normal Operation and Emergency

Containment ....................... . 100

Acknowledgements . . . . . . . . . . . . . . . 106

References

Section 1 . . . . . . . . . . . . . . 107

Section 5 ....................... . . 107

Section 6 . . . . . . . . . . . . . . 107

Section 8 ........................ . 108

Secticn 9... . . . . . . . . . . . . . . . 108

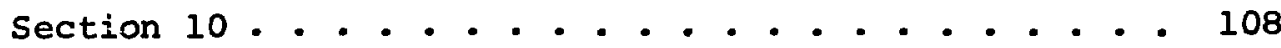

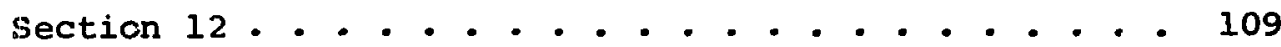

List of Tables . . . . . . . . . . . . . . . . . 110

List of Figures . . . . . . . . . . . . . . . . . 111 
Summary

The principal findings of this study are as follows:

1. The long-lived residual radioactivity in a fusion reactor blanket using aluminum structure is very low, that is, about 6 to 7 orders of magnitude lower than the inventory if one uses niobium or stainless steel structure. Short-lived activation cue to $\mathrm{Na}^{24}$ (produced by $(n, a)$ reactions on $\mathrm{Al}^{27}$ ) is on the order of $10^{9}$ curies. This isotope has a very short half life, however (15 hours). Residual radioactivity in the blanket ten days after shutdown is only $\sim 20,000$ curies. Most of this residual radioactivity results from neutron activation of small amounts of impurities in the main blanket materials: aluminum, graphite, beryllium, and aluminum oxide. This activation may be considerably reduced through further advances in purification technology. Assuming 3 years of reactor operation before shutdown and removal of the blanket, the residual radioactivity in the blanket 10 years after shutdown is only $\sim 1000$ curies, of which most is $\mathrm{Al}^{26}(740,000$ years half life).

2. Most of the blanket is discarded after 3 years operation, except for costly materials like lithium and beryllium. These are reconstituted and recycled into new blankets. The scrap has very low residual radioactivity, $\sim 1$ curie/metric ton, with no afterheat problem. It is simply compressed and stored in vaults on the reactor site. Release of activity to the environment from stored scrap should be negligible.

3. No resource problems exist for the materials (aluminum, graphite, alumina) which are discarded from the blanket. Limited resource materials (lithium and beryllium) are recycled to the blanket, and world reserves should be sufficient to permit a large fusion economy. Helium for the coolant circuit can be extracted from the atmosphere at a practical cost (i.e.., $\sim 200 / 1 b$ ) for the reactor.

4. Use of solid lithium compounds ( $\mathrm{LiAl}$ and $\mathrm{LiAlO_{2 }}$ ) for tritium breeaiing eliminates corrosion problems associated with liquid lithium and flibe. Water is used as a coolant for the aluminum shell, but at low temperature $\left(400^{\circ} \mathrm{F}\right)$ and low flow velocities $(2 \mathrm{ft} . / \mathrm{sec}$.$) .$ No corrosion problems are anticipated with either helium or water coolants. 
5. The blanket inventory of tritium can be held to relatively low levels with solid lithium compounds. With an average holdup time of tritium of 0.5 hours in the blanket, the blanket inventory of tritium is 140,000 curies. Tritium extraction from the solid compound is controlled by diffusion, and much shorter holdup times can be achieved with smaller $\mathrm{LiAl}$ and $\mathrm{LiAlO}_{2}$ particles. Particle sizes in this design are $1 \mathrm{~mm}$ for LiAl and $30 \mathrm{\mu}$ for LiAlO2. The tritium is released into the helium coolant stream, from which it is absorbed in a tritium trap consisting of a bed of titanium metal particles. The process stream, five percent of the main helium stream, flows through the tritium trap. The trap is at $500^{\circ} \mathrm{C}$, the same temperature as the process stream. After tritium is absorbed for 5 minutes, beds are switched and tritium is desorbed by cryopumping. Tritium inventory in all reactor helium circuits totals 7200 curies. The tritium trap total inventory is approximately 200,000 curies (these inventories could be substantially lower, if desired).

6. The maximum credible accident is probably the release of all the tritium in the reactor helium circuits at ground lavel. This would result in relatively small exposures $(\sim 2 \mathrm{rem})$ at the site boundary.

7. Releases of tritium during normal operation are expected to be less than 1 curie/day.

8. One-third of the blanket is replaced every year during a shutdown of one month. Replacement appears simple enough that this down time can probably be reduced one to two weeks using more working crews. Decay of $\mathrm{Na}^{24}$ in the blanket will impose a lower limit on downtime of 10 days. With minor changes in design the blanket modules should be capable of first wall loads $\sim 5 \mathrm{NW}(\mathrm{th}) /$ $\mathrm{m}^{2}$, or three times the value chosen for this design. Blankets would then have to be changed more frequently, i.e., once a year, but the downtime could be kept to a few weeks. The higher wall loads should substantially reduce the unit cost $(\$ / \mathrm{kW}(\mathrm{e}))$ of the nuclear island.

9. The overall net thermal cycle efficiency is $32 \%$. This could be increased to $\sim 34 \%$ with relatively minor design modifications, and to $\sim 36 \%$ with a graphite curtain in front of the module. Energy deposited in the low temperature aluminum structure $\left(\mathrm{T}=400^{\circ} \mathrm{F}\right)$ is used t.o raise steam which is tinen superheated by a small part of the energy deposited in the hot zone of the module. Most of the high 
quality energy is used in a high pressure steam cycle. The overall net cycle efficiency using this approach is congiderably higher than if the heat from the two temperature zones were used in separate cycles.

10. An Al-Mg-Si wrought alloy (6000 series type) is used for the structure in this design. This alloy will be more ductile and easier to fabricate than the SAP alloy proposed in previous designs.

11. The design breeding ratio is 1.20 tritions/fusion event. This should allow sufficient margin for beam hole leakage, data errors, etc. If lower breeding ratios are desired, less beryllium can be used.

12. The replacement of modules on the inner surface of the torus in the high toroidal field regions, which would be extremely difficult, is avoided by using a high temperature graphite reflector in the region. The reflector reradiates all the energy it receives by neutrons, gammas, and bremsstrahlung as thermal radiation to the remainder of the blanket. It operates at a temperature high enough $\left(\sim 1800^{\circ} \mathrm{C}\right)$ that most of the radiation damage should anneal out and cracking should not affect its performance. This region should not have to be replaced during the life of the reactor, but could be done relatively easily if required.

13. The use of water coolant for the aluminum module shells provides good temperature control and high heat transfer capability in event of plasma or beam impingement on the blanket. Water coolant is not mandatory, however, and helium coolant could be used in place of water. 


\section{Overview of the Low Activity Blanket Design}

In all fusion reactors using the deuterium-tritium fuel cycle, a large fraction ( $\$ 0 \%)$ of the fusion energy will be released as 14 MeV neutrons. These neutrons must be slowed down in a relatively shick blanket surrounding the plasma, and their kinetic energy converted to high temperature heat. This heat is continuously removed from the blanket by a coolant system. Finally, a portion of this heat is converted to electricity in a standard thermal cycle, i.e., a steam or gas turbine cycle.

The fast $14 \mathrm{MeV}$ neutrons seriously activate many candidate blanket materials. Moreover, the radiation damage resulting from these fist neutrons is a serious problem and may necessitate frequent replacement of the blanket. Thus, it is desirable that the blanket be made from materials that exhibıt little or no resicual rajibactivity. This not only greatiy eases repair, maintenance, and replacement operations, but also greatly reduces the radioactivity of damaged materials that must be handled and stored.

Prior to the blanket concept described in this study [and preceding rejposts $(1,2,3)]$ all fusion reactor reference designs used blankets: in which lithium was present as a liquid metal or salt. (For the deuterium-tritium fuel cycle, it is necessary to have lithium present in the blanket to breed tritium to replace that consumed in the plasma.) If lithium is present as liquid metal or fused salt, the only practical blanket materials that will resist corrosion by the liquid turn out to have high residual radioactivities (for example, stainless steel or niobium), and many years are required for these activated materials to decay to safe levelis. 
The lifetime of a blanket for a fusion reactor operating on DT fuel is not yet well defined, but it is clear that lifetimes will be at most a few years, rather than the 30 years or so desired fur power plants. Thus the entire blanket must be replaced a number of times during the life of a fusion plant. If stainless steel or niobium is used as the structural material, this inevitably results in handling billions of curies of activated materials during each replacement. This must be done rapidly if plant utilization factors are to be reasonably high. Furthermore, these highly radioactive materials must be stored and monitored for many years after removal.

If a solid form of lithium is used in the blanket instead of liquid lithium metal or salt, however, the choice of blanket cons'ructional materials is no longer limited by corrosion behaviour. In fact, the blanket can now have only solid components and can be cooled by an inert gas such as helium. constructional materials with very low residual activation can then be used. These materials are limited to light elements like Al, $\mathrm{Mg}, \mathrm{Si}, \mathrm{C}, \mathrm{O}$, and $\mathrm{Be}$, or compounds of those elements. Hopkins has proposed using graphite and silicon carbide as structural materials for low activity blankets: however, both materials have low ductility, and graphite undergoes large dimensional changes at relatively low fluences. Thus, such materials may not be able to reliably sustain the high stresses necessary in blanket structures. Cracks in such structures will let coolant leak into the vacuum region, and the plasma will be quenched.

Aluminum and aluminum alloys are promising structural materials for very low activity blankets. A number of conceptual blanket designs using SAP structure (aluminum strengthened by a dispersoid of very fine aluminum oxide particles), and solid lithium compounds for tritium breeding have been proposed and investigated in earlier studies $(1,2,3)$. This study concentrates 
on a specific blanket design using an Al-Mg-Si alloy as the structural material. This alloy would be similar to the 6000 series of aluminum alloys, but without constituents that can be activated (Fe, Cr, Ni, etc.). Such a wrought alloy will be much more ductile than SAP, but its maximum temperature capability is not as great as that of SAP (typically $400-500^{\circ} \mathrm{F}$ vs. $750^{\circ} \mathrm{F}$ ).

The blanket is designed to fit into the UWMAK-1 reactor (5) and to operate at the same first wall load, total thermal power, and plasma conditions.

Because of the low temperature capability of the aluminum structure, the blanket is designed with two temperature zones. The cool zone is the aluminum structure which operates at $400^{\circ} \mathrm{F}$ and is cooled by water. This structure is made of $\sim 1$ meter long cylindrical aluminum modular shells, $64 \mathrm{~cm}$ in outer diameter, and $2.5 \mathrm{~cm}$ thick, with internal channels for the water coolant. The ends facing the plasma are closed with elliptical dished heads.

The hot zone comprises the materials inside the modular aluminum shells, including graphite, beryllium, and solid lithium compounds, and it is cooled by helium with an inlet temperature of $350^{\circ} \mathrm{F}$, an exit temperature of $1400^{\circ} \mathrm{F}$, and a pressure of $20 \mathrm{~atm}$. The two zones are chermally isolated by a low conductivity insulator, i.e. low density $\mathrm{Al}_{2} \mathrm{O}_{3}$.

In the reference design described here, $32 \%$ of the total energy/fusion (including neutron absorption and gamma energy) is deposited in or leaks to the cool aluminum structure, and $68 \%$ remains in the hot zone. With design improvements, such as cooling the $\mathrm{Al}_{2} \mathrm{O}_{3}$ insulator with a radial flow of helium coolant, the portion of fusion energy deposited in or leaking to the cool zone can be reduced to $\sim 15 \%$. These improvements are discussed more fully in a later section. 
An important finding of this study is that the thermal energy from the cool zone can be converted to electrical energy at relatively high efficiency. First, a portion (i.e., several percent) of the water coolant is flashed to steam outside the blanket. The cooled water is then punped back to the aluminum modules in the blanket, and the low pressure saturated stean ( $T \sim 375^{\circ} \mathrm{F}$ ) is superheated by helium from the hot zone, and then expanded in the low pressure stages of the turbo-generator. About $10 \%$ of the thermal energy extracted from the hot zone is used for this superheat. The remaining thermal energy from the hot zone is used in a conventional high pressure steam cycle.

The net overall thermal efficiency for this combined cycle with a one-third input into the cold zone is $32 \%$. The overall efficiency could be raised to approximately $34 \%$ with the same fractional input to the cool zone by reducing overpressure on the water coolant circuit and by allowing some helium cross flow through the $\mathrm{Al}_{2} \mathrm{O}_{3}$ insulator between the hot and cuol zones.

With more substantial design modifications such as a graphite curtain that captures and re-radiates bremsstrahlung energy to a radiation sink with a temperature substantially greater than $400^{\circ} \mathrm{F}$, the net overall thermal cycle efficiency should approach " $\%$. The graphite curtain would also allow the elimination of the water coolant circuit, with the module now being cooled by a single helium coolant stream. This would simplify module connections, speed repairs, and reduce module costs.

The principal blanket mate'rials in this reference design are aluminum (with magnesium and silicon as alloying elements, graphite, aluminum oxide, lithium aluminum alloy, lithium aluminate ceramic, berylium, boron carbide, water, and helium. The available U.S. resources of aluminum (from clays), aluminum oxide, magnesium, 
silicon, graphite, water, and boron carbide can be considered virtually unlimited.

The question of lithium resources is not addressed in this study, except to note that the lithium inventory in this solid bianket design is almost two orders of magnitude smaller than that in blankets using liquid lithium metal or flibe. Iithium enriched in $\mathrm{Li}^{6}$ is used in the solid blanket design vs. natural lithium in liquid lithium blankets. The $\mathrm{Li}^{6}$ must be extracted from natural lithium; the remaining $\mathrm{Li}^{7}$ can then be used in normal chemical markets. Thus the solid blanket design minimizes impact on lithium resources, in terms of inventory, compared to liquid lithium blankets. The consumption of $\mathrm{Li}$ will be essentially the same for solid and liquid lithium blankets, however.

Helium from natural gas reservoirs is limited and may not be available when fusion reactors are being constructed. The inventory in the blanket cooling circuit is small enough $(\sim 20,000$ lbs), however, that the cost of extraction from the atmosphere is not a major part of the overall cost for the reactor. Helium resources thus can be assumed to be virtually unlimited. The question of He for the superconducting magnets is not addressed, but the He inventory for magnets will be similar to UWMAK-l.

An inventory of approximately 190 metric tons of beryllium is necessary for the blanket design. This corresponds to a total inventory of 125,000 metric tons of beryllium for an installed fusion reactor capacity of $10^{6} \mathrm{MW}(\mathrm{e})$, or approximately $20 \%$ of the projected world reserves. However, there has been relatively little exploration for beryllium, and tha actual reserves may be much larger than the above estimates. In addition, ores with low beryllium concentrations can be economically worked (6), and this should greatly increase available U. $\therefore$ and world beryllium reserves. 
Approximately 0.5 tons of $\mathrm{Be}$ is consumed per year of reactor operation or $0.1 \%$ of its inventory .

Beryllium is required for solid blanket designs if tritium breeding ratios of one or greater are to be achieved. since virtually all tritium production is by absorption of relatively slow neutrons in $\mathrm{Li}^{6}$, enough beryllium must be present that neutrons produced by $r, 2 n$ reactions in Be replace the neutrons lost by parasitic reactions with aluminum, graphite, etc. A breeding ratio of 1.20 is calculated for this reference design, which should be sufficient margin to allow for errors in data, threedimensional neutronics effects, etc. In practice, the amount of Be: in the reactor would be reduced so that the breeding ratio is only slightly greater than one, and Be inventory may, in an actual reactor, be substantially less than 190 metric tons.

The radioactive inventory at shutdown is completely dominated by $\mathrm{Na}^{24}$. This isotope has a very short half life (15 hours). however, and the initial inventory of $10^{9}$ curies decays to negligible levels (100 curies) in 10 days.

The principal activations resulting from the primary blanket materials (i.e., aluminum, graphite, oxygen, beryllium, magnesium, and silicon) are $\mathrm{Al}^{26}\left(740,000\right.$ years half 1 ife) and $\mathrm{C}^{14}(4,700$ years half life). The average inventory of these isotopes is about 600 curies in the entire blanket at any given time. since one third of the blanket is replaced every year, the average inventory of long-lived isotopes like $A I^{26}$ and $\mathrm{C}^{14}$ is approximately one half of the inventory that would be present if the entire blanket ran for 3 years before shutdown and removal.

Except for tritium, most of the long-lived blanket activation (half life of one day or greater) results from activation of impurities (e.g.. Fe, Mn, $\mathrm{Zn}$, etc.) in the primary blanket materials. 
The total impurity activation in the blanket upon shutdown is on the order of 20,000 curies. This activation will decay to negligible levels within 10 years after shutdown, and can be neglected as regards to long-term waste storage and dispersal.

The impurity levels in aluminum and aluminum oxide assumed for this design are now achieved in aluminum production plants. The cost of this aluminum is estimated as quite low-approximately $\$ 1 / 1 b$. for production in amounts appropriate to fusion reactors. Graphite of the purity level assumed in this study is currently being produced (actually much purer graphite is currently produced). The cost of graphite is estimated as approximately $\$ 2 / 1 b$ for full-scale production. The impurity level assumed for beryllium is an order of magnitude smaller than the purest material currently being produced in production plants. Total beryllium production is small, however, and there is no demand for very pure material (for fission reactor applications beryllium is satisfactory with impurity levels an order of magnitude poorer than that desired for this fusion reactor design). It seems likely that low cost purification processes can be developed, since the allowable total cost for $B e$ can be on the order of $\$ 50 / 1 \mathrm{~b}$. The most promising $\because$ vute for further reduction of blanket activation would appear to be further purification of the principal blanket materials. The impurity activation in this design seems insignificant in terms of waste disposal, since the various activities decay in a few years, and it also seems insignificant in terms of hazards. Reduction of impurity activation may make blanket repair and maintenance easier, however. Advances in materials purification technology are likely in the next few decades, and impurity activation can probably be greatly reduced by the time fusion reactors are being constructed. 
The scrap from this reactor will consist of aluminum, aluminum oxide, and graphite. The only significant long-lived activity will be $\mathrm{Al}^{26}$ (740,000 year half life). The specific activity of this waste will be very low, approximately 1 curie per metric ton. The scrap can be compressed into blocks and permanently stored in concrete vaults on the reactor site. After-hedt is completely negligible. Release to the environment should be negligible and only minimal monitoring of the stored scrap is contemplated. During the 30-year life of the plant, approximately 10,000 curies of $\mathrm{Al}^{26}$ will be generated. Considering the vast reservoir of natural aluminum in soil, even if one assumed widespread dispersion and dilution of the $\mathrm{Al}^{26}$, the resultant specific activity of soil and ground water would be many orders of magnitude below the MPC.

Tritium is bred in the solid lithium aluminum alloy in the beryllium neutron multiplication region, and also in solid lithium aluminate in the graphite neutron moderating region. The bred tritium then diffuses into the helium coolant stream, from which it is trapped out by absorption in titanium beds. Five percent of the total blaniset coolant flow is circulated through the absorption beds at a temperature of $500^{\circ} \mathrm{C}$. This process stream is extracted from the main coolant stream at $500^{\circ} \mathrm{C}$, and is returned to it at this temperature. No heat transfer is necessary for the process stream. After absorbing tritium for approximately 5 minutes, the titanium bed is switched from the process stream, and the absorbed tritium desorbed by cryopumping. The cryopumped tritium is then recycled to the fuel fabrication system, along with tritium from the plasma exhaust.

Experiments with lithium-äluminum and lithium aluminate indicate that tritium bred in it by neutrons can be readily extracted 
by diffusion into helium (7). Tritium diffusion in the solid lithium compound appears to be the controlling step in extraction, and mean holdup times of a half-hour appear readily achievable. The total tritium blanket inventory in this design is then only 140,000 curies. Smaller particle sizes for the lithium compound can reduce tritium inventory still further, if desired.

Iithium aluminum alloy (LiAl) is used in the beryllium region, since the local helium temperature is relatively low $\left(400^{\circ} \mathrm{F}\right.$ to $\left.950^{\circ} \mathrm{F}\right)$, and LiAl has a high tritium diffusion coefficient at low temperatures. The melting point of LiAl alloy is several hundred degrees above the maximum helium temperature in this region. LiAl particle sizes are on the order of several hundred microns and the LiAl pellet is composed of a loosely compacted mixture of $\mathrm{LiAl}$ particles and inert solids (e.g., Be or graphite). The pellet has sufficient open porosity that tritium will quickly escape after diffusing to the surface of a LiAl particle. If some particle attrition occurs, fines will be held in the region.

The helium temperature in the graphite region is high enough (950 to $1400^{\circ} \mathrm{F}$ ) that IiAl cannot be used. Lithium aluminate $\left(\mathrm{LiAlO}_{2}\right.$ ) is used in this design, though lithium silicate (Iisio ${ }_{3}$ ) would also be suitable, and is probably somewhat easier for extraction of tritium. LiAlO $_{2}$ particles (several tens of microns in diameter) are loosely compacted with graphite particles into pellets of approximately $1 \mathrm{~cm}$ diameter, and the pellets inserted into graphite tubes with holes to facilitate tritium escape.

The total tritium inventory in the helium coolant circuit is 7200 curies. This can be readily reduced by a factor of two by increasing the process stream flow to $10 \%$ of the total blanket flow, instead of 5\%. However, an inventory of 7200 curies seems very low, both in terms of hazards and in terms of leakage during 
normal operation. Assuming a leak rate of $1 \%$ (several times smaller than that of HTGR designs) of the helium inventory per year of operation, the plant will leak 0.22 curies/day into the primary cintainment building. Most of this txitium leakzge should be recovered by cleanup of the containment building atmosphere, so that the leak rate of tritium to the environment by this route will be $\ll 1$ curie/ day. The tritium leakage rate by diffusion through steam generator tubes is held to $\sim 1$ curie/day by using tubes with tritium diffusion barriexs (aluminum, copper, or tungsten) as proposed in other fusion reactor reference designs. This tritium will remain in the steam system. This system will be designed for zero blowdown. A small fraction of the inventory can be continuously cleaned of tritium. The tritium escape to the environment during normal operation by this route will then be substantially less than 1 curie/ day.

The helium coolant system is sectionalized into twelve separate loops. If all the helium in a one-loop system was discharged to the environment the total maximum integrated individual dose at the site boundary would be only 0.14 rem for ground level release, and 0.05 rem for release from a 50 meter stack, based on release factors developed in an AI environmental study (8). This is far less than the 25 rem maximum suggested by 10 CFR 100. Even if the helium from all 12 blanket coolant loops were completely discharged to the environment, the total integrated dose in the worst case, ground release, would still be more than an order of magnitude less than the 25 rem limit suggested by 10 CFR 100.

Release of any substantial part of the tritium in the blanket seems unlikely, since the tritium is distributed throughout the solid lithium compound. Reactor shutdown, and subsequent blanket cooldown would limit diffusion of the blanket tritium into the 
helium stream to very l.ow rates. Assuming the release of $1.0 \%$ of the 140,000 curies of blanket tritium, which seems unlikely, the total individual dose from tritium at the site boundary would be only $\sim 3$ rem for the worst: case of ground level release.

The blanket design objective has been to make replacement and repair as easy as possible, and to minimize cost. This goal has led to several decisionis embodied in this design:

1. Use of a graphite block structure on the inner part of the toroidal blanket surface.

2. Use of a number of flat surface segments with associated modules to approximate the curved toroidal surface.

3. Support of surface segments from bulkheads.

4. Installation of two module types--Type A is directly removabje together with its associated shield plug, while Type $B$ is removable through the access hole left when a Type A module is removed.

5. Use of a primary vacuum tank in which the reactor is placed, which acts as a barrier between the reactor and primary containment building.

The thickness of the blanket and shield on the inner part of the toroidal blanket should be minimized, to keep "the maximum magnet field in the superconducting windings as low as possible (maximum field is proportional to $R^{-1}$. where $R$ is the distance from the major axis of the torus. Thus it is virtually impossible to provide access between the shield and magnet in this region. one of the important results of this study is the development of a solution to this very severe problem which is common to all Tokamak reactors. In this region, a simple graphite block structure, approximately 1 meter thick, is placed in front of the 1 meter thick neutron shield. (Figure 5-1). Most of the neutron 
and bremsstrahlung energy deposited in the graphite structure is re-raciated back as thermal radiation. The graphite surface temperature is on the order of $1800^{\circ} \mathrm{C}$, with internal temperatures several hundred degrees hotter. The graphite is hot enough that radiation damage should anneal out, but not hot enough that surface evaporation poisons the plasma. The graphite block structure covers only $5 \%$ of the toroidal blanket surface. Lithium aluminate rods for tritium breeding are placed in the cooler $\left(\mathrm{T} \sim 1000^{\circ} \mathrm{C}\right)$ portions of the graphite blanket, approximately 0.5 meters from the first wall surface of the graphite. The effect of the sraphite region on the breeding ratio is very small, amounting to a reduction of $\sim 1 \%$.

This graphite region should last for the full reactor life of 30 years without replacement. cracking and/or some loss of the segments should not require repair, but it can be easily carried out if necessary.

The use of flat surface segments for a blanket permits the same size and shape blanket modules to be used everywhere in the seactor. It would be difficult to install and replace the large number of different module sizes and shapes if one attempted to follow a curved toroidal blanket surface. The cylindrical modules on each flat surface are close packed on a triangular pitch. There are gaps between the various flat surface segments since they meet at angles as one moves around the toroidal plasma. These gaps are filled with graphite blocks similar to those used in the inner part of the torus so as to prevent neutron streaming and excessive energy leakage through the shield into the magnets.

The flat surface segment design proposed here essentially forms the shield and remains in place during normal replacement ol repair of the blanket modules. However, the segments are 
detachable and can be removed as units, if necessary, by the crane above the reactor. It is not necessary to remove the toroidal magnet coils in order to remove the segments. The segments are designed to be removed through the outer gaps between the TF coils.

The segments contain internal beam members made of aluminum alloy and are supported from 24 bulkheads around the major circumference of the reactor. The bulkheads are affixed on massive pads under the reactor, with a single ring of blanket modules around the circular opening in the bulkhead through which the plasma ring passes.

Most modules (Type B modules) have small diameter stepped shield plugs containing the coolant lines, and those plugs extend through the main shield formed by flat surface segments. A fraction (typically one out of seven) of the modules (Type A modules) have removable shield plugs above them, of diameters somewhat greater than the module. When the Type A module and its shield plug are removed, an access hole is provided through which Type $B$ modules can be removed.

All module piping connections are made outside the shield. When the reactor is shutdown every year for replacement of onethird of the blanket modules, the reactor is first allowed to cool down for $\sim 10$ days so that the $\mathrm{Na}^{24}$ can decay to negligible levels. During the last 3 days of this cool-down period, the piping connections of the moulules to be removed can be disconnected. This can be done without auxiliary shielding for the working crews, since the main shield should be adequate. During the remainder of the 30-day shutdown period, 2500 old blanket modules are removed (the ones that have been in the reactor for 3 years) and new ones inserted. Five work crews working around the clock ( 4 shifts per crew) should be enough to remove and 
replace one Type $A$ module and six adjacent Type B modules. Each module will have ar activity level of about 3 curies, and can be withdrawn into a shielded cask. The low activity per module allows the casks to have relatively minimal shielding and to be much lighter than conventional casks.

Some shadow shielding may be required around the access hole when the adjacent modules are being removed. It should not be necessary to have any personnel go down the access hole into the reactor volume. All connections are outside the shield, and the adjacent Type $B$ modules can be dropped onto a holder beneath them, moved over to the access hole by external contro?, and then withdrawn through the access hole.

The primary vacuum tank seems preferable to having a primary vacuum seal outside the shield with the consequent worry about leaks at the many penetrations caused by coolant lines. The coolant line for each module can be bundled together with a small secondary vaccum container around the point where the module/header connections are made, if desired. These can be quick disconnects to minimize replacement time. 


\section{Plasma Confinement - Same as UWMAK-1}

3. Fuel Injection -- Same as UWMAK-1

4. Plasma Heating - same as UWMAK-1 
5. Mechanical Features of the Very Low Activity Blanket and Shield

\subsection{Overall Assembly}

The reactor is constructed in the form of a torus with an octagonal blanket cross section surrounding the plasma. Figure 5-1 shows the principal parts of the machine: blanket modules on the inside of the corus facing the plasma, the shield slabs, the field magnet coils, and the graphite reflector region on the inside of the torus. Not shown are the divertor and transformer coils. These would be similar to those in UWMAK-1 and would not affect the blanket design. Figure 5-1 also shows standard components such as concrete tank-like vacuum container lined witl steel, handling cranes, passageway for removal of modules, containment dome, etc.

The blanket modules convert the bremsstrahlung and neutron energy to a useful form of heat to drive a power plant, and also breed the makeup tritium. The shield sections reduce the neutron leakage to tolerable levels for operation of the superconducting coils and maintenance work.

The blanket and shield regions around the major circumference of the torus have been divided into 24 equal sectors, each of which is supported on both ends by a bulkhead which extends upward from the floor. See the photograph of the reactor model in Figure 5-2. The field magnets are supported by massive saddles at the bottom.

Each of the 24 sectors is formed from 8 flat shield slabs. These slabs are normally left in place during the 30-year life of the reactor, but can be detached and removed individually, if necessary, using the crane inside the primary vacuum tank. slabs would only be removed if non-repairable damage occurred to them, or if their associated blanket modules could not be detached and new ones replaced.

The bulkheads connect to and support the adjacent flat segment shield slabs. Each bulthead has a single row of blanket modules 
around the plasma, with a shield region outside the blanket modules. The blanket modules are detachable and can be removed through access holes in the adjacent flat segment shield slabs.

\subsection{Module Design}

A typical low activity blanket module is shown in Figure 5-3. An outer cylindrical shell of aluminum is cooled by water flowing in passages in the shell wall. The aluminum wall thickness is fixed by the pressure of the helium coolant which flows through the interior of the module, the module diameter, and the allowable maximum stress in the aluminum.

The dished head of the aluminum shell is called the first wall, and receives bremsstrahlung radiation from the plasma. The Al head can be coated with a low material (e.g., Be) to reduce impurity effects in the plasma. A graphite curtain can also be used between the Al heads and the plasma, if desired.

The fast neutrons from the DT reactions in the plasma pass through the first wall into the interior of the module. A neutron multiplication region is placed directly behind the dished head of the module, with $(n, 2 n)$ reactions in beryllium generating sufficient new neutrons to compensate for neutrons lost by parasitic reactions with module materials.

This neutron multiplication region is formed by filling the disined head of the module with beryllium and LiAl balls. The 1centimeter diameter Be balls are formed by hot pressing Be powder, while the LiAi balls are formed by pressing LiAl pellets of several hundred micron diameter into balls of 1 centimeter diameter with inert filler material. Sufficient porosity is retailed that tritium leaving the surface of the LiAi pellets can quickly escape into the helium coolant.

A neutron moderating region is placed directly behind the neutron multiplying region. This moderating region is composed of 
graphite rods and small $\mathrm{LiAlO}_{2}$ pellets. It is divided into inner and outer moderating regions. The principal distinction between the two regions is that the graphite in the inner moderating zone is replaced because of neutron damage, while the graphite in the outer zone may not have to be replaced.

The graphite is in the form of square rods, 1 inch on a side, with helium coolant passing up between the rods. Small diameter 1 ithium aluminate particles ( $20 \mu$ diameter) for tritium breeding are mixed with graphite powder and loosely compacted into pellets which are inserted into hollow graphite rods. After reaching the surface of the $\mathrm{LiAlO}_{2}$ particles, the bred tritium readily escapes through the pores of the pellets and through a number of holes in the walls of the hollow graphite rods.

The water coolant passages in the aluminum shell are $0.75 \mathrm{~cm}$ in diameter, and are spaced one and one-half diameters apart. The inlet channels, connected in parallel, go down one-half of the circumference of the module, across the dished head, and up through the other half of the module circumference. The water channels can be formed either by a roll bonding process in which two layers of aluminum are rolled together, with the passages formed by expanding the shell with gas pressure, or by brazing an inner and outer shell together with silicon braze. Both methods are straightforward and irexpensive.

In previous studies of very low activity blankets, a form of aluminum known as sintered Aluminum Product (SAP) was used. SAP consists of pure aluminum strengthened by a dispersoid of micronsized aluminum oxide particles (typically 10\% by weight). It retains high strength (typically 10,000 psi) at high temperatures (e.g., $\left.400^{\circ} \mathrm{C}\right)$, but has relatively limited ductility (e.g., 1\%). Fabrication technology is more difficult than that for conventional wrought alloys, but should become easier with further development work. 
For this study it has been decided to use a more conventional form of aluminum, a wrought Al-Mg-Si alloy similar to those in the 6000 series. These alloys are strong, ductile, and among the easiest to work with of the various aluminum alloys. They include some small amounts of objectionable elements like $\mathrm{Cr}$ and $\mathrm{Mn}$, which are added for such purposes as grain size control. They do not directly affect alloy strength, particularly at elevated temperatures. It seems likely that a satisfactory Al-Mg-Si alloy can be developed without objectionable additives, using non-activating elements to control grain size (perhaps vanadium or boron). Development and characterization of such an alloy will require several years, however (1).

The wrought alloys of aluminum have substantially lower temperature capabilities than SAP, however. For purposes of this study, we have assumed that the non-activating Al-Mg-Si alloy that would be developed, will have properties similar to 6061 . The yield strength is taken as $14,000 \mathrm{psi}$ at $400^{\circ} \mathrm{F}$ over the 3-year life of the blanket modules.

With proper alloying additions, aluminum exhibits low corrosion rates in water up to temperatures of about $600^{\circ} F$ (2). Unfortunately, these alloying additions (copper, nickel, etc.) would cause excessive neutron activation. It is assumed for this study that non-objectionable alloying additives can be found which will protect the Al-Mg-Si alloy at $400^{\circ} \mathrm{F}$. The water flow velocity is low enough, approximately two feet per second, that protective films on the coolant passages will probably not be stripped off. With reduction of the energy fraction going to the cool aluminum zone, the temperature of the aluminum shell can be lower without affecting the overall cycle efficiency. Some relatively small modifications should reduce the cold energy fraction to $15 \%$ from the present $32 \%$, which should permit lowering the aluminum 
temperature by 100 to $200^{\circ} \mathrm{F}$. In addition, the aluminum could be cooled either by a separate helium coolant circuit, or more probably, a single helium circuit could be used, with the inlet helium removing energy from the aluminum shell and then circulating through the hot interior of the modules. This option will be examinea in a subsequent section.

In the present cesign, a separate helium circuit is used to cool the interior of the module. The inlet heljum passes down through an annular channel formed by the aluminum module shell and a thin inner aluminum liner. The helium inlet temperature matches the aluminum module temperature. After reaching the dished head of the module, the inlet helium stream passes up through a porous aluminum distributor plate just inside the dished head. The helium then passes through the layer of Be and LiAl, through the graphite cloth or aluminum screen that separates the neutron multiplication and moderating regions, and then through the stack of graphite rods that forms the inner and outer moderating regions.

A cylindrical layer of low density alumina ceramic thermally isolates the hot and cold zones. All neutron and gamma energy directly deposited in this insulating layer is assumed to flow into the cool aiuminum zone and heat flow from the hot to coid zone is computed assuming no heat source in the insulator. These assumptions overestimate the energy leaking from the insulator into the cold zone. (Actually, since the maximum internal temperature in the insulator is considerably higher than either the hot or cold zone temperature, only about one-half of the energy deposited in the $\mathrm{Al}_{2} \mathrm{O}_{3}$ insulator flows to the cold zone.) A minor modification will substantially lower the energy going to the cold zone. If part of the helium coolant flow radially through the insulator into the hot zone, and the local flow rate is adjusted to make the temperature of the helium leaving the inner surface of the insulator 
equal to the hot zone temperature, almost all of the heat deposited in the $\mathrm{Al}_{2} \mathrm{O}_{3}$ insulator will end up in the hot zone.

\subsection{Stress Analysis of Module}

Figure 5-4 shows the stress distribution in a module shell, for an internal helium pressure of $300 \mathrm{psia}$ and a water coolant pressure of 750 psia. The analysis was carried out using a 2-dimensional axisymmetric finite elemert computer code with a 2400 element capacity. The mechanical properties of the aluminum shell were taken as the same for 6061 alloy.

The cylindrical module shell and dished head were divided into a large number of elements. For purposes of analysis, the circular water coolant channels were assumed to be equivalent to having an annular gap between the inner and outer layers of the module shell, tied together by aluminum ligaments of the same cross sectional area as those in the actual shell. This assumption has relatively little effect on the final result, since the internal helium pressure has a much larger effect on stress in the module shell than the water pressure does.

The stresses shown in Figure 5-4 are maximum primary stresses in the inner and outer layers of the module shell. Stresses in the aluminum ligaments that tie the two layers together (i.e., the aluminum between the water coolant channels) are not shown in the plots. These stresses are comparable to those shown for the inner and outer layers, however.

The module shell was assumed as isothermal at $400^{\circ} \mathrm{F}$. There will be small thermal gradients, however, due to the $\Delta T$ across the water cooling circuit, and the temperature differences necessary to drive the energy deposited by bremsstrahlung and neutrons. The thermal stresses will be largest in the outer layer of the dished head where the bremsstrahlung radiation is received. Here they are about 800 psi and will not significantly limit coolant pressures, since 
the stresses caused by the helium and water coolant pressures are much greater. One of the advantages of aluminum over structural materials like stainless steel is the much smaller thermal stress levels achievable with aluminum. Since the Tokamak will operate in a pulsed mode, tens of thousands of accumulated cycles will be experienced by any first wall during the life of the blanket. The resultant cyclic thermal stresses may cause severe fatigue problems if they are not small. With aluminum as the first wall material, fatigue effects due to cyclic thermal stresses should be relatively small.

The maximum stress experienced by the aluminum shell is calculated to be 7000 psi or about $50 \%$ of the yield strength. This is probably sufficient safety factor considering the elevated operating temporature. If a larger safety factor is desired, it can be achieved by either operating the aluminum shell at a lower temperature, decreasing module diameter, increasing module wall thickness, or decreasing the helium coolant pressure, or by some combination of the above. If design modifications to reduce the energy fraction going to the cool aluminum zone are made, then the optimum course is probably to reduce the module wall temperature.

5.4 Module Damage From Radiation and Sputtering

There is no data to indicate how serious the long-term neutron damage effects will be for aluminum alloys in fusion reactors. The total displacements per atom will be comparable to those in fission reactors, for the same fluences, but the production of $\mathrm{H}$ and He atoms by $(n, p),(n, d),(n, \alpha)$, etc reactions will be much greater in fusion reactors. The helium production appears to be the most troublesome effect, since it cannot diffuse out of the aluminum alloy, and will probably collect at various dislocation sites, causing embrittlement. Total helium concentration in the first wall after 3 years of operation will be $\sim 1000 \mathrm{ppm}$. The helium embrittlement will 
probably result in higher strength and lower ductility for the a.luminum. Displacement damage is not expected to be as serious a protlem. The fast neutron fluence $(E>0.1 \mathrm{MeV})$ is about $2 \times 10^{22}$ $\mathrm{n} / \mathrm{cm}^{2}$ after 3 years operation (80\% load factor).

Damage to other blanket components will occur, but these are only filler materials inside the aluminim module shell, and cracking or swelling should not sause any serious problems. The graphite rods will initially shrink, causing a small increase in the helium flow area. This in turn wili slightly lower the heat transfer coefficient and increase the temperature of the graphite rods, but this effect is essentially negligible. The rods will start to expand beyond their original volume at a fluence of $\sim 3 \times 10^{22} \mathrm{n} / \mathrm{cm}^{2}$. but will be replaced by that time. cracking of the graphite rods will have no effect on module performance.

The Be balls will swell because of the neutron induced helium production in them; however, the bed is very shallow (average heigh: diameter ratio is $\sim 1 / 6$, and it should readily expand to compensate for the relatively small amount of swelling, which should be on the order of a few percent.

Sputtering appears to be no particular problem with aluminum modules. With no divertor and a sputiering yield of $10^{-2}$ per plasma ion, only $3 \%$ of the outer layer (i.e.. the layer between the plasma and the water coolant channel) of the aluminum dished head would be sputtered away during the 3-year life of the module. with a $90 \%$ effective divertor, the amount sputtered would be an order of magnitude less. Aluminum has a relatively low $\mathrm{z}$, and the bremsstrahlung enhancement from aluminum impurities in the plasma will be several times less than from a niobium or stainless steel first wall. A woven graphite curtain in front of the structural wall, as proposed by the UWMAK-2 design (3), will result in even lower impurity effects. 


\subsection{Use of a Graphite Reflector Region for the Innex Part of the Toroidal Blanket}

Access to the inner part of the toroidal blanket surface is very difficult, if not impossible. If adequate access space. say l-meter long the major radius, were provided behind the shiela at this point, the maximum magnetic field experienced by the toroidal field coils would be increased by $20 \%$. This would greatly increase the magnet cost, and necessitate either operating the $\mathrm{NbTi}$ superconductor at $-2{ }^{\circ} \mathrm{K}$, or using $\mathrm{Mb}_{3} \mathrm{sn}$ conductor or cryogenic aluminum for part of the coil. This problem is common to all tokamak reactors unless their size is much larger than that now contemplated.

A way out of this problem has been proposed previously $(4)$. and is incorporated in this design. The inner fart of the toroidal blanket surface, in this case the blanket that would be attached to the inner one of the 8 rlat shield slabs forming the octagonal structure shown in Figure $5-I$, is made of a single segmented structure of graphite blocks (typically $15 \mathrm{~cm}$ square and about one meter long) attached to the innermost shield slab. Most of the energy deposited by neutrons and gammas in the graphite structure is thermally radiated back across the plasma (which is virtually transparent) to the rest of the blanket. The graphite surface temperature is $21800^{\circ} \mathrm{C}$ at the wall loading in this design. This is well below the limit of $\sim 2000^{\circ} \mathrm{C}$ set by evaporation (above this temperature, graphite evaporation results in excessive bremsstrahlung losses). The maximum internal graphite temperature is $-2200^{\circ} \mathrm{C}$.

The rear third of the $1 \mathrm{~m}$ thick graphite block structure (near the shield) contains passages for aluminum alloy coolant tubes, and operates at lower temperatures $\left(\mathrm{e} . \mathrm{g} . \sim 1000^{\circ} \mathrm{C}\right.$ ) than the front 
two thirds of the graphite structure. Heat is radiantly transferred from the rear third of the graphite structure to the coolant tubes. Solid lithium compounds are used for tritium breeding in this rear third of the graphite structure. This probably would take the form of LiAlo $_{2}$ pellets inserted into holes in the graphite, or alternatively, LiAl pellets inside the coolant tubes. In the first case, the bred tritium could enter the vacuum region between the first wall and the plasma, and would be recovered with the plasma exhaust. In the second case, the bred tritium would be recovered from the helium coolant in the tubes, in a manner similar to that from the main helium stream.

The front two thirds of the graphite structure operates at a high enough temperature that radiation damage effects should anneal out. The rear third operates at too low a temperature for annealing, but the fluences in this region are low enough to permit operation for 30 years without replacement (similarly, the coolant tubes should not need replacement). Cracking or dimensional changes in this graphite reflector region should not normally require removal of the graphite, and it is expected that only a small amount of graphite will have to be replaced during the life of the reactor. Stresses in the graphite are expected to be very low, and cracks will not resuit in coolant leaks to the plasma. If it is necessary, replacement of damaged graphite blocks could be accomplished by a simple pullout of the old block and a simple push-in of the new one.

The graphite reflector region comprises only about $5 \%$ of the total blanket area and will have a negligible effect on overall breeding ratio (about a $1 \%$ reduction). Overall net thermal efficiency will be slightly reduced, for example, from $32 \%$ to $31.75 \%$. 


\subsection{Shapes and Weights of Blanket Shield Components}

Figure 5-5 is a drawing again showing the octagonal shape of the torus. It also contains a table of weights of parts and amounts of various materials required for construction of a low activity blarket and shield.

\subsection{Maintenance Operations}

The principal maintenance operation considered in this preliminary design is removal and replacement of modules. No definitive information is available concerning the effect of radiation on the aluminum module. This preliminary design assumes the life of a module to be three years, and replaces one third of the modules each year. Some parts may be reconditionable and reusable.

There are two cases to consider: (a) removal and replacement of an occasional failed module: and (b) annual replacement of one third of the blanket, some 2000 to 2500 modules.

Reference to Figure 5-6 will indicate the plan which has been developed. Two modules are shown, one on the left labeled, "ACCESS BLANKET MODULE" and one on the right labeled, "BLANKET MODULE." Also shown are shield plugs and an indication of the extensive water and helium cooling manifolds.

some suitable small fraction (about 1 in 7 , depending on further study) of the modules will be access modules. These are termed Type A modules. In order to replace a damaged module, or a number of modules scheduled for replacement, the nearest Type A module is located, the piping disconnected, the shield plugs pulled out, and the module pulled out. The shield plug weigh about $2500 \mathrm{lb}$., the module about $1100 \mathrm{lb}$. If the Type $\mathrm{A}$ module is not to be replaced, it is simply put aside and returned after the other modules have been replaced. If it must be 
replaced, it is sent first to removed module storage and then to the reprocessing plant as described in section 11. To remove blanket modules adjacent (Type $B$ modules) to the Type A module. the piping must be disconnected; a tool then is lowered through the Type A module opening. This tool seizes and holds the Type B module, which is then lowered towards the plasma axis. Another tool then grasps the Type B blanket module and draws it out through the access opening. After this, it can te sent to removal storage and reprocessed, as described in section 11.

Both Type A and B modules are supported by ring type supports on the inner part of the shield in a low flux zone. They can be detacined from these supports by a simple rotation and pul1-out, once the piping connections outside the shield have heen broken. As has been noted, the Type A modules can be pulled directly out through the shield, once their shield plug is removed, while the Type B modules must first be lowered towards the plasma centerline, moved laterally to the nearest access hole, and then pulled out through the access hole.

For the case of replacement of several damaged modules, the reactor downtime will be on che order of 1 week. It will require several days for the $\mathrm{Na}^{24}$ activity to cool down to the point where work crews can gain access to the external shield area, and one to two days to remove the damaged modules. Local shielding will be needed when the access hole is opened and the damaged modules withdrawn.

One-month downtime is estimated for the scheduled yearly replacement of one third of the blanket modules. The cool-down time will then be on the order of 10 days. Five work crews, with 4 shifts per crew, should be sufficient to remove the 2500 modules in 20 days. Each shift will have to remove one access module plus 
its six adjacent modules. The cost of such crews, assuming $5 \mathrm{men} / \mathrm{crew}$ and 4 shifts, and $\$ 100 / \mathrm{man}$ shift, is still minimal, on the order of $\$ 300,000$. This is much less than the interest on capital investment (which will be on the order of $\$ 8$ million) for the same period, and considerable savings could be expected if shorter periods are used. We cannot go into this question in more detail, but it appears that a shorter period should be more optimum, and in fact, it may pay to replace the whole blanket at once. In addition, a higher first wall load may be more optimum with shorter blanket lifetimes, since reactor costs in $\$ / \mathrm{kw}$ should be smaller.

It would appear that the aluminum modules described in this design would be capable of at least 3 times the wall loading assumed here, that is, they could be used at $5 \mathrm{MW}(\mathrm{th}) / \mathrm{m}^{2}$. The pressure drop of the water and He coolants through the blanket modules is very low, and flow velocities of 3 times the present design values would cause no problem. 


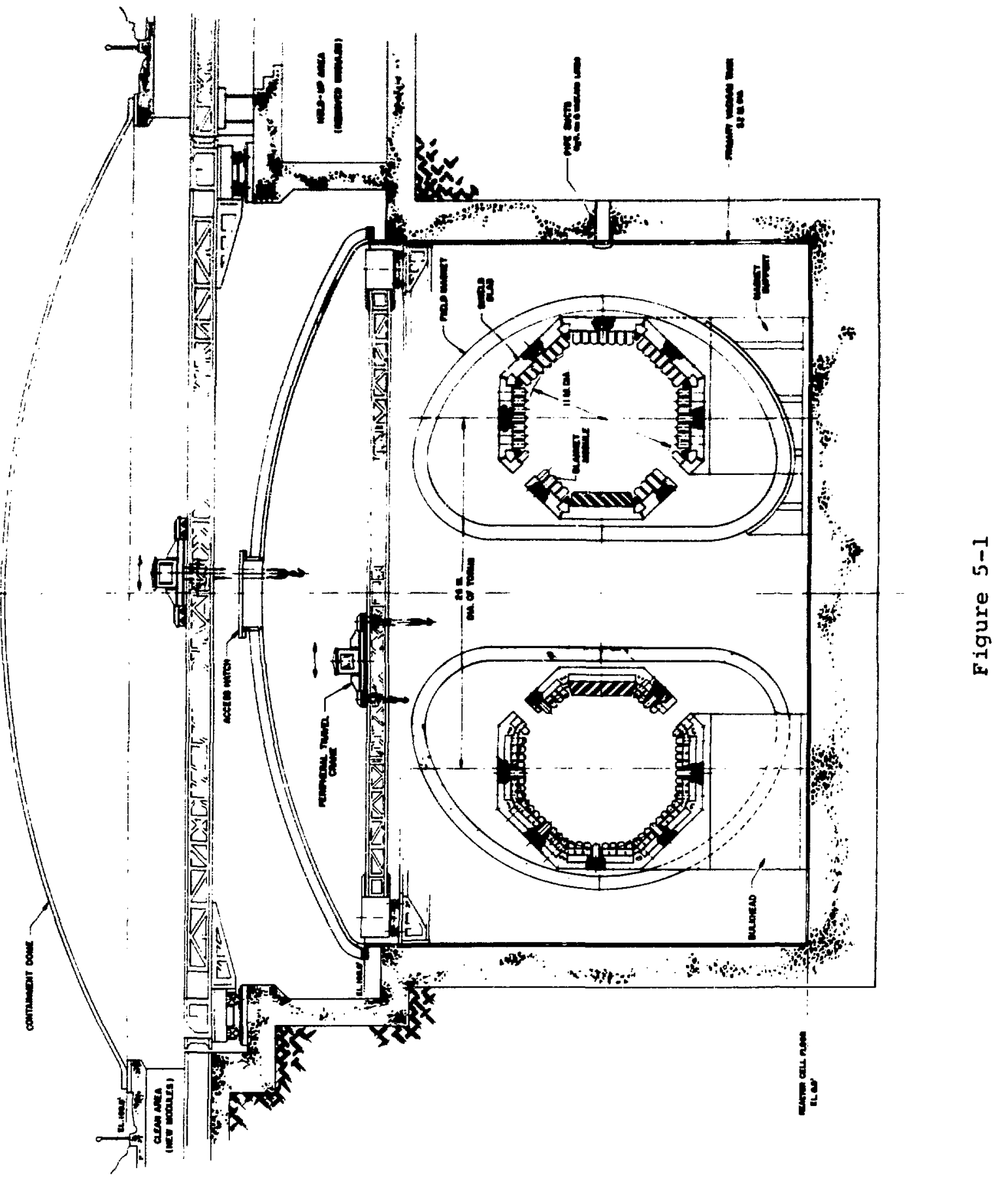




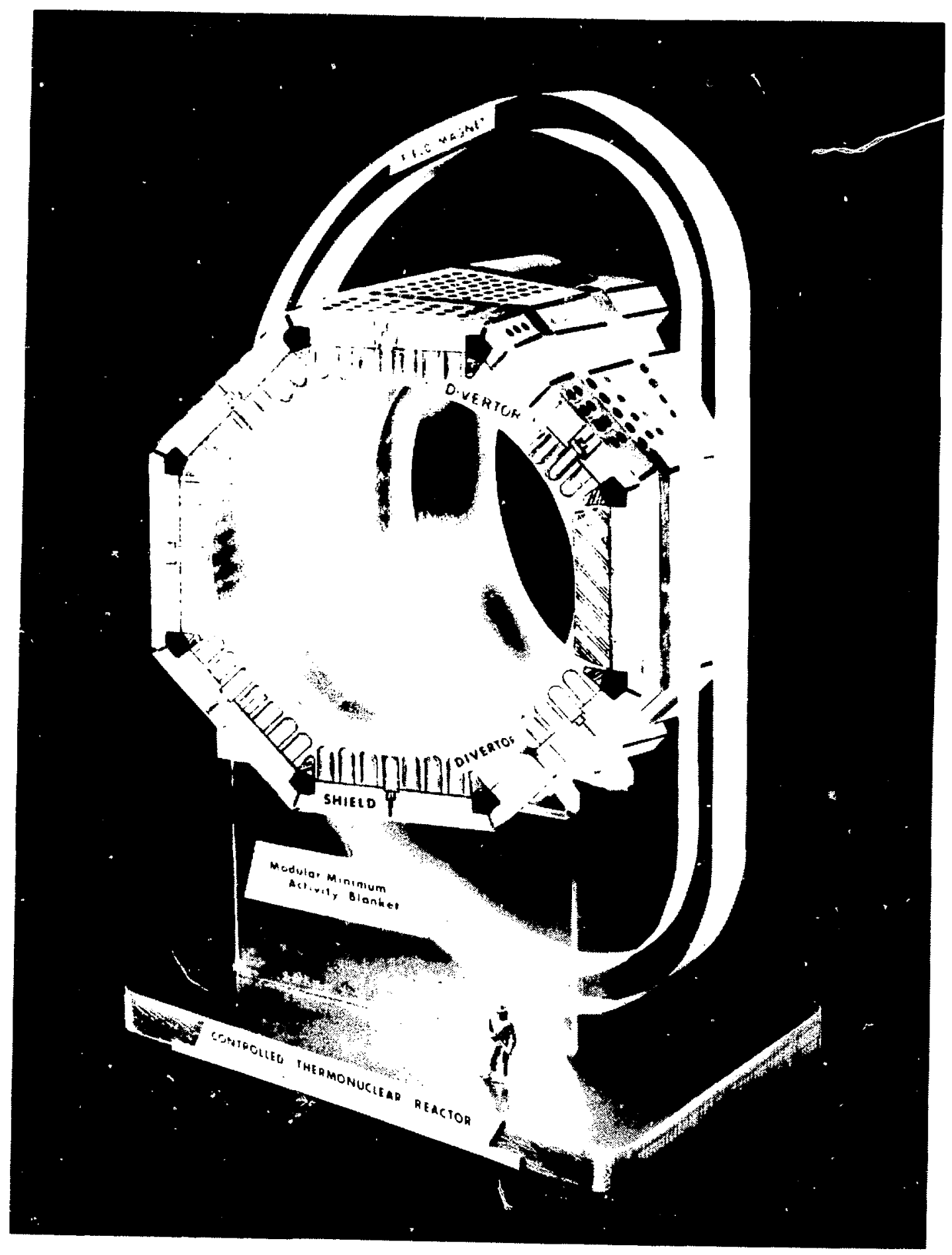

Figure 5-2 


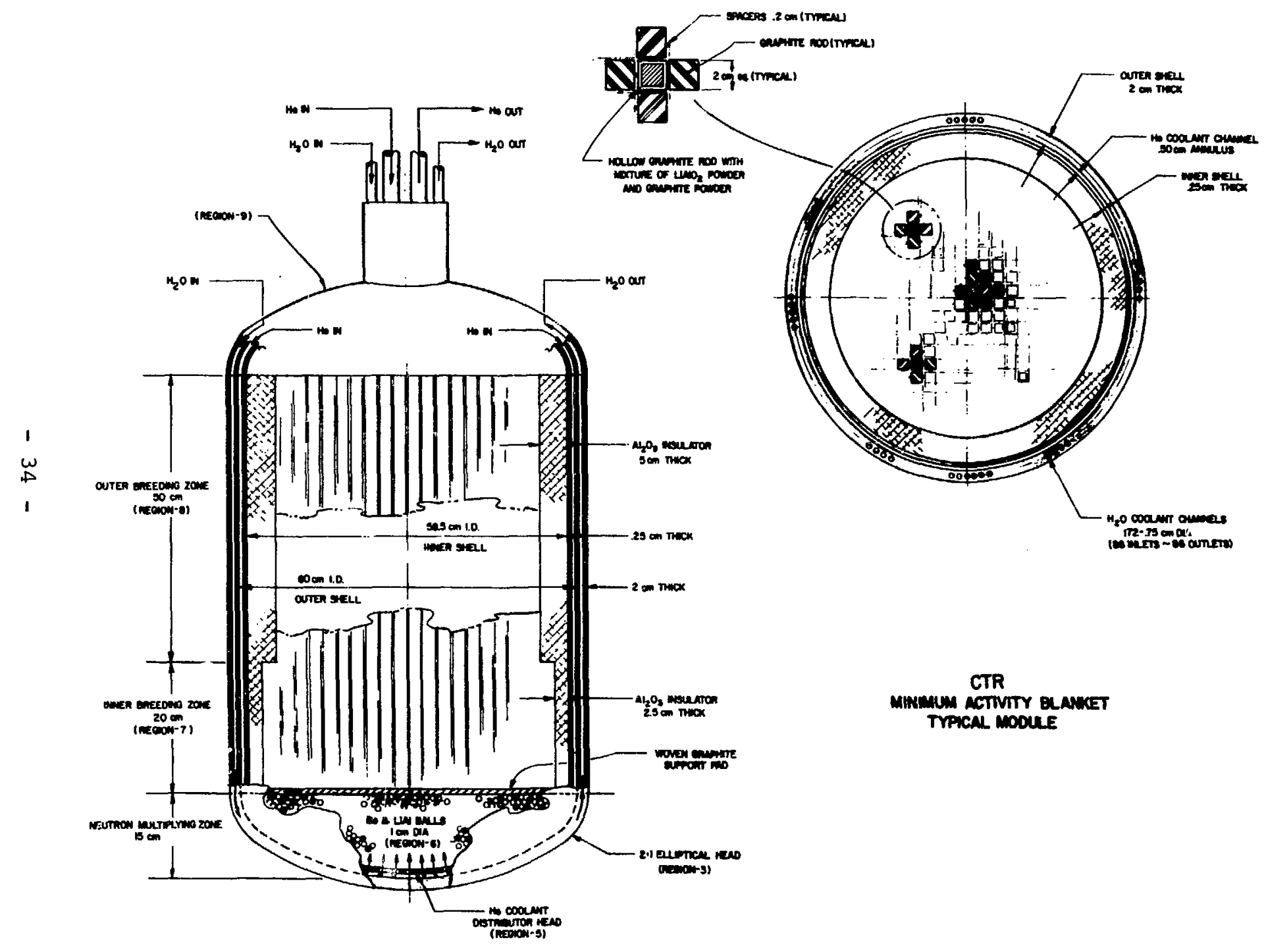

Figure 5-3 


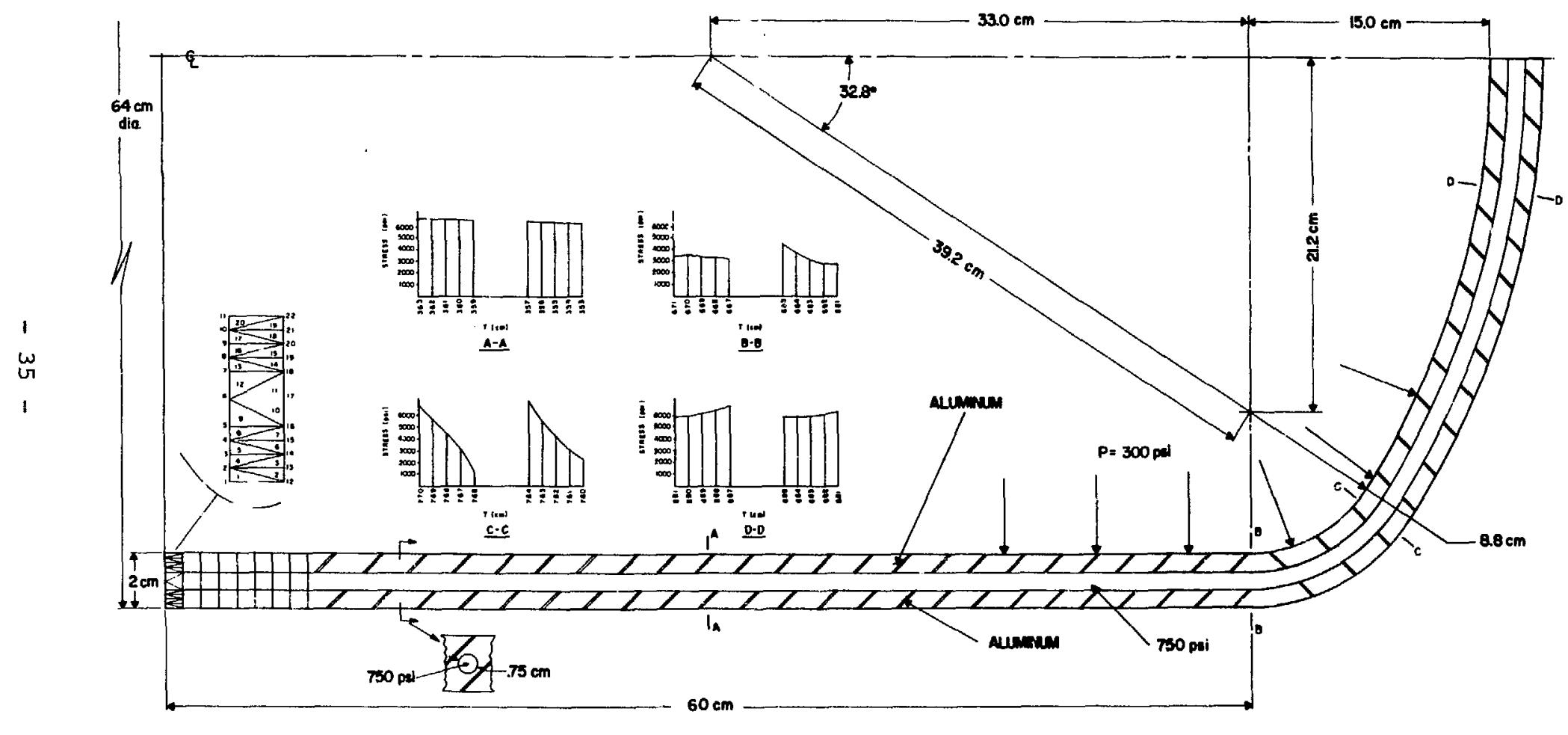

STRESS DISTRIBUTION IN MODULE SHELL

Figure 5-4 


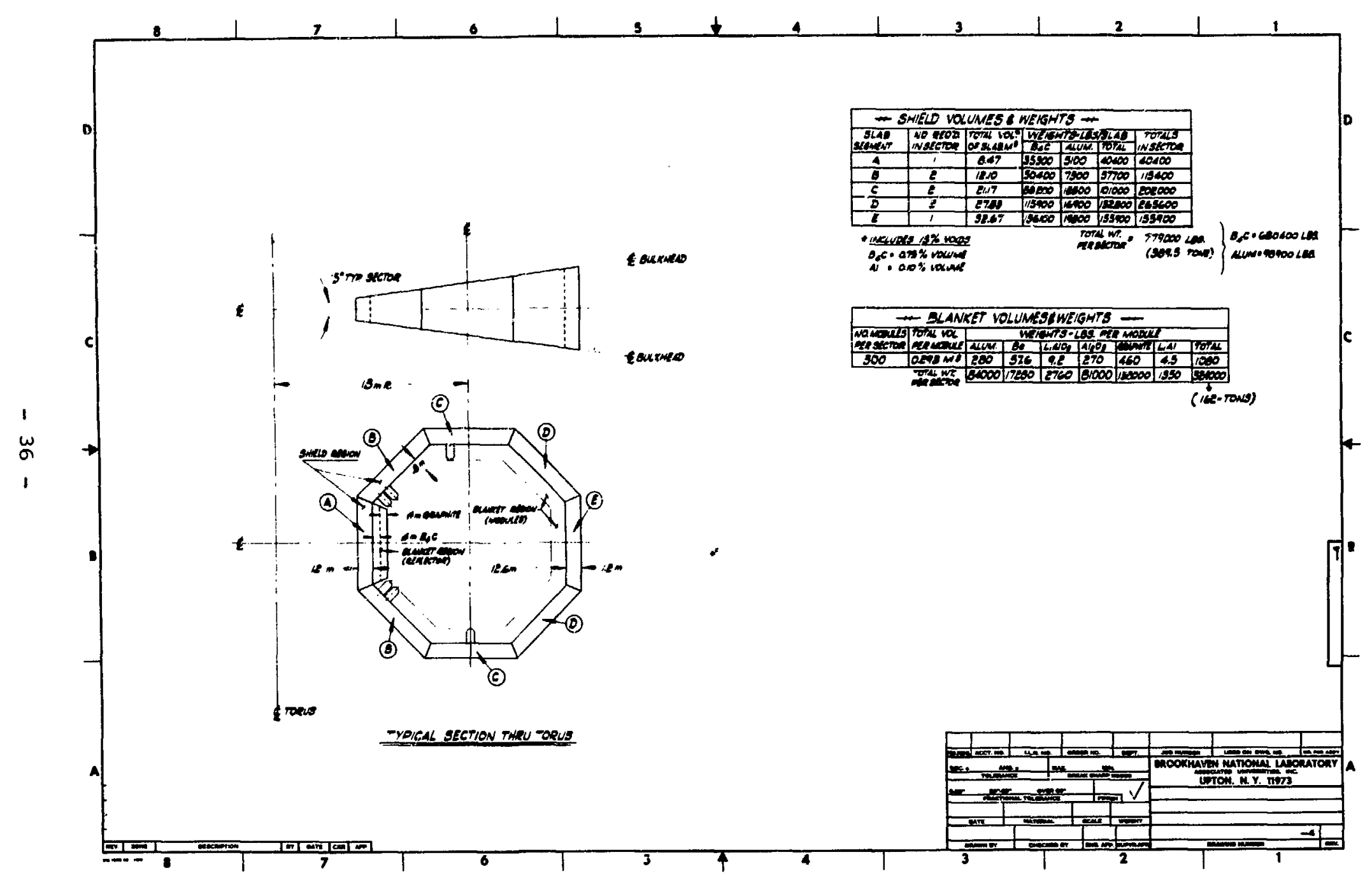

Figure 5-5 


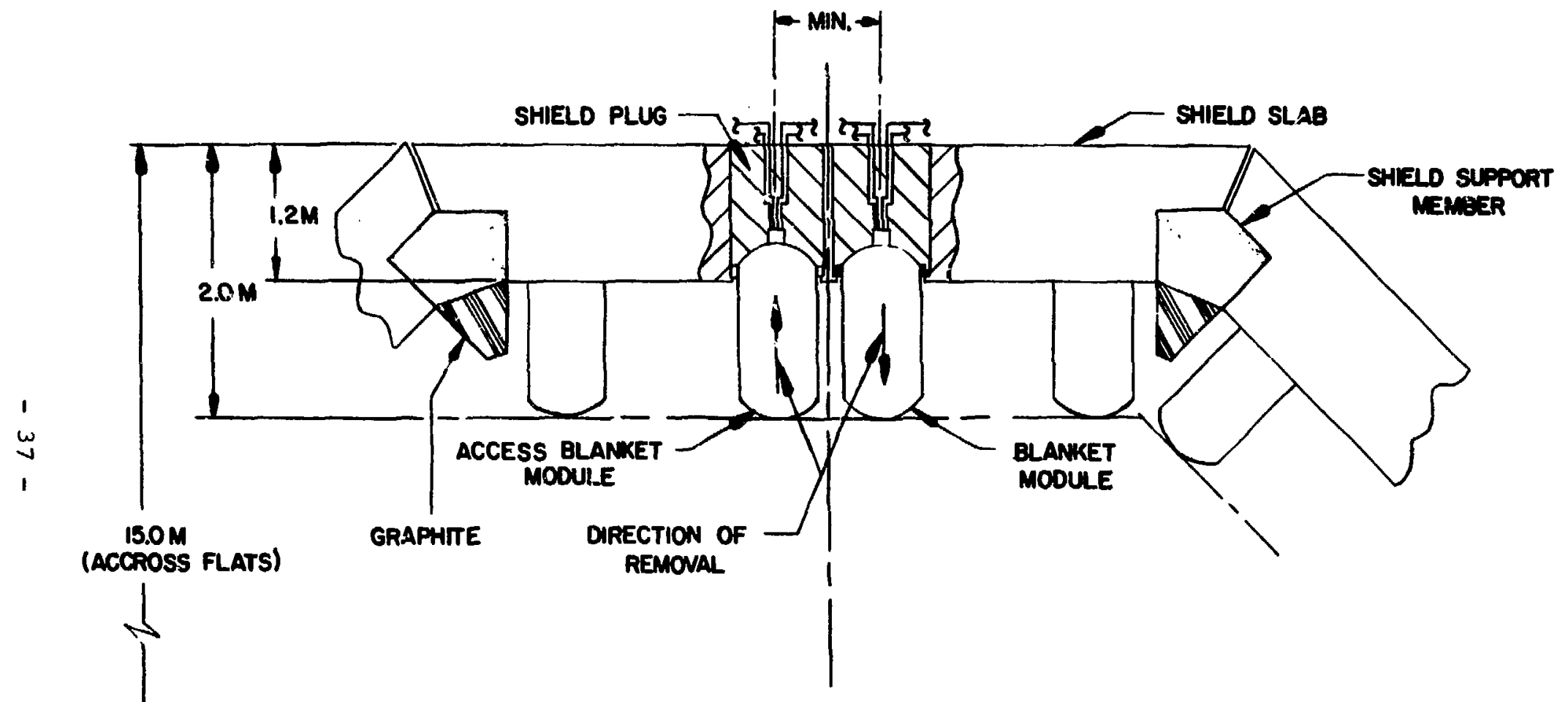

TYPICAL ARRANGEMENT OF BLANKET

MODULES IN SHIELD SLABS

Figure 5-6 


\section{Neutron Activation and Breeding}

The blanket module is displayed in Figure 5-3. Each zone in the module is homogenized for the one-dimensional ANISN calculations. (see Figure 6-1) The compositions, volume fractions, dimensions, and homogenized atomic densities for each zone are given in Table 6-1. The impurity concentrations which were included are listed in Table 6-2.

The cross sections used in the ANISN calculations are based on ENDF/B-III (1). The cross sections were pracessed into a multigroup set at Oak Ridge National Laboratory using the SUPERTOG program (2) and distributed through the Radiation Shielding Information Center as RSIC Data Library DLC-2/100g (3). This set represents up to a $\mathrm{P}_{8}$ approximation to elastic scattering angular distribution, and a 100-group structure with energy boundaries identical to those in the GAM-II library (4). Only down scattering is allowed, and group 100 is a thermal group with cross section values based on a Maxwellian average at $0.0253 \mathrm{eV}$. Retrieval programs were used to generate a $P_{3}$ cross section library tape for the ANISN program (5) from these cross section sets.

The neutron flux distributions are calculated using the ANISN program. The geometry is represented as an infinite cylinder with a vacuum boundary condition at the outer radius. A $\mathrm{P}_{3}$ option is used for the order of anisotropic scattering, and an $\mathrm{s}_{8}$ option for the angular quadrature. The $14.1 \mathrm{MeV}$ source neutrons are taken to have a uniform spatial distribution in the plasma region. In the GAM-II group structure, this energy falls within the koundaries of the highest energy group (13.5-14.918 MeV). For the neutronics calculations, the magnitude of the source is normalized to one neutron per linear centimeter along the axis of the cylinder. For the purpose of obtaining reaction rates, the ENDF/B-III data tapes are processed to provide group averaged cross sections 
within the GAM-II energy groups structure, for all reactions for which data was available, for each of the nuclides present in the system. A neutron balance is obtained using those cross sections, the approximate nuclide abundance, and the flux distributions computed by the ANISN program. The resulting neutron balance is given in Table 6-3.

The breeding ratio was found to be 1.2 tritons produced per fusion neutron.

The energy escaping from the shield is several ev per fusion. For $14 \mathrm{MeV}$ neutrons, the ratio of the flux at the outside edge of the shield to the flux at the inside edge of the first wall is $\sim 10^{-7}$. Consequently the leakage energy is small enough that its input to the superconducting magnets will be tolerable. The total and $14 \mathrm{MeV}$ neutron flux are shown in Figure 6-2. The flux shown corresponds to $5000 \mathrm{MW}(\mathrm{th})$ total fusion power (inciuding blanket multiplication).

The burn-up rates (in per cent of the original material for a one-year period) are:

$$
\begin{array}{lr}
\mathrm{Li}^{6} & 10.13 \\
\mathrm{Be} & .16
\end{array}
$$

Thus, after 30 years of operation, $4.9 \%$ of the Be will be destroyed. In this activation analysis, only those nuclides with a halflife of more than 12 hours were incl uded. The activations were calculated using cross sections obtained from ENDF/B-III, where available. Other cross sections were obtained from the National Neutron Cross Section Center, Brookhaven National Laboratory. These cross sections were calculated by the nuclear model computer program THRESH, (6) and are: $\mathrm{Fe}^{54}, \mathrm{Fe}^{56}, \mathrm{Mn}^{55}, \mathrm{Ni}^{58}, \mathrm{Ni}^{60}, \mathrm{sn}^{118}, \mathrm{sn}^{120}$, $\mathrm{Ti}^{48}, \mathrm{zn}^{64}, \mathrm{zn}^{66}, \mathrm{zr}^{90}$, and $\mathrm{zr}^{96}$.

In addition, the cross sections for the two reactions $c^{13}$ $(n, \gamma) c^{14}$ and $o^{17}(n, \alpha) c^{14}$ were approximated by using the thermal 
cross section and assuming a $1 / v$ dependence of the cross section on the velocity. The cross section for $\mathrm{Mg}^{25}(n, d) \mathrm{Na}^{24}$ was assumed to be $100 \mathrm{mb}$ for all energy groups. The cross sections for $\mathrm{Mg}^{24}$ $(\mathrm{n}, \mathrm{p}) \mathrm{Na}^{24}$ were approximatied by those for natural $\mathrm{Mg}$. The major contributor 3 to the activation are listed in Table 6-4 and a graph of the activity as a function of time after reactor shutdown is shown in Figure 6-3.

The heating calculations were broken up into three farts: bremsstrahlung, neutron, and gamma heating. All the bremsstrahlung energy was assumed to be absorbed by the first wall. The neutron heating was calculated using the KERMA factors contained in MACKIB (7), a data tape containing the values of neutron kinetic energy absorbed in materials. The gamma heating was calculated by assuming that the gamma energy absorbed per unit length has an exponeniial dependence on distance into the material (8). Specifically, the derivation of absorbed gamma ener $Y, E_{G^{\prime}}$ with respect to length, , is:

$$
\frac{d E_{G}}{d}=A e^{-\mu} \text {, where } A \text { is a normalization constant }
$$

and

$$
\begin{aligned}
& \mu=.03 \mathrm{\rho} \text {, where } \rho \text { is the density of the absorbing } \\
& \text { material. }
\end{aligned}
$$

The results of these calculations are listed in Table 6-5. 
Table 6-1

BLANKET COMPOSITIONS

Thickness (cm)

Compositions and Density Material

(Volume Fraction)

1. Al $6061(2.75 \mathrm{~g} / \mathrm{cc})$

2. $\mathrm{H}_{2} \mathrm{O}(.8 \mathrm{~g} / \mathrm{cc})$

3. $\mathrm{Be}(1.7 \mathrm{~g} / \mathrm{cc})$

4. LiAl (1.545 $\mathrm{g} / \mathrm{cc})$

5. $\mathrm{Al}_{2} \mathrm{O}_{3}(4.0 \mathrm{~g} / \mathrm{cc})$

6. $G(1.9 \mathrm{~g} / \mathrm{sc})$

7. $\mathrm{LiAlO}_{2}(2.5 \mathrm{~g} / \mathrm{cc})$

8. $\mathrm{B}_{4} \mathrm{C}(2.52 \mathrm{~g} / \mathrm{cc})$

I

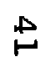

Atomic Densities
(x $10^{22}$ atms/Cc)
1. $\mathrm{Mg}$
2. $\mathrm{Li}^{6}$
3. $\mathrm{Li}^{7}$
4. $\mathrm{H}^{2}$
5. $\mathrm{O}$
6. $\mathrm{Al}$
7. $\mathrm{H}^{1}$
8. $\mathrm{Si}$
9. $\mathrm{Be}$
10. $\mathrm{B}$
11. $\mathrm{B}^{11}$
12. $\mathrm{C}$

\begin{tabular}{|c|c|c|c|c|c|c|}
\hline 3 & 5 & 6 & 7 & 8 & 9 & 10 \\
\hline 2.46 & .31 & 8.00 & 20.00 & 50.00 & 10.00 & 110.00 \\
\hline
\end{tabular}

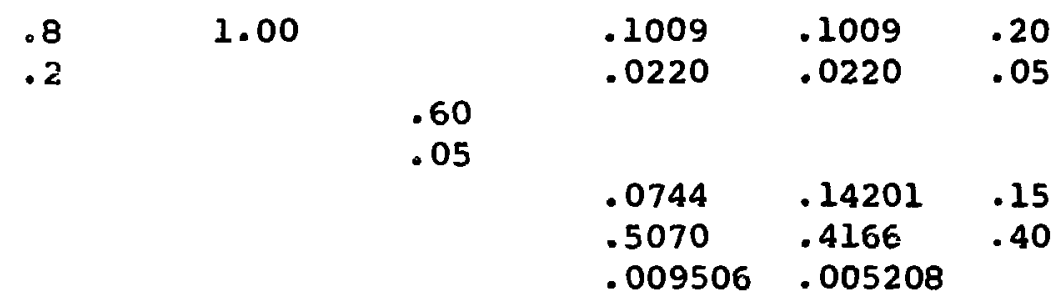

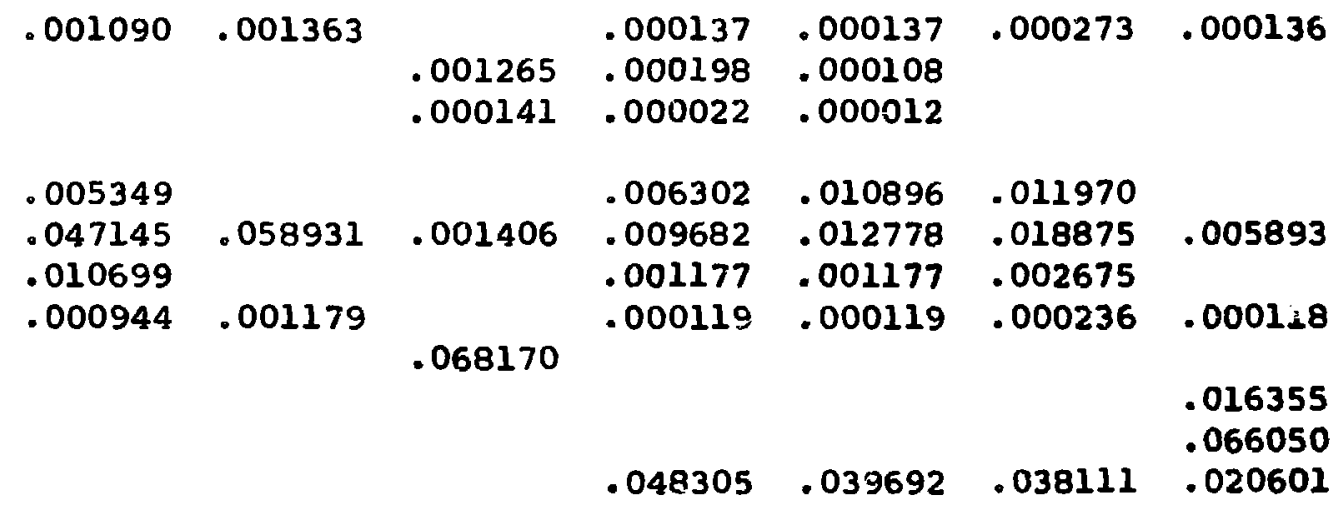

Note: The Li in LiAl and $\mathrm{LiAlO}_{2}$ is $90 \mathrm{a} / 0 \mathrm{Li}^{6}$ and $10 \mathrm{a} / 0 \mathrm{Li}^{7}$ Al 6061 is $96 \mathrm{w} / 0 \mathrm{Al}+2 \mathrm{w} / 0 \mathrm{Mg}+2 \mathrm{w} / 0 \mathrm{si}$ 


\section{Table 6-2 \\ IMPURITY CONCENTRATIONS}

\begin{tabular}{|c|c|c|}
\hline Material & Impurities & $\begin{array}{l}\text { Concentrations of Impurities } \\
\text { (ppm, weight) }\end{array}$ \\
\hline \multirow[t]{6}{*}{ Al } & $\mathrm{Fe}$ & 1. \\
\hline & Mn & 1. \\
\hline & sn & 1. \\
\hline & $\mathbf{T i}$ & 2. \\
\hline & $\mathrm{Zn}$ & 1. \\
\hline & $\mathrm{Zr}$ & 4. \\
\hline \multirow[t]{5}{*}{ Graphite } & $\mathrm{Fe}$ & .5 \\
\hline & Mn & .01 \\
\hline & $\mathrm{Ti}$ & .2 \\
\hline & $\mathrm{Zn}$ & .01 \\
\hline & $\mathbf{Z r}$ & .01 \\
\hline \multirow[t]{7}{*}{$\mathrm{Be}$} & $\mathrm{Fe}$ & 2. \\
\hline & $\mathrm{Mn}$ & 2. \\
\hline & $\mathrm{Ni}$ & 1. \\
\hline & $\mathbf{T i}$ & 2. \\
\hline & sn & 3. \\
\hline & $\mathrm{Zn}$ & 2. \\
\hline & $\mathrm{Zr}$ & 1. \\
\hline
\end{tabular}


Table 6-3

NEUTRON BALANCE

Nuclide and

Reaction

$L i^{6}(n, 2 n)$

abs

$(n, T)$

$\mathrm{Li}^{7}(\mathrm{n}, 2 \mathrm{n})$

abs

$$
o^{16} \begin{array}{r}
(n, T) \\
(n, y) \\
(n, p) \\
(n, d) \\
(n, \alpha)
\end{array}
$$

$\mathrm{Al}^{27}(n, 2 n)$

$(n, y)$

$(n, p)$

$(n, a)$

$(\mathrm{n}, \mathrm{T})$

$1(n, \alpha)$

$\mathrm{H}^{1}(\mathrm{n}, \mathrm{y})$

si $(n, 2 n)$

$(n, n) p$

$(n, y)$

$(\mathrm{n}, \mathrm{p})$

$(n, d)$

$(n, a)$

\begin{tabular}{ccccccc}
\multicolumn{8}{c}{ Region } \\
\hline 3 & 5 & 6 & 7 & 8 & 9 & 10
\end{tabular}

.00000

.00098

.00034

.00359

.00910

00106

$.00071 \quad .00009 \quad .00001$

$.00022 \quad .00005 \quad .00002$

$.62200 \quad .31260 \quad .24960$

$.00006 \quad .00001 \quad .00000$

$.00001 \quad .00000 \quad .00000$

$\begin{array}{lll}.00057 & .00009 \quad .00002\end{array}$

$\begin{array}{llll}.00000 & .00000 \quad .00000 \quad .00000\end{array}$

$.00159 \quad 00073 \quad .00001$

$.00052 .00023 \quad .00000$

$.00820 \quad .00468 \quad .00009$

.01222

.00124

.00040

$.00198 \quad .00059 \quad .00001 \quad .00000$

$\begin{array}{lllll}.00033 & .00540 & .00845 & .00091 & .00000\end{array}$

.0206

.00267

.0012

$\begin{array}{llll}.00831 & .00359 & .00009 & .00002\end{array}$

$.00556 \quad .00066$

.00003

.0002

$.00148 \quad .00050$

.00001 .00000

.00000

.0000

00000

$.00000 \quad .00000$

.02775

.00342

.0014

.00902

.00341

.00007

.00001

.00345

$.00065 \quad .00096 \quad .00018$

$\begin{array}{lllll}.00001 & .00000 \quad .00000 \quad .00000\end{array}$

$\begin{array}{llll}.00002 & .00000 \quad .00000 \quad .00000\end{array}$

$\begin{array}{llll}.00003 & .00003 & .00000 & .00000\end{array}$

$\begin{array}{llll}.00029 & .00009 & .00000 \quad .00000\end{array}$

$.00001 \quad .00000 \quad .00000 \quad .00000$

$\begin{array}{lllll}.00028 & .00009 & .00000 & .00000\end{array}$ 
Table 6-3

NEUTRON BALANCE (Cont'd)

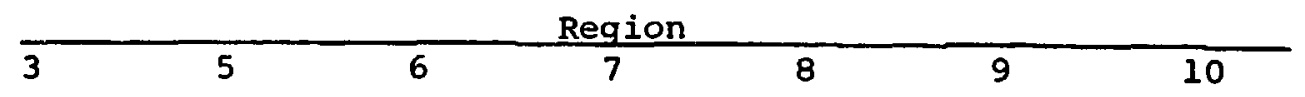

Nuclide and

Reaction

\begin{tabular}{|c|c|c|c|c|c|c|c|}
\hline $\mathrm{Be}$ & $(n, 2 n)$ & & .47170 & & & & \\
\hline & $(n, v)$ & & .00067 & & & & \\
\hline & $(n, P)$ & & .00004 & & & & \\
\hline & $(n, d)$ & & & & & & \\
\hline & $(n, T)$ & & .00890 & & & & \\
\hline & $(n, \alpha)$ & & .03892 & & & & \\
\hline $\mathrm{B}^{10}$ & $(n, d)$ & & & & & & .00003 \\
\hline & $(n, T)$ & & & & & & .00016 \\
\hline & $(n, a)$ & & & & & & .02463 \\
\hline$B^{11}$ & $(n, \gamma)$ & & & & & & .00000 \\
\hline & $(n, p)$ & & & & & & .00000 \\
\hline & $(n, T)$ & & & & & & .00001 \\
\hline & $(n, \alpha)$ & & & & & & .00003 \\
\hline c & $(n, v)$ & & & .00027 & .00033 & .00003 & .00000 \\
\hline & $(n, \alpha)$ & & & .05681 & .01352 & .00018 & .00006 \\
\hline $\mathrm{Mg}$ & .00070 & .00009 & & .00014 & .00004 & .00000 & .00000 \\
\hline
\end{tabular}

Breeding ratio $=1.19$ tritons/fusion neutron. 
Table 6-4

MAJOR CONTRIBUTORS TO ACTIVATION

(Activation in Curies)

Nuclide

$\mathrm{Na}^{24}$

$\mathrm{Mn}^{54}$

$\mathrm{zr}^{89}$

$\mathrm{Sn}^{119}$

$\mathrm{sn}^{117}$

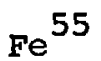

$\mathrm{Zn}^{65}$

$\mathrm{Co}^{58}$

$\mathrm{sc}^{48}$

$\mathrm{Cu}^{64}$

$\mathrm{Zr}^{96}$

$\mathrm{AI}^{26}$
Days After Shutdown

\begin{tabular}{|c|c|c|c|c|c|}
\hline 0 & 1 & 10 & 100 & 1,000 & 10,000 \\
\hline $1.95 \times 10^{9}$ & $6.47 \times 10^{8}$ & $2.94 \times 10^{5}$ & 0 . & 0 . & 0. \\
\hline 7070 & 7050 & 6910. & 5660 & 770. & 0. \\
\hline 6370 & 5160. & 766 & 0 . & 0. & 0. \\
\hline 4740 & 4720 。 & 4610 。 & 3570 . & 280. & 0. \\
\hline 3420 & 3260 。 & 2090 & 24. & 0. & 0. \\
\hline 3350 & 3350 。 & 3330 & 3120 。 & 1660. & 3. \\
\hline 2130. & 2130 & 2070 & 1610. & 124. & 0. \\
\hline 1680. & 1660 。 & 1520 & 634. & 0. & 0. \\
\hline 1670. & 1440 & 37. & 0. & 0 & 0. \\
\hline 1210. & 329. & 0 & 0 . & 0. & 0. \\
\hline 926. & 316. & 833. & 321. & 0. & 0. \\
\hline 785. & 785 & 785. & 785. & 785. & 785. \\
\hline
\end{tabular}




\section{Table 6-5 \\ BLANKET AND SHIELD HEATING}

Listed below are the Bremsstrahlung $\left(E_{B r e m}\right)$, neutron $\left(E_{n}\right)$, gamma $\left(E_{G}\right)$, and total heating $\left(E_{\text {Total }}\right)$ in units of MeV/fusion.

The "cold" volume consises of the aluminum shell, the water, the $\mathrm{Al}_{2} \mathrm{O}_{3}$ and the sides of the distributor plate. The "hot" volume consists of everything else.

Region 4 is the helium cooling gap which does not contribute to the heating.

\begin{tabular}{|c|c|c|c|c|c|c|}
\hline Region \# & \multicolumn{2}{|c|}{ Region Name } & \multirow{2}{*}{$\begin{array}{l}\mathrm{E}_{\text {Brem }} \\
0 . \\
2.55 *\end{array}$} & \multirow{2}{*}{$\frac{E_{n}}{0 .}$} & \multirow{2}{*}{$\frac{{ }_{\mathrm{E}_{\mathrm{G}}}}{0.21}$} & \multirow{2}{*}{$\begin{array}{l}\mathrm{E}_{\text {Total }} \\
0 . \\
4.27\end{array}$} \\
\hline 3 & $\begin{aligned} \text { First wall } & - \text { ho } \\
& - \text { co }\end{aligned}$ & & & & & \\
\hline 5 & Distributor Pla & $\begin{aligned} \text { te } & - \text { hot } \\
& - \text { cold }\end{aligned}$ & $\begin{array}{l}0 . \\
0\end{array}$ & 0.1 .2 & $0^{.03}$ & .15 \\
\hline 6 & $\begin{aligned} \text { Beryllium } & - \text { hot } \\
& - \text { col }\end{aligned}$ & & $\begin{array}{l}0 . \\
0\end{array}$ & $\begin{array}{l}5.94 \\
0 .\end{array}$ & 0.65 & $\begin{array}{l}6.59 \\
0 .\end{array}$ \\
\hline 7 & Inner Graphite & $\begin{array}{l}\text { - hot } \\
\text { - cold }\end{array}$ & $\begin{array}{l}0 . \\
0 .\end{array}$ & $\begin{array}{r}3.08 \\
.67\end{array}$ & $\begin{array}{l}.75 \\
.44\end{array}$ & $\begin{array}{l}3.83 \\
1.11\end{array}$ \\
\hline 8 & Outer Graphite & $\begin{array}{l}- \text { hot } \\
-\operatorname{cold}\end{array}$ & $\begin{array}{l}0 . \\
0\end{array}$ & $\begin{array}{r}1.52 \\
.33\end{array}$ & $\begin{array}{l}.72 \\
.54\end{array}$ & $\begin{array}{r}2.24 \\
.87\end{array}$ \\
\hline 9 & Transition Zone & $\begin{array}{l}- \text { hot } \\
-\operatorname{cold}\end{array}$ & $\begin{array}{l}0 . \\
0 .\end{array}$ & $\begin{array}{l}.01 \\
.01\end{array}$ & $\begin{array}{l}0 . \\
0 .\end{array}$ & $\begin{array}{l}.01 \\
.01\end{array}$ \\
\hline 10 & $\begin{aligned} \text { shield } & \text { - hot } \\
& - \text { cold }\end{aligned}$ & & $\begin{array}{l}0 . \\
0 . \\
\end{array}$ & .09 & $\begin{array}{l}0 . \\
0 .\end{array}$ & .09 \\
\hline & Total & & 2.55 & 13.28 & 3.34 & 19.17 \\
\hline
\end{tabular}

*The bremsstrahlung losses plus the kE of plasma leaving through the divertor total. $3.5 \mathrm{MeV}$, i.e.. equal to the a particle energy/ fusion. 


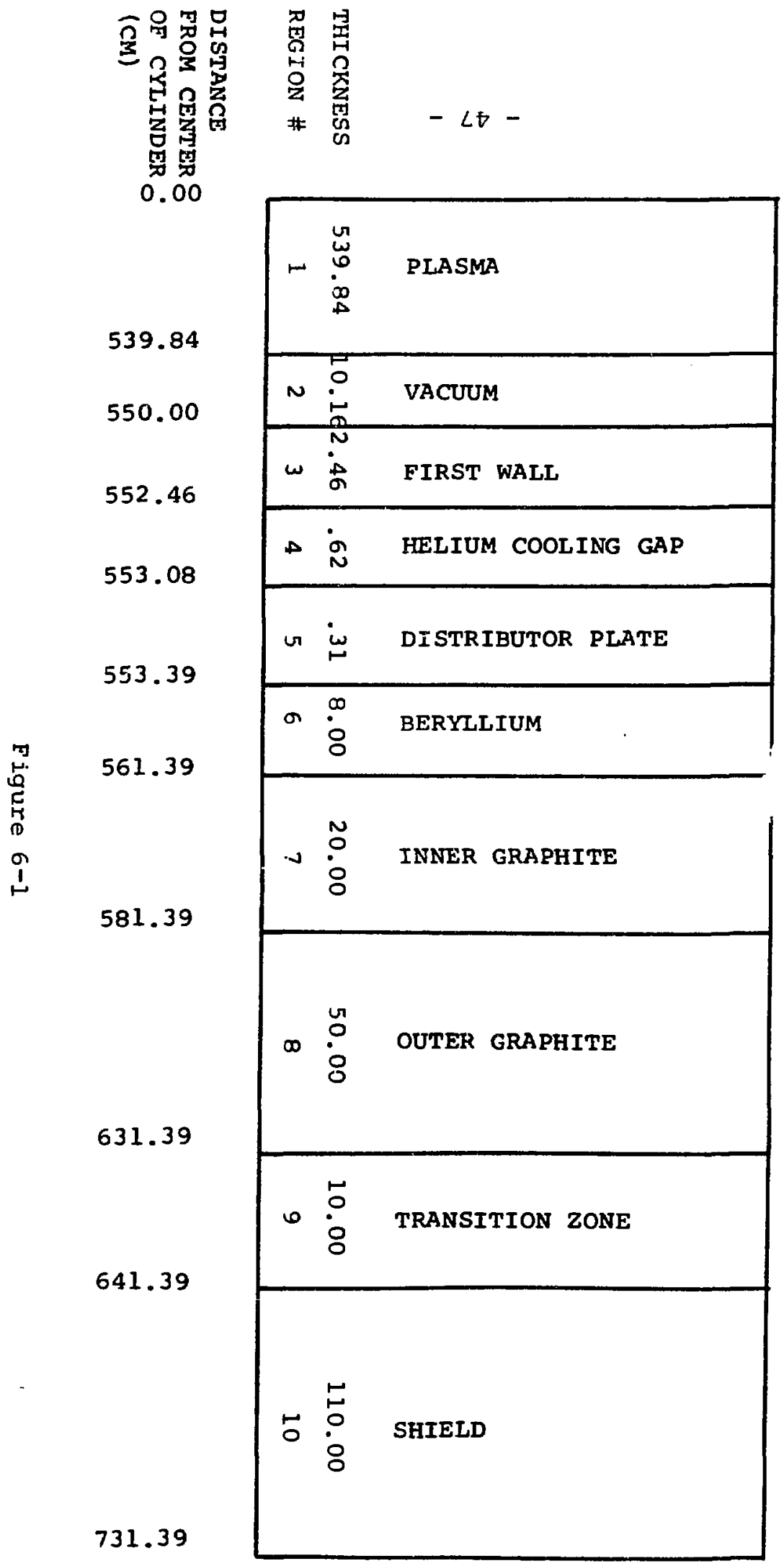




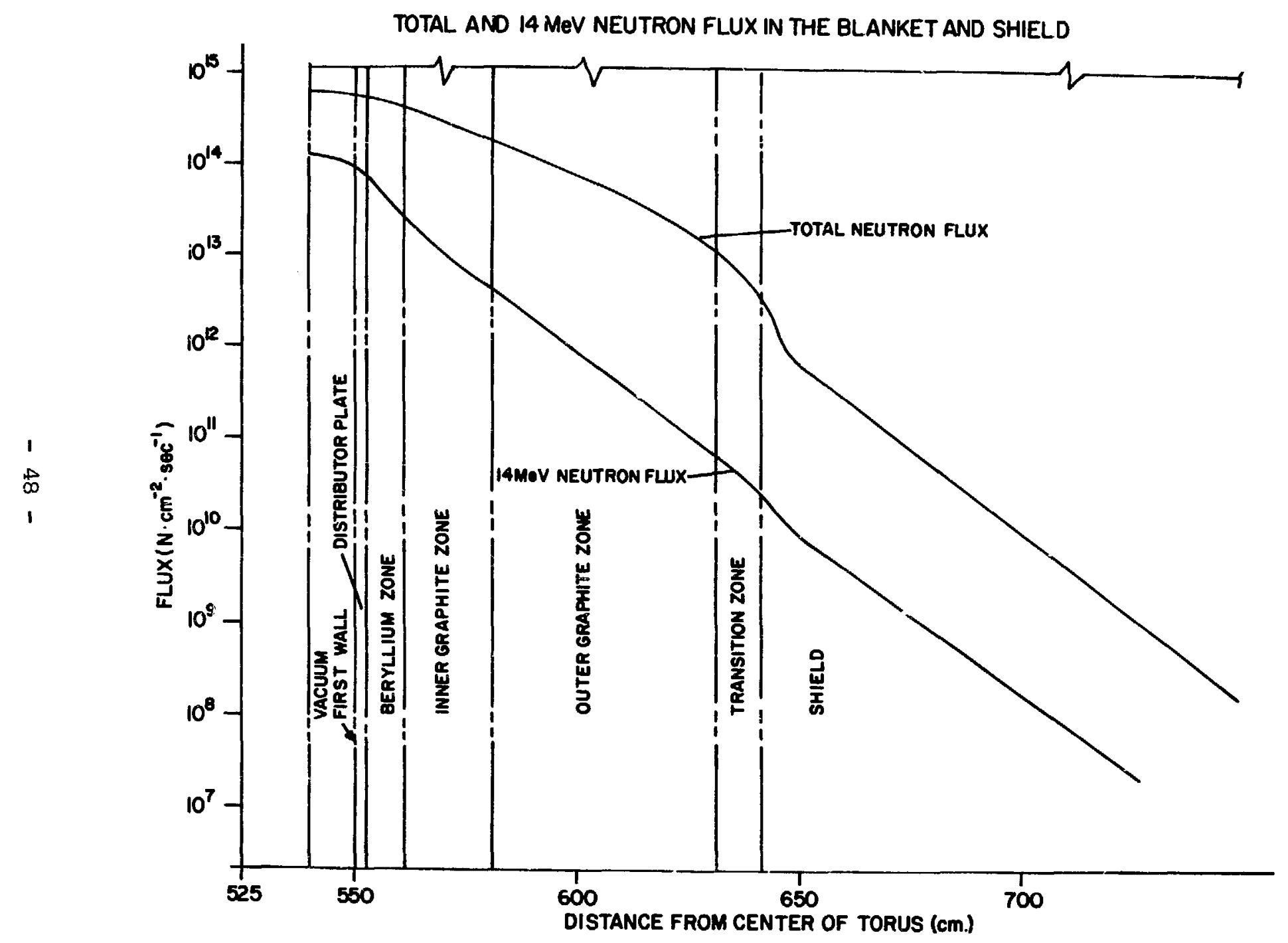

Figure 6-2 
ACTIVATION OF BLANKET AND SHIELD AFTER REACTOR SHUTDOWN

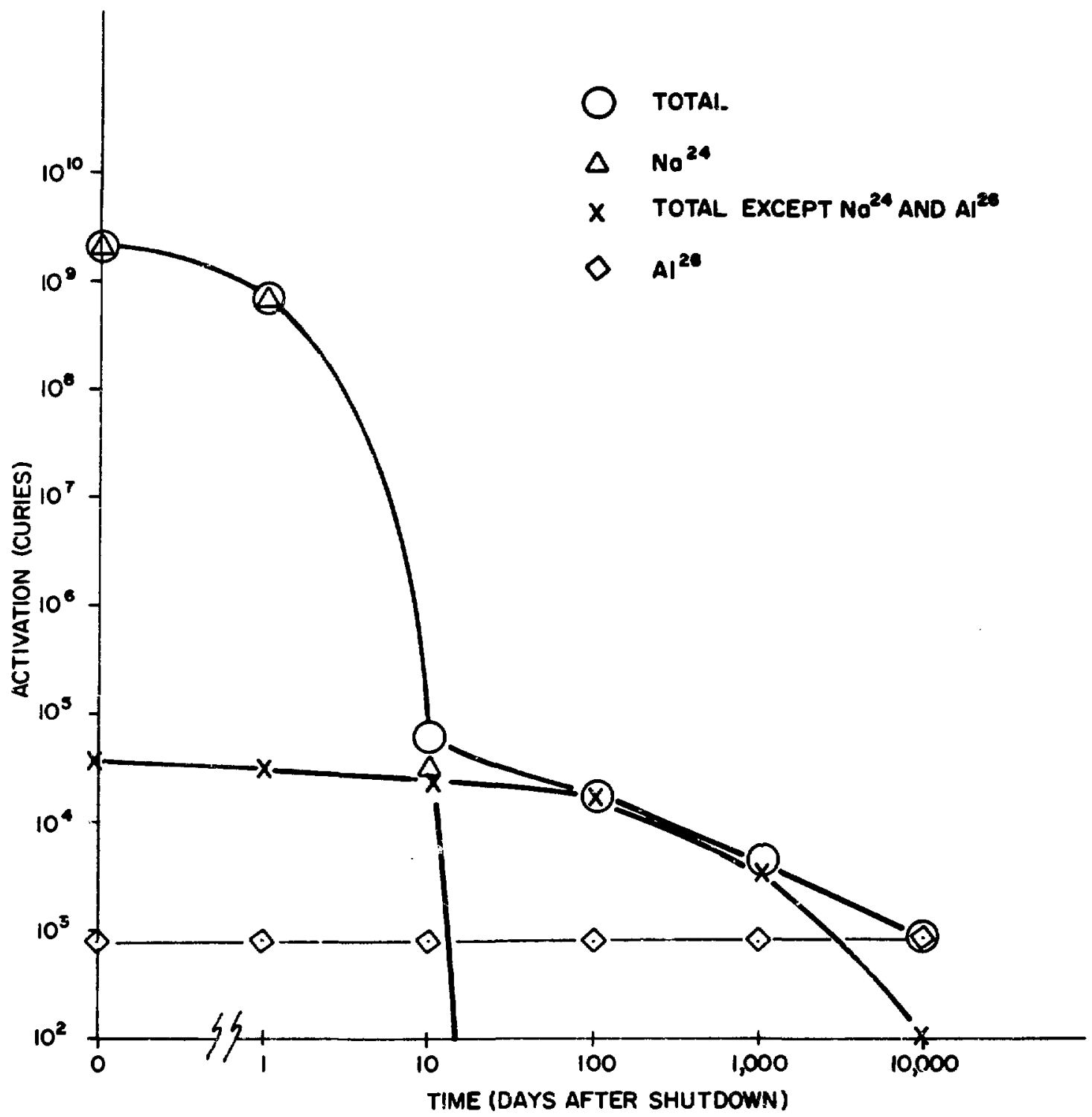

Figure 6-3 


\section{Blanket Cooling}

Two coolants, i.e., water and helium, are employed in the module design. In essence, the water coolant ducts are used to cool the Al module shell while the helium cools the hot neutron multiplying zone of Be balls and LiAl pellets as well as the stacked graphite rod region. In addition, the bred tritium diffuses into the helium coolant strearn. The water and helium coolant circuits may be found in Figure 5-3.

For the water coolant circuit the outlet temperature of the water is $400^{\circ} \mathrm{F}$ with water pressure of $750 \mathrm{psi}$. This is a considerable overpressure, designed to prevent boiling in the passages. It is probably much higher than needed for the very low power density. Cycle efficiency couid be substantially increased by reducing this overpressure. The $\Delta T$ across the module is $30^{\circ} \mathrm{F}$. All water coolant passages in the module shell are in parallel. Flow is down one-half of the module shell, across the dished head, and up the other half of the module shell. All told there are 84 inlet and 84 outlet passages. Figure 7-1 depicts the water pressure drop in the various regions as well as the mixed-mean or bulk water temperatures. For the given design condition, the flow is found to be turbulent, i.e., the Reynolds number is 31,000 . In addition, the volume rate of flow per coolant passage $i=0.485$ gallons/min. The convective heat transfer coefficient: is $1,411 \mathrm{Btu} / \mathrm{hr}$. $\mathrm{ft}^{20} \mathrm{~F}$ so that the difference between the "solid" surface temperature and water is small--approximately $26^{\circ} \mathrm{F}$ in the cold structure region of the Al module shell.

For the helium coolant circuit the inlet temperature is $350^{\circ} \mathrm{F}$ while the outlet is $1400^{\circ} \mathrm{F}$. The inlet pressure is 20 atm (absolute). Flow is down an annular channel on the inside of the module shell, through another annular gap between the dished head in the module shell and a dished flow distribution head, and then up through the 
inside of the module. Figure 7-2 depicts the helium pressure drops as well as bulk helium temperatures. In calculating these temperatures the heat leak from the hot to the cold region has been accounted for.

The helium flow down the annular channel on the inside of the module shell is found to be in the transition zone between laminar and turbulent flow, i.e., the Reynolds number is 6000 . The flow up the inside of the module is laminar $\left(\mathbb{N}_{\operatorname{Re}} \sim 1000\right)$ since the velocity decreases as a consequence of increased flow area relative to the flow down the annular channel and the kinematic viscosity increases with increasing helium temperatures. While this manifests itself in lower convective heat transfer coefficients compared with a fully turbulent case, the coefficients could be increased by inducing turbulence by some irregularities or projections along the graphite rods. This may not be necessary, since no thermal problems, in the sense of undue high temperatures in the graphite, seem to be present.

In the case when every sixth graphite rod or tube contains

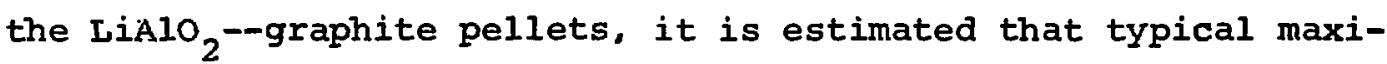
mum "fuel" temperatures in the inner and outer breeding zones are $1860^{\circ} \mathrm{F}$ and $2012^{\circ} \mathrm{F}$, respectively. (Actually slightly lower or higher values will be obtained depending on the radial position in the zones.) It might be noted that the maximum "fuel" temperatures decrease when the pellets are placed in more of the graphite rods, for example, every fourth or fifth rod, since the volumetric heat generation in the rod is then reduced.

The thermal balance for a module including the shield region is shown in Table 7-1. For both the helium and water circuits, pressure drops in the module and external circuits are shown in Table 7-l. 
Thermal Balance for Module and Shield

\section{Parameter}

Module Diameter, $\mathrm{cm}$

Total Module Power, KW(th)

Deposited Power in cold structure, $\mathrm{kw}(\mathrm{th})$

Deposited Power in Hot Structure, KW(th)

Power Removed from Cold structure, $\mathrm{KW}(\mathrm{th})$ 204.6

Power Removed from Bot strucuture, KW(th)

Power Deposited in Transition and Shield zone/ Module, KW (th)

Water Flow Rate, Ibs/sec per module

Helium Flow Rate, $1 \mathrm{bs} / \mathrm{sec}$ per module

Inlet water Temperature, ${ }_{F}$

outlet Water Temperature, ${ }^{\circ}$

Inlet He Temperature, ${ }_{F}$

Outlet Be Temperature, ${ }^{\circ}$

Pressure Drops/Module and External Circuits Module

Water, pressure drop through module*, psi 0.434

Beliur, pressure drop through module*, psi 0.275

Externai circuit

Total, psi

1. Cold structure refers to the inner and outer breeding zones: Al module shell, water, Al distributor plate, $\mathrm{Al}_{3} \mathrm{O}_{3}$ insulator: hot structure refers.to the neutrơn multiplying zone, etc.

2. Includes heat leak from hot to cold region. For detailed pressure drop distribution see Figs. 7-1 and 7-2. 




TYPICAL BLANKET MODULE

TEMPERATURE \& PRESSURE DROP PROFILE

$\mathrm{H}_{2} \mathrm{O}$ CIRCUIT

Figure 7-1 


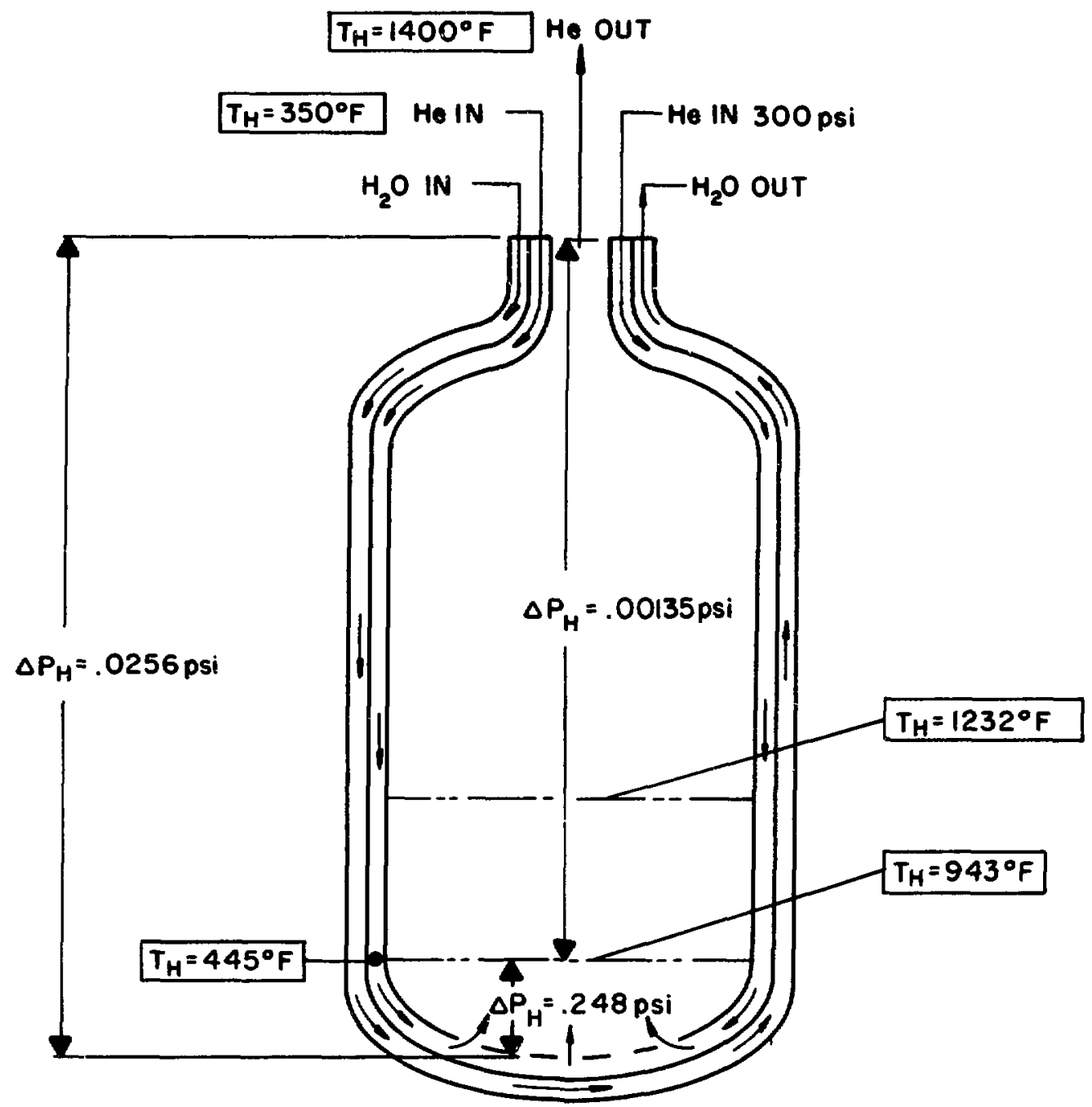

TYPICAL BLANKET MODULE

TEMPERATURE \& PRESSURE DROP PROFILE He CIRCUIT

Figure 7-2 


\section{Electricity Generation}

The CTR described in this report has two separate cooling systems, one for the first wall and another for the remainder of the blanket. The first wall cooling system involves pressurized water limited in temperatures to about $400^{\circ} \mathrm{F}$ and is nominally designed to absorb approximately $1 / 3$ of the total CTR heat output. The blanket cooling system uses helium gas at high temperature and is nominally designed to absorb the remaining $2 / 3$ of the CTR heat output.

Figure 8-1 is a flow sheet of the power cycle designed to convert the heat picked up in the water and in the helium to electricity for the nominal design. This flow sheet represents a combination of the process conditions reported for the summit Power Station of the Delmarva Power and Light Co. (1) (which involves a helium cooled fission reactor) and the comanche Peak Station of the Texas Utilities Generating co. (2) (which involves a PWR). Figure 8-1 shows approximately 9 million poinds/hr of helium being used to recover heat from the CTR blanket. The bulk of this helium which leaves the blanket at $1400^{\circ} \mathrm{F}$ is used to raise steam at $950^{\circ} \mathrm{F}$ and 2400 psia and to reneat the steam to $1000^{\circ} \mathrm{F}$. The steam conditions are identical to those of the Delmarva Plant. They are essentially the same as the steam conditions employed in many fossil fueled power plants. From the helium-steam boiler on to the steam condenser, only state-of-the-art technology is employed. The high temperature steam cycle operates at a net thermal efficiency of $37.4 \%$ with a condenser back pressure of 2.5" Hg. This is a relatively high turbine back pressure. A turbine back pressure of 1 " Hg or $1 \frac{1}{2} "$ ig would result in an improved cycle efficiency but would probably make dry cooling towers with reduced thermal pollution too expensive. 
First wall cooling as shown on Figure 8-1 is accomplished by approximately 169 million pounds per hour of water which enters at $370^{\circ} \mathrm{F}$ and leaves at $400^{\circ} \mathrm{F}$ and 750 psi pressure. This relatively low temperature water is flashed at a temperature of $370^{\circ} \mathrm{F}$ to produce approximately $6 \mathrm{million} 1 \mathrm{bs} / \mathrm{hr}$ of saturated steam at 173 psi pressure. The liquid leaving the flash drum is recycled to the first wall. The saturated steam leaving the flash drum is superheated to $500^{\circ} \mathrm{F}$ using approximately 380 thousand 1bs/hr of hot helium which is only about $5 \%$ of the hot helium from the blanket cooling circuit. This superheated steam drives a low pressure turbine to produce a net generation of $400 \mathrm{MW}$ of electricity at a net thermal efficiency of $22.2 \%$ for this portion of the cycle. From the exit of the superheater to the steam condenser, the process conditions are roughly equivalent to those of the comanche Peak plant. Here too, only stateof-the-art technology is employed.

The overall thermal efficiency of the combined cycle is $32 \%$ for the nominal design ( $1 / 3$ of fusion energy to low temperature region).

Table 8-1 shows a summary of the salient features of this combined high temperature-low temperature power cycle, for the nominal design. 
From the point of view of one interested in extracting useful power from a CTR, there are several important design parameters-some of which are dictated by considerations such as physics, metallurgy, etc., while others of which may be varied within limits so as to optimize selected characteristics, such as cycle efficiency.

The fraction of the total fusion energy that is picked up by the water circuit is the most important parameter affecting cycl.. efficiency. For a given design, neutronics dictates how such heat is deposited in the water circuit and how much is deposited in the remalnder of the blanket. Thus the cycle efficiency will vary with design. Figure 8-2 shows how the overall cycle efficiency depends on the heat split between the water circuit and the remainder of the blanket, based on all other cycle parameters being held constant. It can be seen from this figure that a $5 \%$ reduction in the heat deposited in the water circuit represents about a $1 \%$ improvement in the overall cycle efficiency. This proportionality holds over the entire range of interest for this concept.

There are several blanket design modifications that can raise overall net cycle efficiency from the $32 \%$ for the nominal design. (It should be noted, however, that the best cycle efficiency that could possibly be expected is the $\sim 38 \%$ characteristic of HTGR's with steam tubines. Benefits in efficiency resulting from design changes must, of course, be weighed agalnst increases in cost and/or decreases in reliability, if such occur.) such blanket design modification include:

1. Thinner aluminum module shells.

2. Cooling of $\mathrm{Al}_{2} \mathrm{O}_{3}$ insulating layers inside the modules 
with a portion of the helium coolant stream.

3. Placement of graphite curtain between the modules and the plasma, and re-radiation of the deposited bremsstrahlung energy to a non-water-cooled sink.

Thinner module shells can sinstantially increase overall cycle efficiency by reducing the amount of energy deposited in the shell. However, then either operating stress in the aluminum must become larger, or helium coolant pressure must drop and/or module diameter must decrease, if operating stress remains fixed. Some improvement in this direction appears possible, but better knowledge of material limits is necessary, together with more investigation of various alternate designs. In the present design, all of the helium flow is channeled down the annular space between the aluminum module shell and the inner reyion which contains the $\mathrm{Al}_{2} \mathrm{O}_{3}$ insulators and graphite. All energy deposited in the $\mathrm{Al}_{2} \mathrm{O}_{3}$ insulator by neutrons and gamme rays, as well as all thermal leakage through the insulator, ends up in the water circuit. It would be relatively simple to have part of the helium flow radialiy through the $\mathrm{Al}_{2} \mathrm{O}_{3}$ insulators into the hot graphite zone. In the fresent design, this would decrease the fraction of fusion energy removed by the water circuit from $32 \%$ to $25 \%$, increasing the overall cycle efficiency to $\sim 33.5 \%$.

The third design modification involves placing a graphite curtain (typically 1-2 in. thick) between the plasma and the aluminum module structure. Such a curtain, essentially a woven cloth of small diameter graphite fibers, has been proposed by the University of Wisconsin for their UWMAK-2 design (3). Besides protecting the first wall from plasma ion bombardment, the curtain substantially decreases the effect of impurities 
from the first wall on plasma performance. In the UWMAK-2 design, bremsstrahlung energy deposited in the surface of the curtain is radially conducted through the curtain and thermally radiated to the first wall. The curtain can be constructed to have a very low thermal conductivity in this radial direction, however, by using small diameter graphite yarns (e.g.. $\left.10^{-2} \mathrm{~cm}\right)$, loose weaves, and having the fibers predominantly lie in a plane normal to the radial direction. In this application, we would minimize radial conductivity, (a much easier task) while in the UWMAK-2 design, it would be maximized. A small portion (e.g., 15\%) of the blanket on the outer surface of the torus would then serve as a radiation sink, to receive thermally radiated energy from the inner surface of the graphite curtain. Over $90 \%$ of the bremsstrahlung energy could then be transferred to this radiation sink (a high tempeature tube bank, for example, using small diameter SAP tubes). This modification could reduce the fractional input to the water circuit to approximately $20 \%$.

With such design modifications in combination, it is expected that the overall fxaction of the fusion energy deposited in the water circuit can ultimately be reached to somewhere in the range of $10-15 \%$.

Further improvements in overall cycle efficiency can also be realized by improvements in process conditions. These include:

1. Higher exit temperature in water circuit.

2. Reduction of pressure in water circuit. (Total pressure is now assumed as 750 psia, giving an overpressure of almost 600 psia.

3. Higher superheater temperature in low pressure part of combined cycle. 
The exit water temperature for the water leaving the first wall represents a parameter whose upper limit is determined by materials considerations. In the design chosen for this study we have assumed that the use of aluminum alloys of the 6061 type (sans tree objectional alloying elements $\mathrm{Cr}$, Fe, etc.) would limit the maximum water temperature in the first wall to about $400^{\circ} \mathrm{F}$. Use of improved alloys or SAP should permit us to go to $475^{\circ} \mathrm{F}$ (perhaps even higher). In addition we have elected to operate with a water pressure of 750 psia to inhibit incipient boiling any place in the first wall. (This pressure may be much higher than necessary. Reduction would increase overall efficiency. This point is discussed more fully later.) Increasing the water discharge temperature from $400^{\circ} \mathrm{F}$ to $475^{\circ} \mathrm{F}$ results in more than tripling the heat pick up per pound of water circulated which permits a corresponding reduction in the amount of water circulated and the pumping power to achieve the circulation. As shown in Table $8-1$, roughly $20 \%$ of the gross electrical output of the low temperature cycle is consumed in the two condensate feed pumps. Reducing the water circulation rate by a factor of 3 has a very beneficial influence on the overall cycle efficiency. In one such case studied in detail, the overall cycle efficiency was as high as $33.6 \%$. Two other parameters which have a significant influence on the efficiency of the low temperature cycle are the amount of pressurization required to suppress incipient boiling and the amount of superheat employed. For the design shown in Figure 8-1, some 500 psi overpressure have been assumed which requires some 112 MW of electricity at the boiler feed pump. If the overpressure was 
reduced to 50 psi, i.e., a discharge pressure of 300 psia of 25 MW electricity would be required at the boiler feed pump. The 87 MW of electricity saved represents an increase in efficiency of the low pressure cycle to $27 \%$ and of the overall cycle to 34\%. We believe that with the relatively low heat fluxes involved in the water circuit, that the use of more than 50 psi overpressure is being too conservative. Subsequent designs will use much less overpressure.

Increasing the superheat temperature will also improve the cycle efficiercy. In the reference design shown in Figure 8-1, the flash steam is shown being superheated to only $500^{\circ} \mathrm{F}$. The helium used to superheat this steam was available at $1340^{\circ} \mathrm{F}$, hence the hot enci temperature difference in the superheater is $760^{\circ} \mathrm{F}$. This could be reduced 100 or more degrees without greatly effecting the size of the superheater while at the same time exerting a very beneficial effect on turbine efficiency (assumed at only 85\% isentropic) in the present design. Quantitative evaluation of this effect muste await more detailed calculations based on more reliable tyrbine expansion lines than those used in our preliminary studies. The graphs to follow are based on $475^{\circ} \mathrm{F}$ water. The trends they show with changes in process parameters are also valid for $400^{\circ} \mathrm{F}$ exit water, as evidenced by a number of spot-check calculations.

The remaining process parameters can for the most part bo set arbitrarily at values selected to optimize cycle efficiency. One of the most significant parameters was found to be the pressure selected for the flash drum. Lowering the pressure increased the pounds of steam obtained per pound of water circulated; however, the steam obtained was worth less in the power cycle. Increasing the flash pressure gave better steam conditions but resulted in less steam and a 
higher water circulation rate. Figure 8-3 shows the effect of flash drum temperature (pressure) on various cycle parameters contributing to cycle efficiency. The upper graph of Figure 8-3 shows an initial small increase in the overall cycle efficiency as the flash drum temperature is increased after which the cycle efficiency falls quite rapidly, with the low temperature cycle efficiency showing the most marked change.

The second graph of Figure 8-3 shows quite clearly how the total water circulation rate climbs, slowly at first and then very rapidly as the flash drum temperature approaches the temperature of the water leaving the first wall. The total vapor flow to the steam turbine varies only slightly. The lowest graph which shows the difference between the gross and net $\mathrm{kW}$ output as the shaded region labeled pump power clearly reflects this rapid rise in flow rate as the $\Delta T$ in the flash drum vanishes and furthermore indicates why the cycle efficiency falls as it does.

The third graph on Figure 8-3 shows the superheater duty falling at. first as the flash drum temperature is increased and then finally rising quite steeply. This effect is quite significant since the superheat duty represents thermal energy taken from the high temperature portion of the cycle where its conversion to kilowatts is effected at higher efficiency. We would therefore expect optimum overall plant performance to occur near the minimum in this curve.

Figure 8-4 is similar to the upper graph of Figure 8-3, and shows in addition the efrect of the first wall exit temperature. The need to operate the first wall cooling system at as high a temperature as is practical is clearly evident 
from this figure. Again it should be noted that this effect assumes reduced importance as the fraction of the total heat deposited in the first wall is reduced.

In summary, it appears that there are a number of promising routes that can substantially increase overall cycle net efficiency from the $32 \%$ calculated for the design shown in this study. It appears quite possible to achieve an overall net efficiency of $35 \%$, and with detailed design and optimization, perhaps $36 \%$. 
Table 8-1

\section{Summary of Power Cycle process \\ Conditions}

Nominal Design ( $1 / 3$ Input to Low Temperature)

CTR Thermal Output

First Wall

Blanket region

Total Thermal Output

$\begin{array}{r}5.63 \times 10^{9} \mathrm{BTU} / \mathrm{Hr} \\ 11.49 \times 10^{9} \mathrm{BTU} / \mathrm{Hr} \\ \hline 17.12 \times 10^{9} \mathrm{BTU} / \mathrm{Hr}\end{array}$

High

Temperature Circuit Temperature Circuit

Steam flow, lbs/Hr

Steam temperature, ${ }^{\circ}$

$8.5 \times 10^{6}$

$6.2 \times 10^{6}$

$950 / 1000$

500

$2515 / 586$

150

Steam Pressure, psia

Gross Turbine Output, BTU/Hr

HP Turbine $8.1 \times 10^{8}$

Helium Cixculation Turbine $2.4 \times 10^{8}$

LP Turbine $1.2 \times 10^{9}$

Boiler Feed Pump Turbine $1.0 \times 10^{8}$

LP Turbine $1.8 \times 10^{9} \quad 1.8 \times 10^{9}$

Gross Electrical Generation, MW 1208.5

Boiler Feed Pumps, MW

0.5

Net Electrical Generation, MW

1208

510.6

Total

MW

Total Fusion Thermal Power

Overall efficiency

$113.6 *$

397

1605 (both circuits)

$5000 \mathrm{MW}(\mathrm{th})$

$32 \%$

*This power loss is due to high overpressure in the water coolant circuit. It should be possible to reduce this term to $\sim 30 \mathrm{MW}$. 


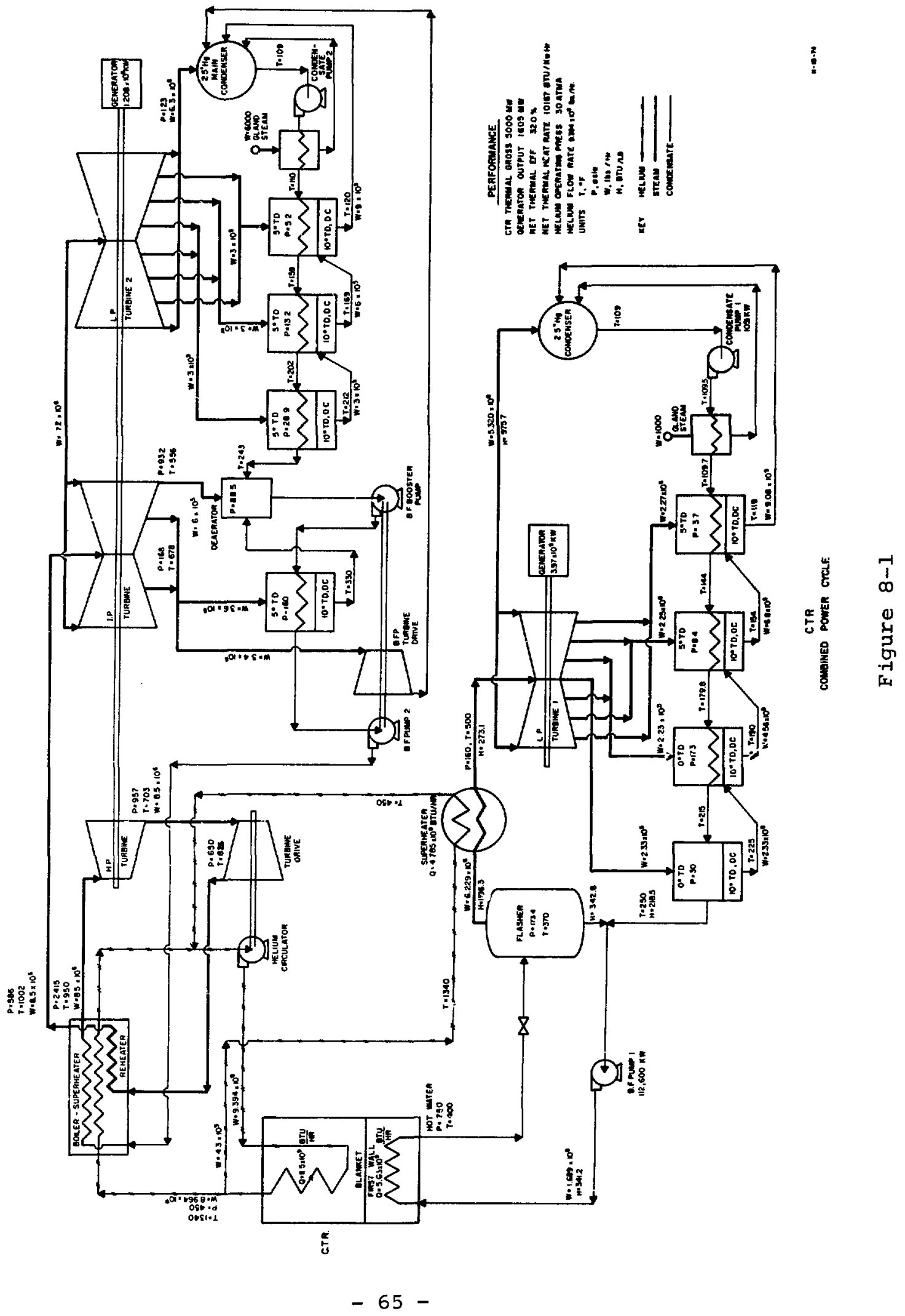


EFFECT OF CHANGING PERCENT OF TOTAL

CTR HEAT OUTPUT WHICH GOES TO WATER

CIRCUIT ON OVERALL EFFICIENCY OF THE COMBINED CYCLE

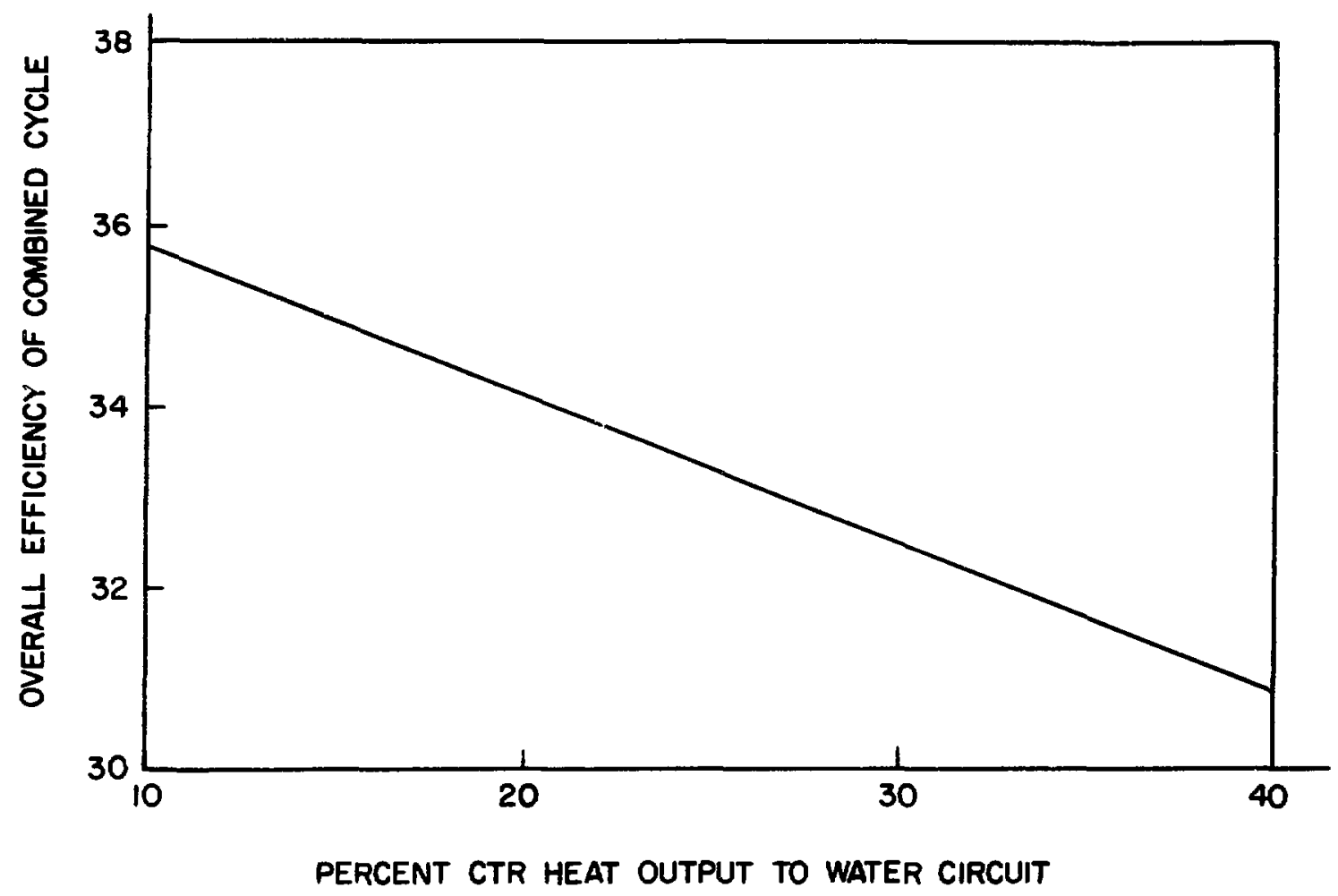

Figure $8-2$ 
EFFECT OF FLASH DRUM

TEMP ON VARIOUS CYCLE

PARAMETERS CONTRIBUTING TO CYCLE EFFICIENCY

FIRST WALL EXIT $T=475^{\circ} \mathrm{F}$ FEED WATER PREHEAT TEMP $=350^{\circ} \mathrm{F}$

FRACTION CTR OUTPUT TO FIRST WALL $=0.333$ SUPERHEAT $T E M P=660^{\circ} \mathrm{F}$ CONDENSER TEMP $=109^{\circ} \mathrm{F}$
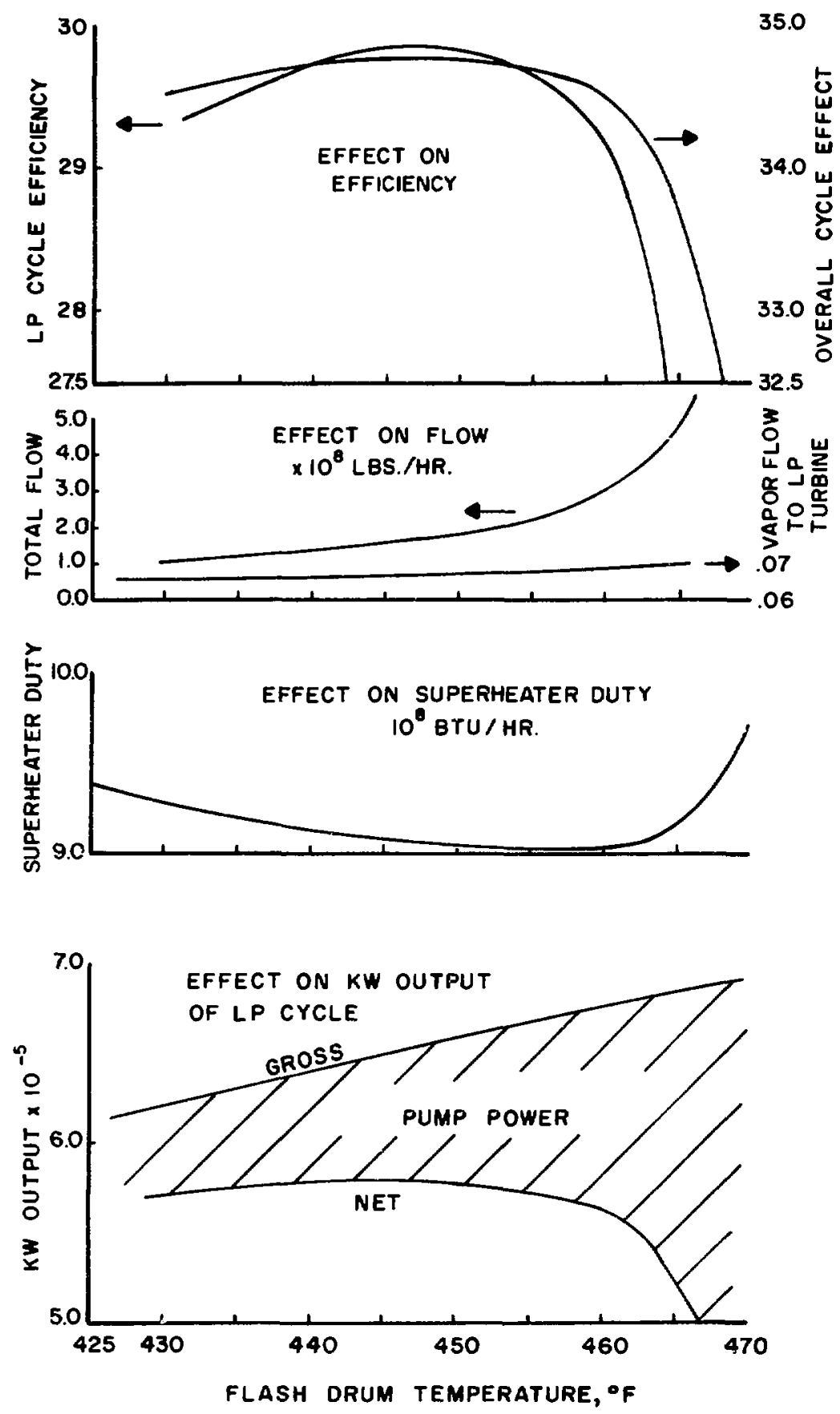

Figure $8-3$ 


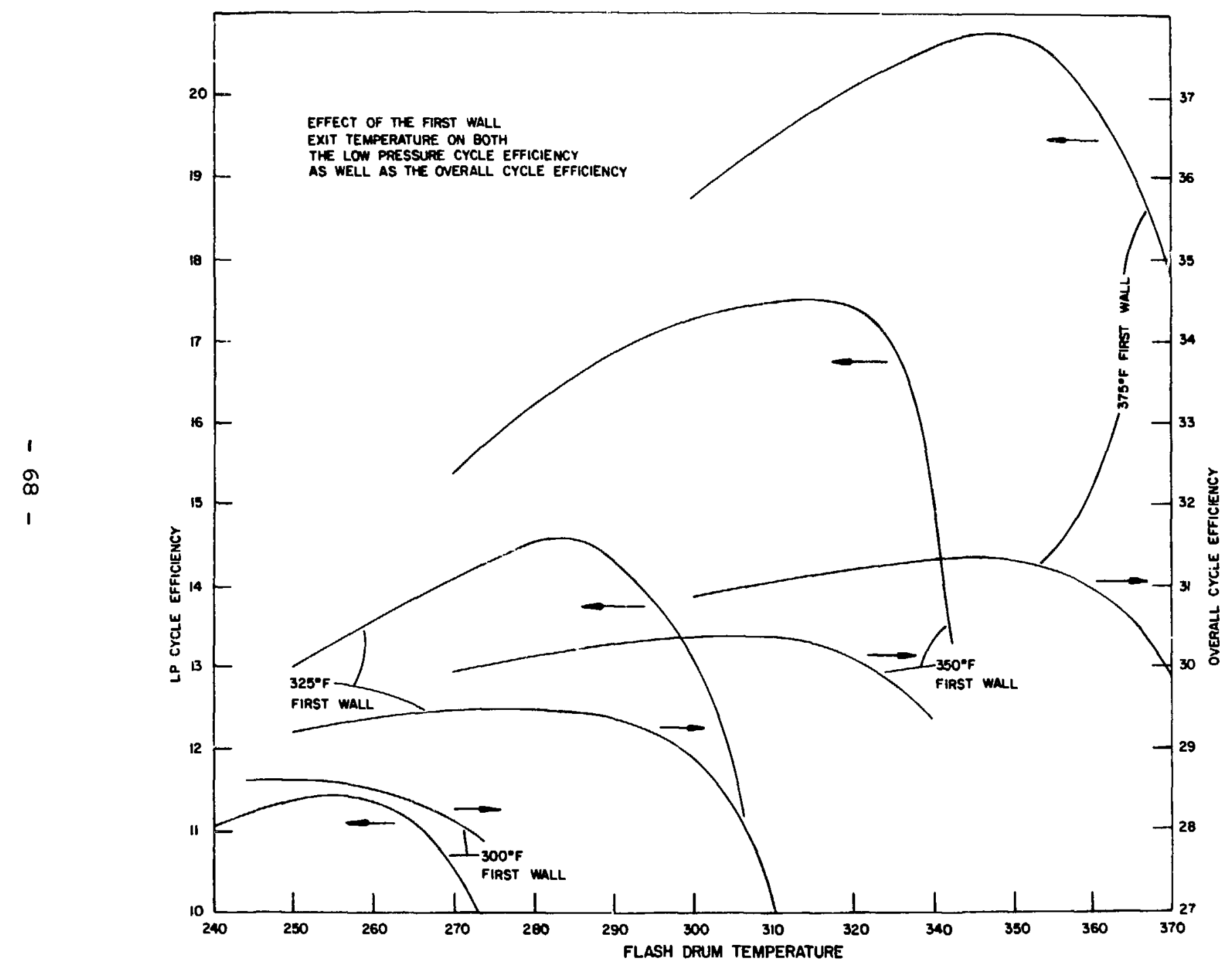

Figure 8-4 


\section{Recovery of Spent Fuel and Plasma Exhaust}

The partially burned fuel, consisting of deutcrons, tritons, $\mathrm{He}^{4}$ particles, and impurity ions is exhausted through the upper and lower divertor slots. In the UWMAK-1 design (I) the hot ions (T 10 KeV) strike a series of liquid lithium films and are trapped (except for He) and recovered by circulation and processing of the liquid lithium. The presence of liquid lithium in a reactor with water cooled aluminum modules is not desirable.

The divertor surfaces in this design are assumed to be similar to the first walls considered for the FERF design (2). The plasma ions strike solid tubes (e.g. SAP) giving up their energy to a coolant inside the tubes. The surface of the tube is coated with a substance with high diffusivity for hydrogen, e.g., palladium, so that they may rapidly diffuse out. The required vacuum is maintained by having high capacity cryopumps behind the divertor surfaces to trap the neutral gases as they leave the tube surface. The cryopumps are essentially black, i.e., absorb all incident atoms and molecules, so that the pumping speed of the divertor region is very high. The thermal load on the cryopump due to absorption of the spent fuel is very small (i.e., about $10^{-4} \%$ of the reactor thermal output), since virtually all of the ion energy is deposited as heat on the divertor surfaces. Divertor surfaces may have to be periodically replaced in a manner similar to the blanket modules. 


\section{Recovery of Bred Tritium}

There are three modes of tritium recovery from solid lithium compounds :

(a) Letting the brea tritium diffuse into the helium coolant stream, from which it is trapped out.

(b) Letting the bred tritium diffuse into the vacuum region between the plasma and the first wall, and recovering it along with the plasma exhaust.

(c) Letting the bred tritium diffuse into a separate tritium recovery stream which is not part of the coolant circuit.

Mode (a) is chosen for this design. Its chief advantages are: simplicity of blanket design, as contrasted with mode (c); and higher thermal conversion efficiency, since the $4.8 \mathrm{MeV}$ per neutron absorption in $\mathrm{Li}^{6}$ can be deposited into the high temperature helium coolant, as contrasted to mode (b) where the $4.8 \mathrm{MeV}$ must be deposited in the cool aluminum structure. The chief disadvantages are the burden of tritium in the circulating helium coolant, and the tritium diffusion into steam circuits through steam generator tubes. However, these problems can be overcome by suitable design, while the benefits of increased thermal efficiency and greater blanket simplicity with mode (a) are important. Figure 10-1 illustrates the overall flow sheet for tritium release to and recovery from the helium coolant circuit. At steady state, the release rate from the blanket equals the breeding rate and also equals the recovery rate in the tritium trap. The tritium concentration in the helium coolant is given by

$$
\mathrm{Y}\left(\mathrm{T}_{2}\right)=\frac{\dot{\mathrm{N}}_{\mathrm{T}_{2}}}{\mathrm{~N}_{\mathrm{He}}} \frac{\tau_{\mathrm{He}}}{\mathrm{F}_{\mathrm{p}}(1-\mathrm{R})}
$$

where $1-R$, the fractional recovery of the tritium trap, is given by 


$$
1-R=1-\frac{Y\left(T_{2}\right) \text { exit }}{Y\left(T_{2}\right) \text { inlet }}
$$

with $\mathrm{R}$ expected to be much $\ll 1$ (very efficient trap). We define:

$$
\begin{aligned}
\mathrm{y}_{\left(\mathrm{T}_{2}\right)}= & \text { atom fraction of tritium in the helium coolant } \\
\mathrm{N}_{\mathrm{He}}= & \# \text { of } \mathrm{g} \text { moles in the helium coolant circuit } \\
\tau_{\mathrm{He}}= & \text { mean circulation time for helium in coolant circuit, } \\
& \text { seconds } \\
\mathrm{f}_{\mathrm{p}}= & \text { fraction of helium coolant stream sent to tritium } \\
\dot{\mathrm{N}}_{\mathrm{T}_{2}}= & \text { trap }
\end{aligned}
$$

Tritium lost to the reactor containment building can be neglected in this balance, since the loss corresponding to one curie/ day leakage is $\sim 10^{-5} \%$ of the bred tritium.

For a fixed blanket coolant circuit design, tritium breeding ratio, and reactor power level, the tritium inventory in the helium coolant circuit is only a function of $f_{\mathrm{p}}$, the processing fraction. For this study, the helium inventory is $\sim 20,000 \mathrm{lbs}$, the mean circulation time is $\sim 5$ seconds, the tritium breeding ratio is 1.00 (assumed to be spoiled to this value from the estimated value of 1.20 ), the breeding rate of tritium in the blanket is $1.3 \times 10^{-3}$ $g$ moles/sec, and $R=0$. The tritium inventory in the helium circuit is then given by

$$
C\left(T_{2}\right)=\frac{360}{f_{p}} \text { curies. }
$$

For the design value for processing fraction of 0.05 (5\% of the coolant stream is sent to the tritium trap), the total blanket inventory is only 7200 curies. This could be reduced to 3600 curies with a processing fraction of $10 \%$, which should be achievable with not too much difficulty.

The helium coolant system is divided into 12 separate circuits, one for each magnet sector. The tritium inventory in the helium in 
each circuit is then only 600 curies.

The tritium inventory in the blanket is given by

$$
\mathbf{N}_{\left(T_{2}\right) \text { Blanket }}=\dot{N}_{\left(T_{2}\right)} 6 \times 10^{4} \tau_{B}
$$

where $\tau_{B}$ is the mean holdup time of tritum in the blanket, in seconds. $\tau_{B}$ will depend on many factors, such as the composition of the solid lithium compound and its temperature, which affects the equilibrium pressure of tritium above the solid as well as the diffusion rate of tritium through the solid; the solid lithium compound particles size and porosity, which affects the diffusion rate; the presence or absence of added protium $\left(\mathrm{H}_{2}\right)$ or deuterium $\left(D_{2}\right)$ in the helium coolant, which effects the equilibrium pressure of tritium above the solid (the presence of $\mathrm{H}_{2}$ or $\mathrm{D}_{2}$ increases the equilibrium pressure of tritium); and the amount of solid lithium compound in the blanket, which affects the tritium inventory if $\tau_{B}$ is controlled by equilibrium processes.

A substantial number of experiments have been carried out at BNL (1) on the extraction of tritium from solid lithium compounds as a function of composition, temperature, particle size, helium flow rate, and amount of added $\mathrm{H}_{2}$ 。 The tritium is produced in-situ in the solid lithium compounds by neutron irradiation of a sample to about the same concentration as would exist in the reactor and is then batch extracted by flowing helium under known conditions.

Extraction from LiAl is readily accomplished at temperatures of 400-500 $\mathrm{C}$ from 10-20 mesh particles with a mean holdup time of $\sim 0.5$ hour. The extraction seems to be controlled by diffusion of tritium in the LiAl particles, since smaller particle sizes result in faster extraction at the same temperature. The equilibrium pressure of tritium above solid lithium compounds is generally many orders of magnitude greater than that above liquid lithium at the same temperature. A lower limit for the sievert's constant for 
LiAl can be derived from the experiments; on the basis of this limit $\left(\mathrm{k}_{\mathrm{s}} \geq 1.2 \times 10^{4}\right.$ torr $^{1 / 2} /$ atom fraction at $\left.500^{\circ} \mathrm{C}\right)$ a mean tritium holdup time of 0.5 hour for the blanket design described in this study is well within the diffusion controlled domain for tritium extraction. We take the mean tritium holdup time in LiAl to be 0.5 hour, which then corresponds to a LiAl particle size on the order of 1 millimeter.

The LiAl balls in the neutron multiplication region are 1 centimeter in diameter. The balls will be formed by compacting LiAl particles of $\sim 1$ millimeter diameter with an inert binder, probably beryllium particles. A porous stxucture will be retained throughout the ball. Diffusion of tritium in the gas phase is rapid enough (characteristic diffusion times are a few seconds) that tritium will quickly escape to the main coolant stream once it leaves the LiAl particles. Fines from particle abrasion are retained in the module.

Fewer results are available with high temperature lithium ceramics $\left(\mathrm{IiAlO}_{2}, \mathrm{LiSO}_{3}\right)$, but the controlling process for tritium release again appears to be diffusion, not equilibrium. However, the particle temperature must be higher than that necessary for LiAl and/or the particle size must be smaller. A mean tritium holdup time of 0.5 hour is again assumed for the $\mathrm{LiAlO}_{2}$. The particle size is then determined by the temperature, which will vary from $\sim 500^{\circ} \mathrm{C}$ to $800^{\circ} \mathrm{C}$ in the graphite region. (Though we have specified $\mathrm{LiAlO}_{2}$ as the solid lithium breeding material in the graphite region, it seems likely that LiAl would actually be used in the cooler parts of the region, with $\mathrm{LiAlO}_{2}$ in the hotter parts $\left.\left(>600^{\circ} \mathrm{C}\right)\right)$. Particle diameters of $\sim 30$ microns appear satisfactory. The $\mathrm{LiAlO}_{2}$ particles would be mixed with an inert material, probably graphice powder, and compacted into porous pellets, which would then be inserted into hollow graphite tubes with holes in their walls. As with the LiAl balls, the gas phase diffusion 
rates are sufficiently fast that tritium holdup time in the holes and cracks is short compared to holdup time in the LiAlo 2 particles.

Iisio $_{3}$ appears to be a satisfactory breeding material and could be used in place of $\mathrm{LiAlO}_{2}$. It has a considerably shorter holdup time for equivalent particle sizes and temperature.

For a mean tritium holdup time of 0.5 hour, the total tritium blanket inventory is 140,000 curies. This can probably be reduced by a factor of 3 or 4 using smaller particles. However, an inventory of 140,000 curies seems acceptable, since the tritium is in solic material, and it would be released extremely slowly in a major accident. The diffusion coefficient of tritium in a solid lithium compound will be many orders of magnitude smaller at ambient temperature.

There are at least three practical methods of recovering tritium from the helium coolant stream:

(a) Absorption of tritium gas in a metal hydride,

(b) Conversion of tritium to $\mathrm{T}_{2} \mathrm{O}$ through controlled additions of oxygen, with subsequent absorption or cold trapping,

(c) Cryopumping $\mathrm{T}_{2}$ out of the helium stream.

Method (a) has been selected for this design since i.t does not raise questions about the effect of $\mathrm{T}_{2} \mathrm{O}$ or $\mathrm{O}_{2}$ on blanket materials, and should result in cheaper and smaller trapping units than method (c). (Method (c) can be made energy efficient by regenerative heat exchange, but substantial heat exchange surface is needed.)

Figure 10-2 illustrates how hydride beds would be used to recover tritium from the helium process stream, which has a flow rate of $5 \%$ of the total helium flow. The helium process streams go through the hydride beds at $500^{\circ} \mathrm{C}$ where $\mathrm{T}_{2}$ is absorbed and then are returned to the main coolant circuits by He circulators. (There are 12 helium circuits for the reactor, each with its own processing loop.) Absorption takes place for 5 minutes, and the bed is 
then desorbed for 5 minutes of $\mathrm{T}_{2}$ by cryopumping. The He process stream is isothermal--it enters and leaves at $500^{\circ} \mathrm{C}$.

Two beds are provided for each of the 12 circuits. There will be a number of backup bed circuits able to take over in case of failure. The number of backup circuits is not determined, but probably will be on the order of six.

The total volume of the absorbing bed (metal hydride particles plus voids) is given by

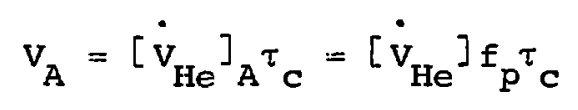

where

$$
\begin{aligned}
\mathrm{v}_{\mathrm{A}}= & \text { volume of absorbing bed } \\
\mathrm{f}_{\mathrm{p}}= & \text { process stream fraction of main flow }\left(\mathrm{f}_{\mathrm{p}}=0.05\right) \\
\tau_{\mathrm{c}}= & \text { superficial contact time in absorbing bed, seconds } \\
\dot{\mathrm{V}}_{\mathrm{He}}= & \text { flow rate of main helium coolant stream, } \mathrm{m}^{3} / \mathrm{sec} \\
& (1 / 12 \mathrm{th} \text { of reactor). }
\end{aligned}
$$

Specification of $\tau_{c}$ is sufficient to $f_{i x}$ the absorbing bed volume, while specification of one other variable (flow area, flow velocity, or contact length) is sufficient to $f$ ix the other bed variables through the relations

$$
\mathrm{v}=\left[\mathrm{v}_{\mathrm{He}}\right]_{\mathrm{A}} / \mathrm{A}_{\mathrm{f}}=\frac{\Delta \mathrm{z}}{\tau_{\mathrm{C}}}
$$

The actual contact time, ${ }^{{ }^{*}}{ }^{*}$, is less than the superficial contact time, by the relation

$$
\tau_{c}{ }^{*}=\tau_{c} \frac{\text { void volume }}{\text { total bed volume }}
$$

where the total bed volume includes the voids between particles, and $\mathrm{v}=$ svperficial contact velocity, $\mathrm{m} / \mathrm{sec}$

$A_{f}=$ superficial flow area, $m^{2}$

$\Delta \mathbf{z}=$ bed thickness, $m$

Generally $\tau_{c}{ }^{*}$ will be about $40 \%$ of $\tau_{c}$. 
The next step is to select a particular metal hydride for the absorbing-desorbing beds. Figure 10-3 shows how some of the possible choices meet the process requirements. For a $5 \%$ process flow, the partial pressure of $\mathrm{r}_{2}$ in the entering process stream is $10^{-3}$ torr, assuming that the exit stream partial pressure is zero.

It is evident that cerium and yttrium hydride are too stable to be suitable for a tritium absorbing bed, while zirconium and titanium appear to be good choices. The metal hydride not on?y has to readily absorb tritium, but aiso has to release it when cryopumped. Thus the ideal hydride has an equilibrium pressure of $\mathbf{T}_{2}$ above it which is not too far below the partial pressure of $\mathbf{T}_{2}$ in the helium process stream.

The vertical dashed lines in Figure 10-3 represents the average concentration of tritium present in the titanium or zirconium bed. This can be derived from the following relationships

$$
\begin{aligned}
& \Delta C=\frac{2\left(\dot{N}_{T_{2}^{\prime}}\right) \tau_{A} \times 100}{12\left(V_{A}\right) f_{m}(\rho / A)_{m} \times 10^{6}} \\
& C=\frac{\Delta C}{(\Delta C / C)} \text { design }
\end{aligned}
$$

where

$$
\begin{aligned}
& \Delta \mathbf{C}=\text { concentration change of tritium in absorbing metal }
\end{aligned}
$$



$$
\begin{aligned}
& \dot{\mathrm{N}}_{\mathrm{T}_{2}}=\begin{array}{l}
\text { total breeding rate of tritium in reactor (all } 12 \\
\text { circuits), } \mathrm{g} \mathrm{mole} / \mathrm{sec}
\end{array} \\
& \mathrm{v}_{\mathrm{A}}=\text { volume of absorbing bed, } \mathrm{m}^{3} \text { (including voids) } \\
& (\mathrm{P} / \mathrm{A})_{\mathrm{m}}=\text { density }(\mathrm{g} / \mathrm{cc}) / \text { atomic weight for metal hydride } \\
& \begin{aligned}
\Delta \mathrm{c} / \mathrm{C} \text { design }= & \text { fractional change of concentration of } \mathrm{T} \text { in } \\
& \text { metal hydride during absorption cycle. }
\end{aligned} \\
& \mathrm{f}_{\mathrm{m}}=\text { volume fraction of metal hydride particles. }
\end{aligned}
$$


For the dashed lines in Figure 10-3, the following representative values were used:

$$
\begin{aligned}
& { }_{C}=0.5 \text { seconds (this is discussed late } \tau \text { ) } \\
& \dot{\tau}_{A}=300 \text { seconds } \\
& \dot{\mathrm{N}}_{\mathrm{T}_{2}}=1.3 \times 10^{-3} \mathrm{~g} \text { moles } / \mathrm{sec} \\
& (\rho / A)_{\mathrm{m}}=0.1 \text { (titanium) } \\
& \mathrm{V}_{\mathrm{A}}=3.0 \mathrm{~m}^{3}\left(\mathrm{~T}_{\mathrm{C}}=0.5 \mathrm{sec}, \mathrm{p}=20 \mathrm{~atm}, \mathrm{~T}=500^{\circ} \mathrm{C}, \mathrm{f}=0.05,\right. \\
& \left.\quad \text { and } \dot{\mathrm{N}}_{\mathrm{He}}=4.5 \times 10^{5} \mathrm{~g} \text { moles } / \mathrm{sec}\right)
\end{aligned}
$$

Under these conditions and with the bed composed wholly of titanium particles ( $\left.f_{m}=0.4\right)$

$$
\Delta C=5.4 \times 10^{-5} \text { atom } \%
$$

choosing $\Delta C / \bar{C}=0.27$, then $\bar{C}=2 \times 10^{-4}$ atom \% which appears to be too low for easy $T$ recovery from the hydride bed. The right-hand dashed line shows $c=2 \times 10^{-2}$ atom percent, which is achieved by if $99 \%$ of the particles in the bed are inert ballast (e.g.. $\mathrm{Al}_{2} \mathrm{O}_{3}$ ) while the remaining $1 \%$ are metal hydride in an intimate mixture. The inert particles serve to thicken the bed and achieve the desired contact time, which appears to be controlled by gas phase diffusion rates for tritium in He, rather than by reaction rates at the solid metal hydride. This would appear to be the best design approach for a tritium absorbing bed with titanium.

A wide variety of desirable combinations is possible, however, with different $\triangle \mathrm{C} / \mathrm{C}$ and different amounts of inert material. The point that should be noted is that the concentration swing of $T$ in the metal hydride should be small ( $<0.3$, for example) and the resultant maximum equilibrium pressure of $\mathbf{T}$ above the metal hydride somewhat below the minimum partial pressure desired for $T_{2}$ in helium。

For purposes of this design, the hydride bed particles are 
assumed to be $99 \%$ inert material and $1 \%$ titanium, with an overall void fraction of $40 \%$ 。

Because of the small particle size (typically $2 \times 10^{-2} \mathrm{~cm}$ ), ana the low helium flow velocities (superficial velocity is $\sim 10 \mathrm{~cm} / \mathrm{sec}$ ). the flow in the bed is laminar $\left(\mathrm{N}_{\mathrm{Re}} \sim 10^{-1}\right)$. Assuming gas phase diffusion controls absorption rate, the characteristic diffusion time for tritium in the gas film is approximated by

$$
\tau_{D} \sim \frac{D_{D}^{2}}{4 D_{V}}
$$

where

$$
\begin{aligned}
& \mathrm{D}_{\mathrm{p}}=\text { particle diameter, } \mathrm{cm} \\
& \mathrm{D}_{\mathrm{V}}=\text { diffusion coefficient of } \mathrm{T}_{2} \text { in } \mathrm{He}, \mathrm{cm}^{2} / \mathrm{second}
\end{aligned}
$$

Taking $D_{p}=2 \times 10^{-2} \mathrm{~cm}$ and $D_{V} \cong 0.2 \mathrm{~cm}^{2} / \mathrm{second}(\mathrm{p}=20 \mathrm{~atm}, \mathrm{~T}=$ $\left.500^{\circ} \mathrm{C}\right), \tau_{0} \sim 0.5 \times 10^{-3}$ second, the actual contact time, $\tau_{c}^{*}$, is $0.4 \times 0.5$ or 0.2 seconds, which is 400 times $\tau_{D^{*}}$ It thus appears that the assumption of $\tau_{c}=0.5$ second is quite conservative, and that the absorbing beds in an actual reactor will probably be considerably smaller than shown in this design.

The absorption of $\mathrm{T}_{2}$ in titanium is exothermic and the desorption endothermic, with a heat of reaction of $\sim 20 \mathrm{Kcal} / \mathrm{g}$ atom of $\mathrm{H}_{2}$. Because of the very small concentration change auring absorption and desorption, however, the bed temperature change is very small, i.e., on the order of $0.01{ }^{{ }^{K}} \mathrm{~K}$. Tris will have no effect on the kinetic or equilibrium properties of the bed.

Gulbransen and Andrew (2) have measured the kirietics of hydriding of $\mathrm{H}_{2}$ for $15 \mathrm{mil}$ titanium sheets as a function of $\mathrm{H}_{2}$ pressure and hydriding temperature. As with the equilibrium pressures of $\mathrm{H}_{2}$ above metal hydrides, we assume that kinetics of $\mathrm{T}_{2}$ and $\mathrm{H}_{2}$ hydriding are essentially the same. Even at $300^{\circ} \mathrm{C}, 15 \mathrm{mil}$ 
titanium sheet could absorb tritium substantially more rapidly than would be required in the absorbing bed $\left(P\left(T_{2}\right)=10^{-3}\right.$ torr, $\tau_{A}=300 \mathrm{sec}, 99 \%$ inert solids). Extrapolated to $500^{\circ} \mathrm{C}$, the absorbing rate would be orders of magnitude faster. In addition, the titanium particles in the bed will be smaller spheres ( 8 mils dianeter), and this will further increase hydriding rate. It thus appears that gas phase diffusion of $T_{2}$ in helium will be controlling, and not the reaction of $\mathrm{T}_{2}$ with titanium.

Bea parameters are summarized in Table 10-1 and a drawing of a typical absorbing bed is shown in Figure 10-4. Note that the helium flows in parallel through a number of horizontal trays holding the absorbing titanium and the inert solids.

Figure 10-5 shows an overall tritium map for the reactor. I represents the inventory of tritium in curies, and $Q$ the tritium flux in curies/day. The inventories in the solid blanket, helium circuit, hydride beds, and cryopump, are relatively small and can be reduced by perhaps a factor of two or three. The methods of reduction would include: smaller particles of solid lithium compound in the blanket, to reduce blanket holdup time by two or three times; shorter absorption times and smaller hydride beds: and larger processing flow rate (10\% instead of $5 \%)$.

However, the main concern will be with the divertor inventory and flux of tritium, and it is here that the most gains will probably be made in reduction of tritium hazards. 


\section{Parameters for Hydride Absorption/Desorption \\ Beds for Tritium Recovery}

\section{Table 10-1}

\section{Reactor Power}

Fraction of He coolant Stream Processed

Number of $\mathrm{H}_{e}$ circuits in Reactor

$\mathrm{H}_{e}$ Flow Rate through Processing Bed, Each Circuit

Tritium Inlet Partial Pressure

Tritium Outlet Partial Pressure

Bed Flow Area

Bed Thickness

$\mathrm{H}_{e}$ Superficial Velocity in Bed (no solids)

Fraction of Titanium in Bed Solids

Fraction of Inerts in Bed Solids

Bed Void Fraction

Bed Volume (total, incluoing voids) per circuit

contact Time, -

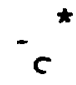

Bed Particle Size

Bed cycle Time, Absorption

Desorption

Tritium Inventory in Beds (12 circuits)

Average Tritium concentration in $\mathrm{Ti}$

$\triangle C / C$

AT During Absorption and Desorption Step
$5000 \mathrm{MW}(\mathrm{th})$

$5 \%$

12

$6.0 \mathrm{~m}^{3} / \mathrm{sec}$

$1 \times 10^{-3}$ Torr

$5 \times 10^{-5}$ Torr

$60 \mathrm{~m}^{2}$

$5 \mathrm{~cm}$

$10 \mathrm{~cm} / \mathrm{sec}$

$1 \%$

$99 \%$

$4.0 \%$

$3.0 \mathrm{~m}^{3}$

$0.5 \mathrm{sec}$

$0.2 \mathrm{sec}$

$2 \times 10^{-2} \mathrm{~cm}$

5 minutes

5 minutes

170.000 curies

$2 \times 10^{-2}$ atom \%

0.27

$\pm 0.010^{\circ}$ 


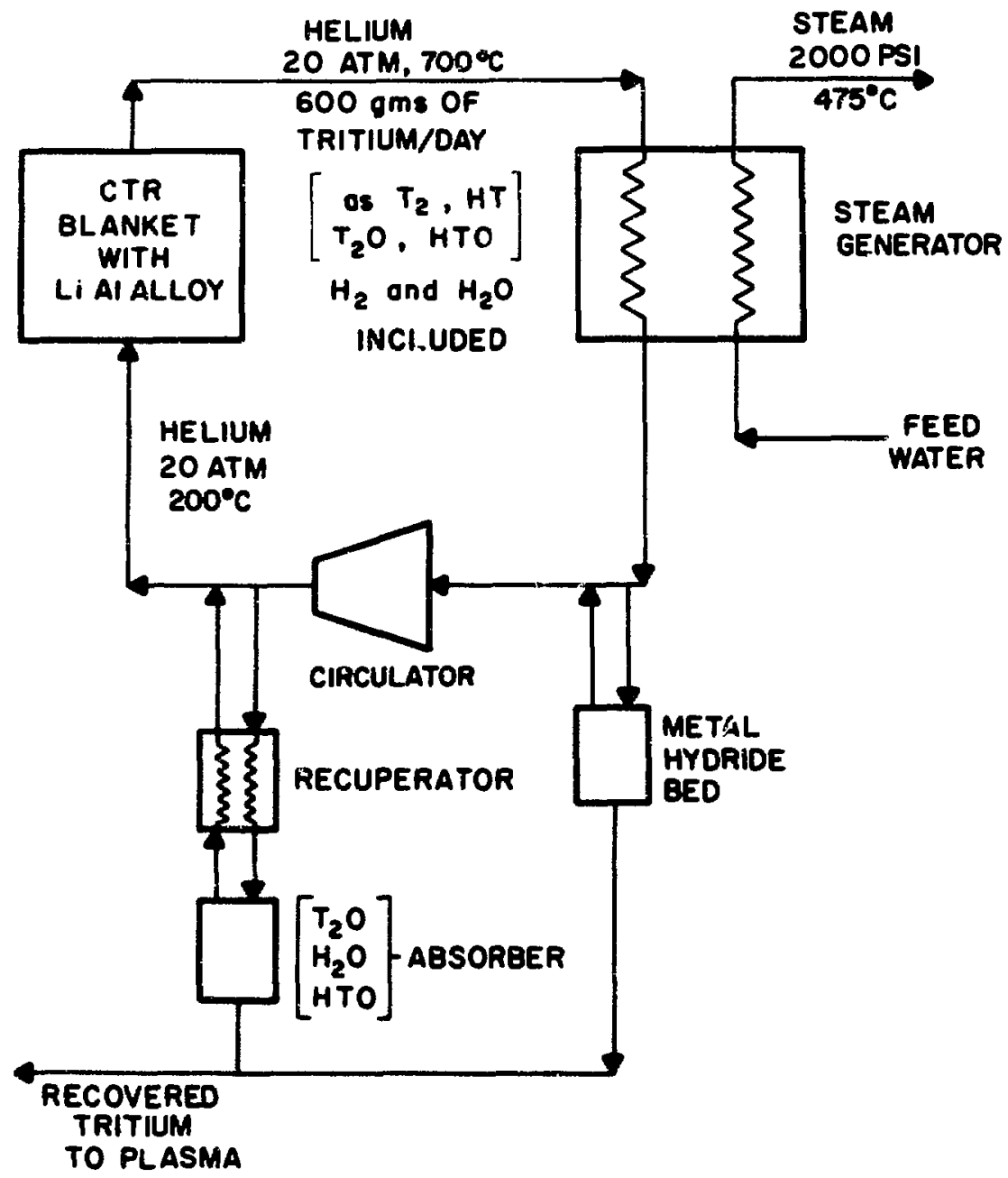

TAITIUM LEAKAgE TO STEAM GEMERATOR, I CURIE/DAY

Figure 10-1 


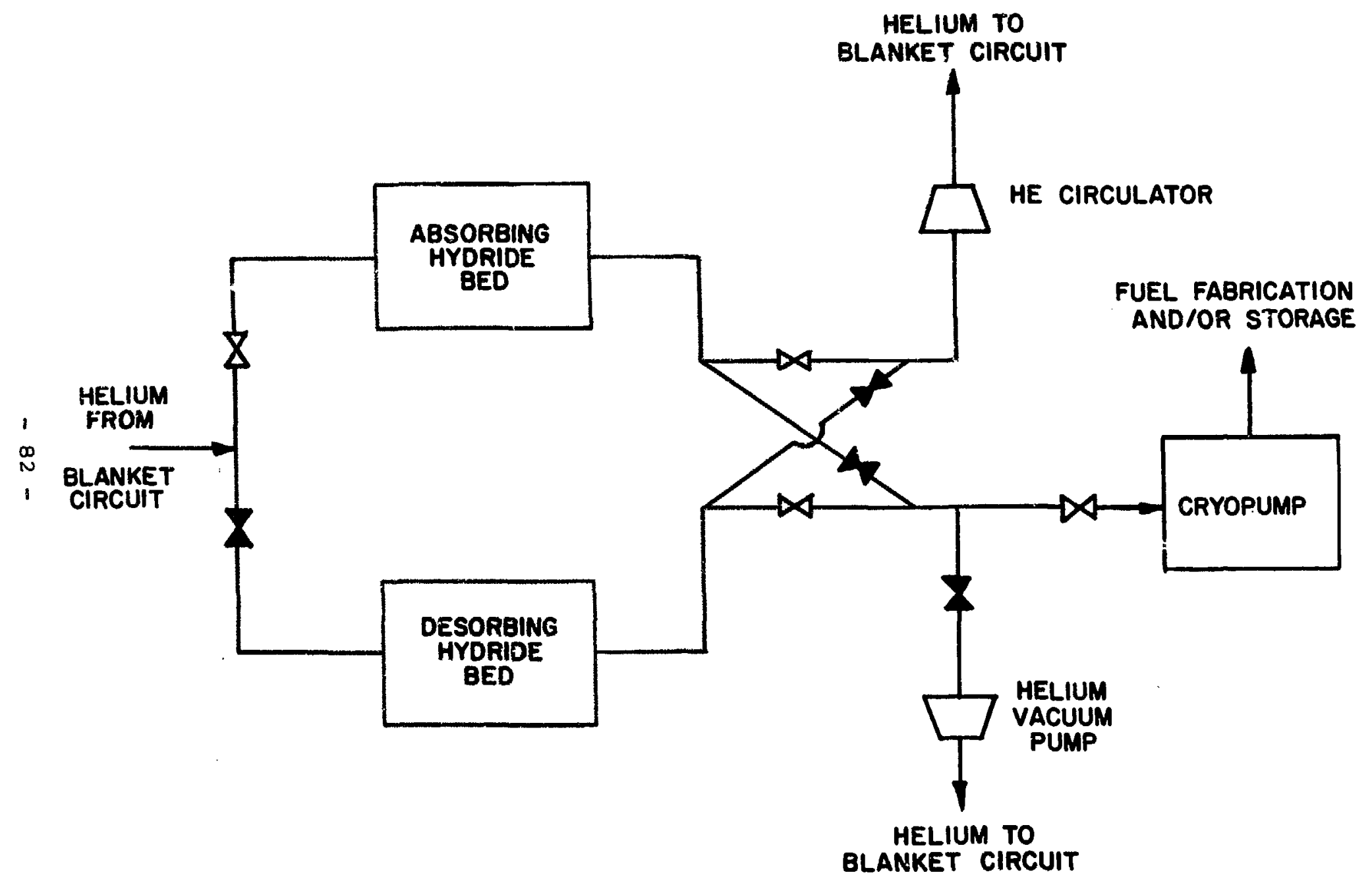

Figure 10-2 


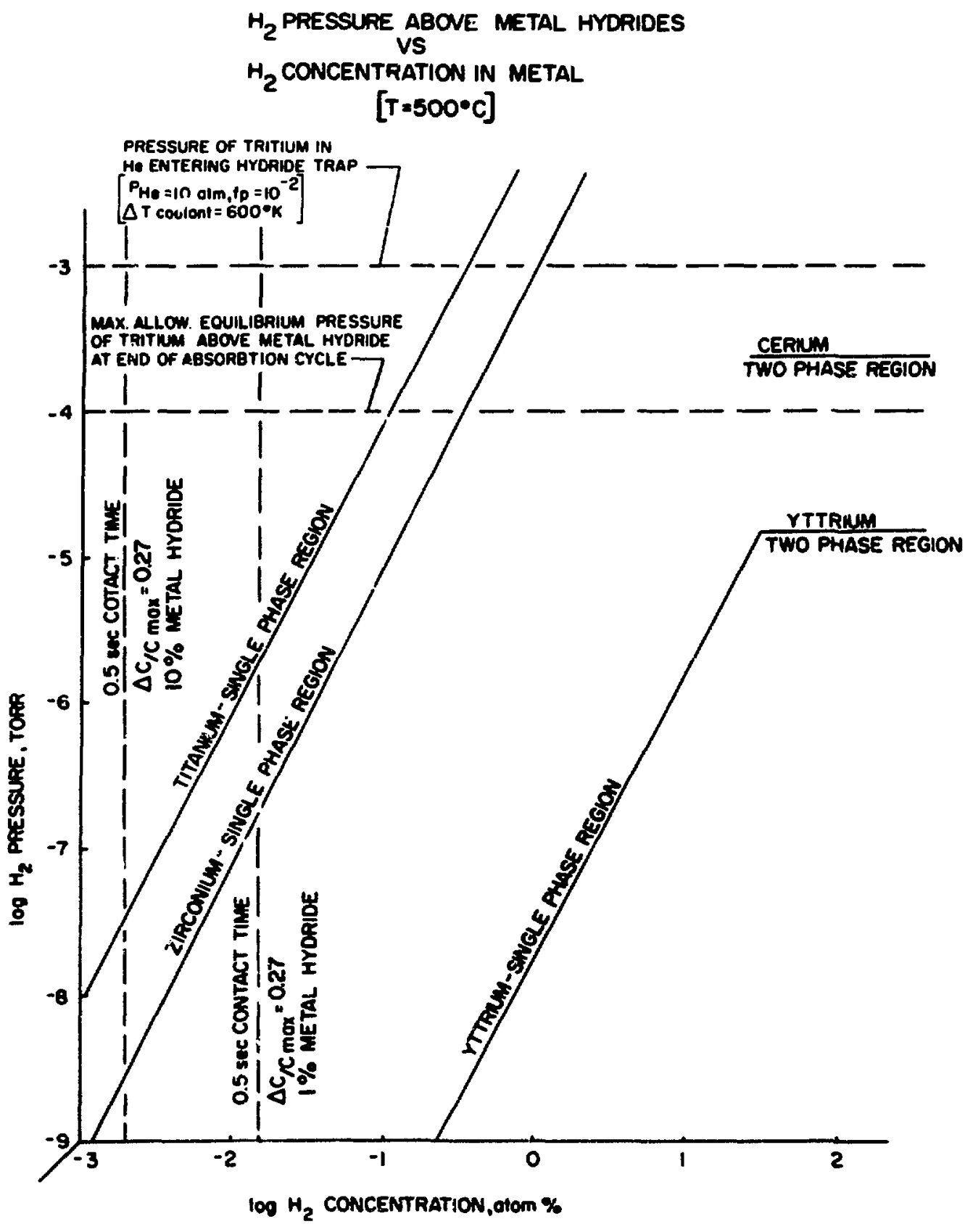

Figure $10-3$ 

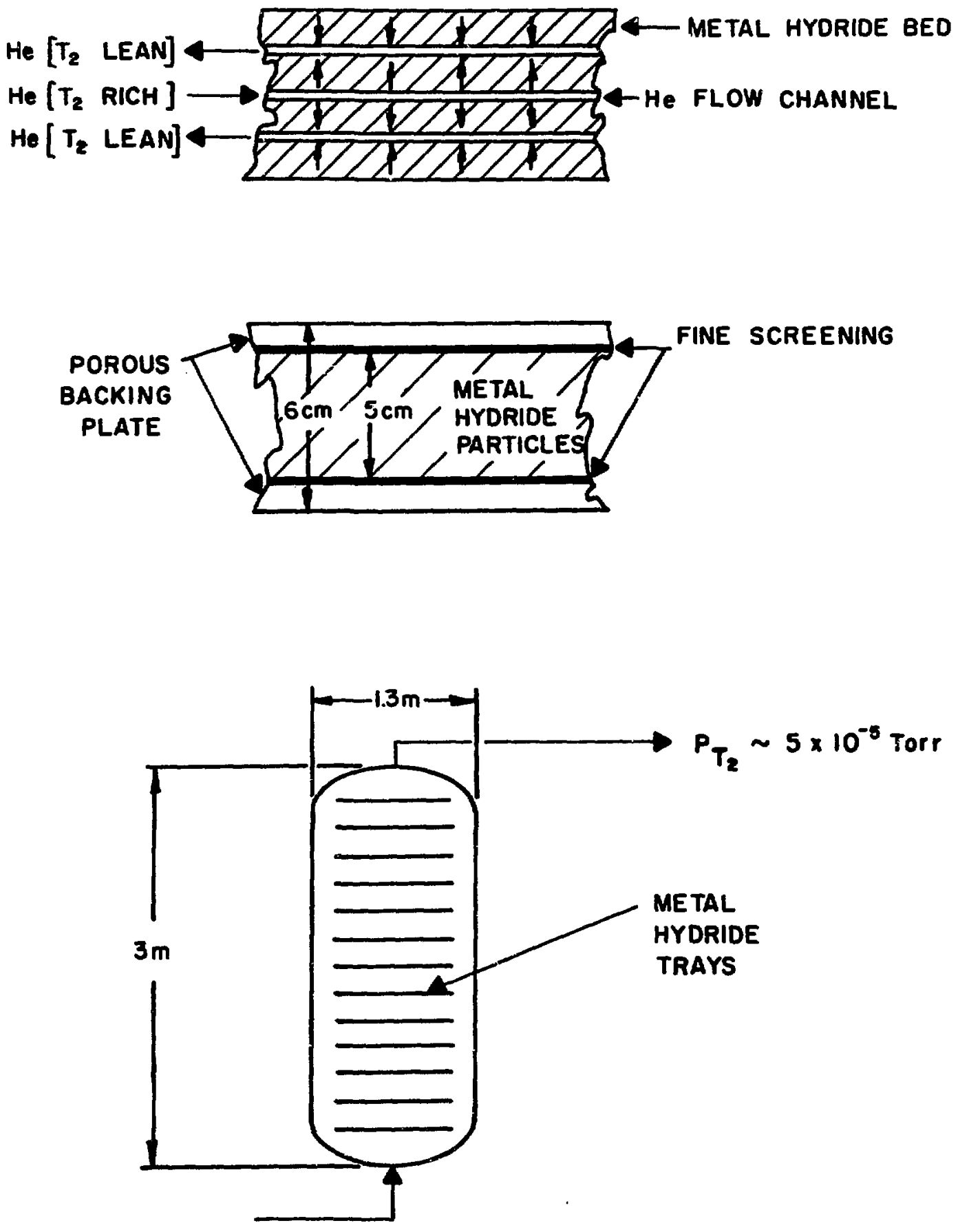

He: $6.0 \mathrm{~m} 3 / \mathrm{sec}$.

$$
\begin{aligned}
& T=500 \circ \mathrm{C} \\
& P=200 \mathrm{am} ; P_{T_{2}} \sim 10^{-3} \text { Torr }
\end{aligned}
$$


TRITIUM MAP FOR 5000 MW(th) REACTOR

WITH SOLID BLANKET

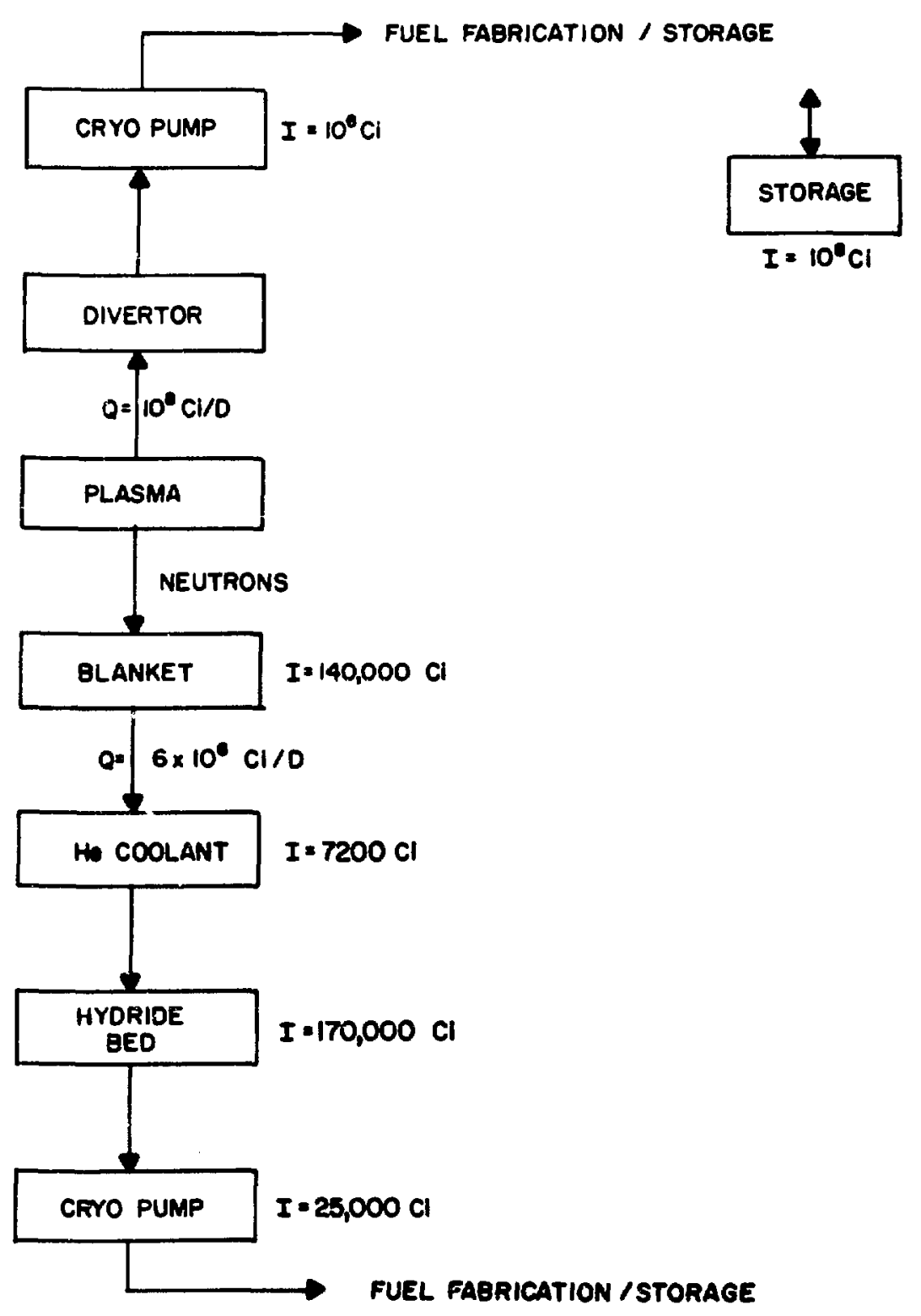

Figure 10-5

$-85-$ 


\section{Blanket Replacement and Processing}

The very low activity CTR contains a blanket made up of about 6000 to 7000 cylindrical modules, each about 100 centimeters overall length and $64 \mathrm{~cm}$ OD. Each module consists of an outer aluminum cylindrical shell with a top and bottom eliiptical head. The aluminum shell and heads contain appropriate holes for passage of c oling water. The bottom head, facing the plasma, is called the first wall.

Each module (see Figure 5-3) contains a neutron multiplying and breeding region of beryllium balls and LiAl pellets, the latter possibly with an aluminum core, an inner graphite plus $\mathrm{LiAlO}_{2}$ region, an outer graphite plus $\mathrm{LiAlO}_{2}$ region, aluminum oxide insulators, aluminum distributor plates, and an aluminum inner shell to direct helium coolant inward along the outer and inner shells, then outward through the neutron multiplying region and the inner and outer graphite structures. The helium and the water circuits remove the useful heat generated by the device and direct this heat to the power plant described elsewhere. The heat results primarily from plasma radiation, high speed neutron slowing down and capture, and gamma radiation.

\subsection{Continuous Tritium Removal}

The tritium which is continually generated by the action of neutrons on the lithium in the LiAl and the $\mathrm{LiAlO}_{2}$ diffuses into the helium coolant. About $5 \%$ of the total helium stream is circulated continuously through titanium beds where titarium tritide is formed. Periodically, these titanium beds are freed of tritium by pumping under reduced pressure and the tritium is recovered and stored as fuel for the CTR, along with natural deuterium. This is a completely closed system and no tritium containing purge gases are released to the atmosphere. A portion of the recovered tritium stream will be sent to an isotope separation process (e.g.. 
liquid hydrogen distillation) to reject protium. This can be combined with the same process for the spent fuel from the divertor.

\subsection{Blanket Module Processing}

Because CF uncurtainty in the effect of the high speed neutrons on material damage of the aluminum shell of the blanket modules, it is assumed that three years of life is all that can be expected until further research on material damage is carried out. To avoid lengthy shutdowns, we assume replacement of one third of the blanket modules each year, or about 2000 to 2500 per year. If the blanket module processing plant can achieve an onstream efficiency of about $80 \%$, it can keep up by processing abisut 8 modules/ day, but to disconnect and remove 2000 to 2500 modules in any reasonable time, and replace and reconinect a like number, is another problem. We assume a 30-d.ay shutdown. After the 10-day cooling off period for $\mathrm{Na}^{24}$, we will have to be able to remove about 115 modules/day and reinstall a like number of new ones in the same period; that is to say, that as one, two, or more modules are removed, a like number of new or reconstituted modules must go back in place at once. This will require an activity level, from the standpoint of manpower, of about 5 modules out and 5 in per hour. This can be achieved by using 5 work crews spaced around the ring, thus allowing each crew one hoix to remove a module and install a new one, all crews working around the clock.

During the 10-day decay period, water can be blown or pumped out. The helium can continue to circulate to remove residual tritium in the module. 'with a mean holdup time of orily an hour or so for tritium in the module, 10 days should reduce tritium inventory in a module to negligible levels (i.e., much less than a millicurie). There are several ways in which to handle the pulling of modules and replacement thereof, and a suitable program will have to be set up to accomplish a complete change in three years. Because 
of the complex system of water and helium piping above the rows of modules, it would appear that such a program would envisage replacement of rows of modules so that fairly long lengths of piping could be removed first from in front of the row.

In the case of occasional failure of a single module, as shown by failure detectors which will be part of the complete plant, the reactor will be shut down. Water and gas lines will be sectionalized as much as possible to isolate the failed module. The failed module (and adjacent ones, if it cannot be completely isolated) is then pumped or blown out and piping cut away which is blocking access to the failed module. The water vapor and helium pumped from the module(s) are sent to contaminated storage. These may be safety released after the txitium is extracted from the helium by titanium beds, and the contamination level made sufficiently small. The failed module(s) are extracted into sealed casks and new ones installed.

\subsection{Elements of Processing Plant}

As mentioned previously, the processing plant should be able to process about 8 modules/day. However, it is likely that considerably more than 8 modules may be in residence in the various parts of the plant at any one time. Furthermore, 2000 to 2500 modules, which it is proposed to remove in a 20-day period during a shutdown, will have to be stored in tight casks awaiting transfer to the processing plant. Since the activity is very low, and decay heat is negligible, these casks could be stored in an area which might consist of a large number of cased 30-inch diameter holes in the grouni, say 100 feet deep, each holding 30 modules. Eighty to 100 such holes would suffice for such storage, but if one wanted to provide for storage of all 7000 modules some 300 such holes would be needed, and the plot plan (Figure 11-1) reflects storage for 7500 modules. 
A first operation on processing a module would be to place it in a chamber, and remove the temporary caps which had been previously installed on the helium inlet and outlet nozzles. It would then be warmed up to some suitable temperature, 600 to $800^{\circ} \mathrm{F}$, while unter a high vacuum to extract any residual tritium. In the absence of any better data, it is assumed that this operation would take about one day; accordingly, either eight or ten chambers would be needed, or a single chamber large enough to hold 8 modules could be used. The helium and tritium pumped out would be passed through titanium beds to absorb the tritium. The helium, if pure enough, can be pumped to storage.

After cooling, a module is now ready for recovery and reconstitition of the beryllium pellets, the LiAl, and the LiAlO ${ }_{2}$ and replacement of graphite.

At this point it should be remarked that whether it will be economically justified to take damaged modules apart and reconstitute or repair the internals sufficiently to justify reuse insj.de a new outer aluminum shell, is not certain. The following assumptions are made as to the probable condition of a module after three years of service, and what parts could probably be reclaimed and/or reconstituted.

1. The outer aluminum shell must be discarded to waste.

2. The inner aluminum she11, because it is subjected only to the very small pressure drop from the coolant inlet to outlet, might be reusable. However, because it must have the top head cut off and a new one welded on, it would be folly at this time to assume that such welding could be successfuI.

3. The beryllium and LiAl pellets are so damaged as to require separation and reconstitution. The lithium burnup requires addition of new lithium. 
4. The inner separator plate is discarded to waste.

5. The graphite in the inner breeding zone, with $\mathrm{LiAlO}_{2}$ filling in certain elements, is assumed to be so damaged that it must be consigned to waste. However, the $\mathrm{IiAlO}_{2}$ may be reclaimed and reconstituted.

6. The graphite in the outer breeding zone is assumed reus able, after the $\mathrm{LiAlO}_{2}$, is emptied out, reconstituted, and enriched in lithium which has burned out.

7. The $\mathrm{Al}_{2} \mathrm{O}_{3}$ insulator in the outer breeding zone is assumed reusable, the insulator in the inner breeding zone is not. After the heating, evacuation, and cooling referred to above, the modules will be transferred to a disassembly cell. Since the activity level will be very low, only minimal shielding (possibly none) will be required. However, because of the possibility of residual tritium, workmen in the vicinity will doubtless need clean air masks, and all ventilation air must be monitored. However, it is expected that the heating and evacuation will essentially remove all of the residual tritium, and the remnants would not constitute an environmental hazard.

\subsection{Steps to be Done on Each Module After Evacuation}

In the discussion below, it is assumed that only dry materials handling chemistry will be used. However, there follows later a short discussion related to wet (aqueous) chemistry if used in certain of the steps. As will be noted many of the steps required in handling a module are purely mechanical.

1. Cut off top head of outer shell and inner shell.

2. Remove upper mechanical support plate. It is assumed this is held by some kind of mechanical latch.

3. Remove upper graphite rods $(50 \mathrm{~cm})$ including the hollow rods containing $\mathrm{LiAlO}_{2}$. Set aside for new module. 
4. Remove upper $\mathrm{Al}_{2} \mathrm{O}_{3}$ insulator and set aside for new module (if reusable). (It should be noted at this point that graphite growth or warping of some kind may take place in both the roas and the $\mathrm{Al}_{2} \mathrm{O}_{3}$, which could preclude reuse in a new module.)

5. Remove lower graphite rods $(20 \mathrm{~cm})$, separate out the graphite for disposal, reclaim $\mathrm{LiAlo}_{2}$ for recycle.

6. Remove lower $\mathrm{Al}_{2} \mathrm{O}_{3}$ insulator and send to disposal. Substantial damage is assumed.

7. Remove distributor plate, and send to disposal.

8. Pour out the $\mathrm{Be}$ and IiAl pellets and separate. Vacuum clean dust at all steps and separate broken or damaged pellets and consign all dust and broken pellets to the reconstitution plant. Since the burnup of Be is quite small, the majority of the pellets may be reusable, provided their structural integrity is still judged satisfactory. Be broken pellets and fines are sent to a reconstitution plant. The IiAl pellets are sent to a reconstitution plant.

Separation of the Be from the LiAl may be a problem, and may necessitate wet or aqueous chemistry. For the moment, it will be assumed that these may be separated by melting the LiAl, which has the lower melting point. The melt can then be enriched in the lithium which has burned out, solidified, and repelletized. The beryllium can be enriched by the amount which has burned out, and by powder metallurgy techniques, sintered and repelletized for reuse.

9. Mix Be and IiAl pellets to make up a proper charge, and pour into the bottom of a new module can. Level off and settle. 
10. Install new lower distributor plate and lock in place.

11. Install new lower $\mathrm{Al}_{2} \mathrm{O}_{3}$ insulator on top of lower distributor plate.

12. Meantime, the $\mathrm{IiAlO}_{2}$ and graphite pellets reclaimed from the lower discarded graphite rods, as well as the same material from the upper graphite rods should be examined for reclamztion and reconstitution. Some $\mathrm{Li}$ will have been burned out, therefore the $\mathrm{LiAlO}_{2}$ will tend to become depleted in lithium if $\mathrm{IiAlO}_{2}$ only is used as makeup. Some means of adding lithium separately will have to be found. For the time being it is assumed that this can be done with wet chemistry, or possibly with dry meicallurgical chemistry such as adding $\mathrm{LiCO}_{3}$ which would subsequently decompose on firing leaving $\mathrm{Ii}_{2} \mathrm{O}$ or $\mathrm{Li}_{2} \mathrm{C}_{2}$ as the lithium enrichment component. All of the $\mathrm{IiAlO}_{2}$ and graphite pellets may be ground up, additional LiAlo and graphite powder added, as well as some pitch to assist in pelletizing. The properly constituted mixture may be pelletized and fired lightly to compact the pellets, which can then be inserted into the lower and Lipper hollow graphite rods and the ends thereof closed with a scresed plug. It would appear that this whole operation could be carried out with only a dust hazard, which by proper vacuum cleaning and filtration could be readily controlled. There might be a slight tritium hazard accompanying the grinding, but this would appear to be minimal.

13. The lower graphite region may now be packed with new rods and reclaimed $\mathrm{IiAlO}_{2}$,

14. Install the old upper $\mathrm{Al}_{2} \mathrm{O}_{3}$ insulator. 
15. Install the upper reclaimed graphite rods and reclaimed $\mathrm{LiAlO}_{2}$.

16. Install the upper holddown plate and lock in place.

17. Weld on the inner upper head and the outer upper head containing the water and helium inlets and outlets, and put temporary caps on the water and helium

18. Pressire test both fluid sets.

19. Transfer to new module storage.

20. Remove from new module storage and install in CTR.

21. Reconnect water and helium manifolds and pressure test.

A plot plan of the entire complex, showing facilities needed for reprocessing and storage, as well as other parts is given in Figure $11-1$. 
The foregoing discussion of the elements of a processing plant and the steps to be carried out are based mostly on "dry" or "metallurgical" type processes and do not involve any steps requiring the handling of the slightly active matcrials in chemical processes involving water acids, or other liquids. The processes envisaged admittedly may pose dust and fume hazards, but it is believed these can be made acceptable.

Chemical processing with liquids is likely to produce quantities of liquid waste which will have to be stored, thus bringing on the usual problems of storage liquids--at least until such liquids can be discharged as meeting toxicity or radiation requirements. However, even if wet chemistry is used, the activity of the solutions will be so low that above ground, easily inspected storage tanks could be used to hold liquid waste.

In the previous descriptions, it was noted that lithium will be incorporated into the graphite structure in the form of $\mathrm{LiAlO}_{2}$ plus graphite pellets in certain of the graphite rods which are hollow. It is believed that the lithium might also be incorporated into the graphite by a wet impregnation process.

Such a process might be accomp? ished by impregnating the graphite rods with a water solution of lithium carbonate, then firing the graphite to drive out the water and decompose the lithium carbonate into $\mathrm{CO}_{2}$ and lithium oxide. This process could be repeated until the desired content of lithium was achieved.

Conceivably, reclaimed graphite rods from a replaced module could be enriched in lithium for further service by a simiiar process. It does not appear that this simple operation would result in any appreciable chemical waste, and may be very advantageous compared to the manufacture and reprocessing of solid $\mathrm{LiAlO}_{2}$. 
The graphite rods in the inner section of a module are assumed to be so damaged that they must be discarded, so re-enrichment of these rods would not be attempted.

\subsection{Reprocessing the Helium coolant}

Impurities in the helium coolant are present as a result of residual air during initial start-up or air inleakage during module replacement, desorption of absorbed impurities from module components, and inleakarje of water from the first wall cooling system or from the steam generator cycle.

Inleakage of small quantities of water can generate corresponding quantities of $\mathrm{CO}, \mathrm{CO}_{2}, \mathrm{CH}_{4}$, and $\mathrm{H}_{2}$ by reaction with the hot blanket graphite. The helium purification system should be able to cope with minor leakage. More serious leakage will probably trigger shutdown for repair.

\subsection{Storage Facilities for Modules}

Adequate storage facilities will be needed for both removed modules, and reclaimed or new modules. It will be assumed that facilities sufficient to accommodate 7500 of each will be needed.

since the modules are cylindrical, the idea of dropping them into cased holes in the ground is appealing. Holes 30-inches in diameter and 100-feet deep would be easy to drill, case, and the casing could be cemented in with standard oil well drilling practices. This was mentioned briefly previously.

Each such hole could hold 25 modules, so 300 holes would be required. An array 18 holes by 18 holes would do. If the holes were located at 6-foot centers, an area of 108 feet by 108 feet would suffice--only about $1 / 4$ acre for a storage field. Lowering and retrieving the modules would be a simple matter. A weatherproof building would be installed over the field. Obviously, the casing would be closed at the bottom and water tight, and anchored 
so as not to float upward due to ground water pressure. Figure 11-2 shows a typical storage well.

11.7 Composition of a Module and Materials That Must Be Consigned to Waste storage

Table 11-1 shows the composition of a single module, the amounts of materials that are reclaimable, lost (i.e., consigned to waste storage) new materials required, the amount consigned to storage per module, the total amount per year, and an estimate of the physical volume of encapsulated waste including concrete encapsulation. It should be noted that the quantities given in this table may not agree exactly with corresponding quantities given in the engineering drawings. However, they give a sufficiently accurate picture of the volumes of waste and pounds of material to be processed.

While one would not encapsulate all waste into a single cube, as postulated in the table, the figures show that probably $1 / 4$ acre of space per year will suffice for waste storage. This need not be buried--it can be above ground on a concrete slab. 
Table 11-1

MATERIAL FLOWS IN MODULE REYROCESSING PLANT

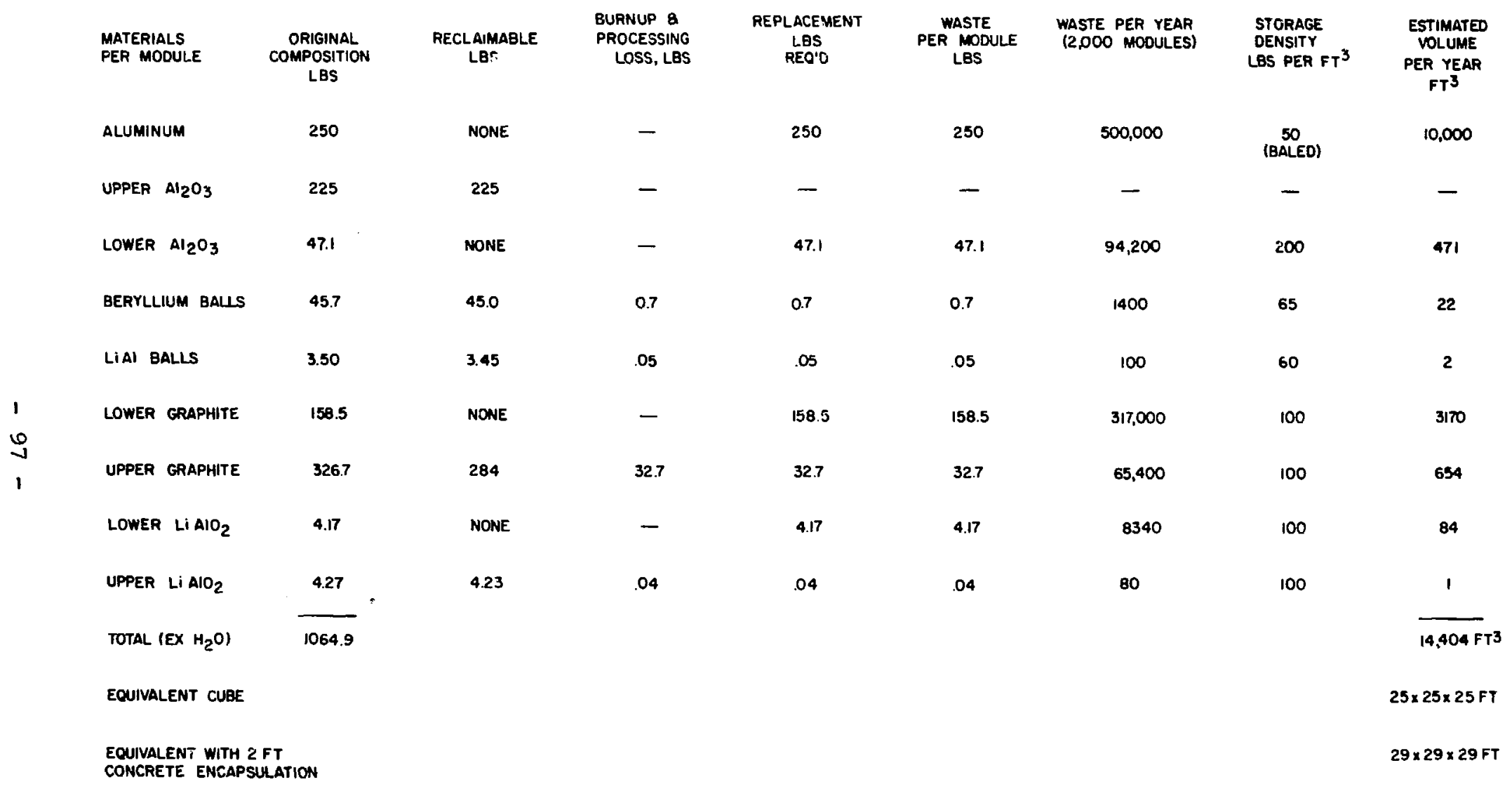




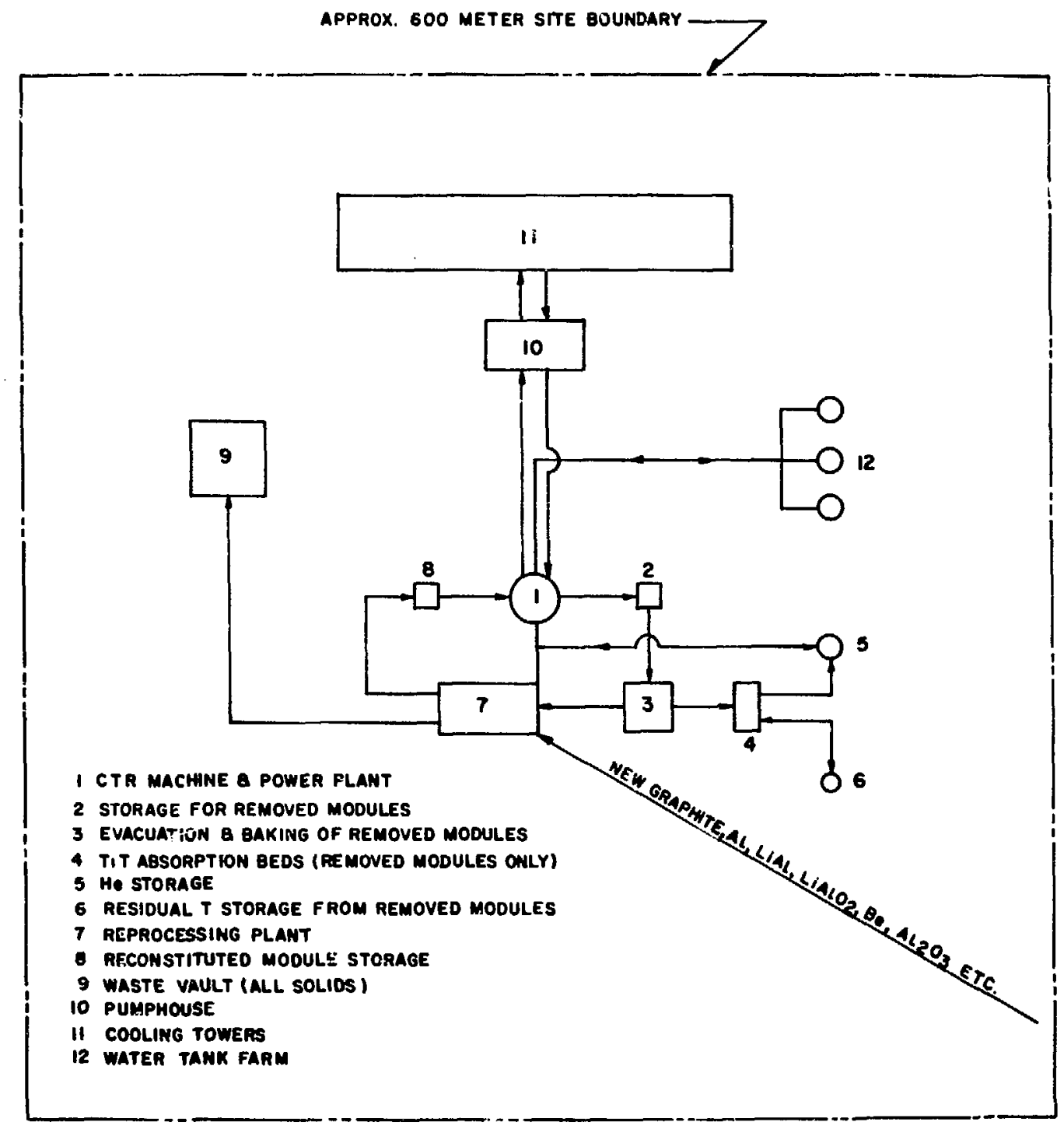

CTR LOW ACTNITY BLANKET SITE PLAN

Figure 11-1 


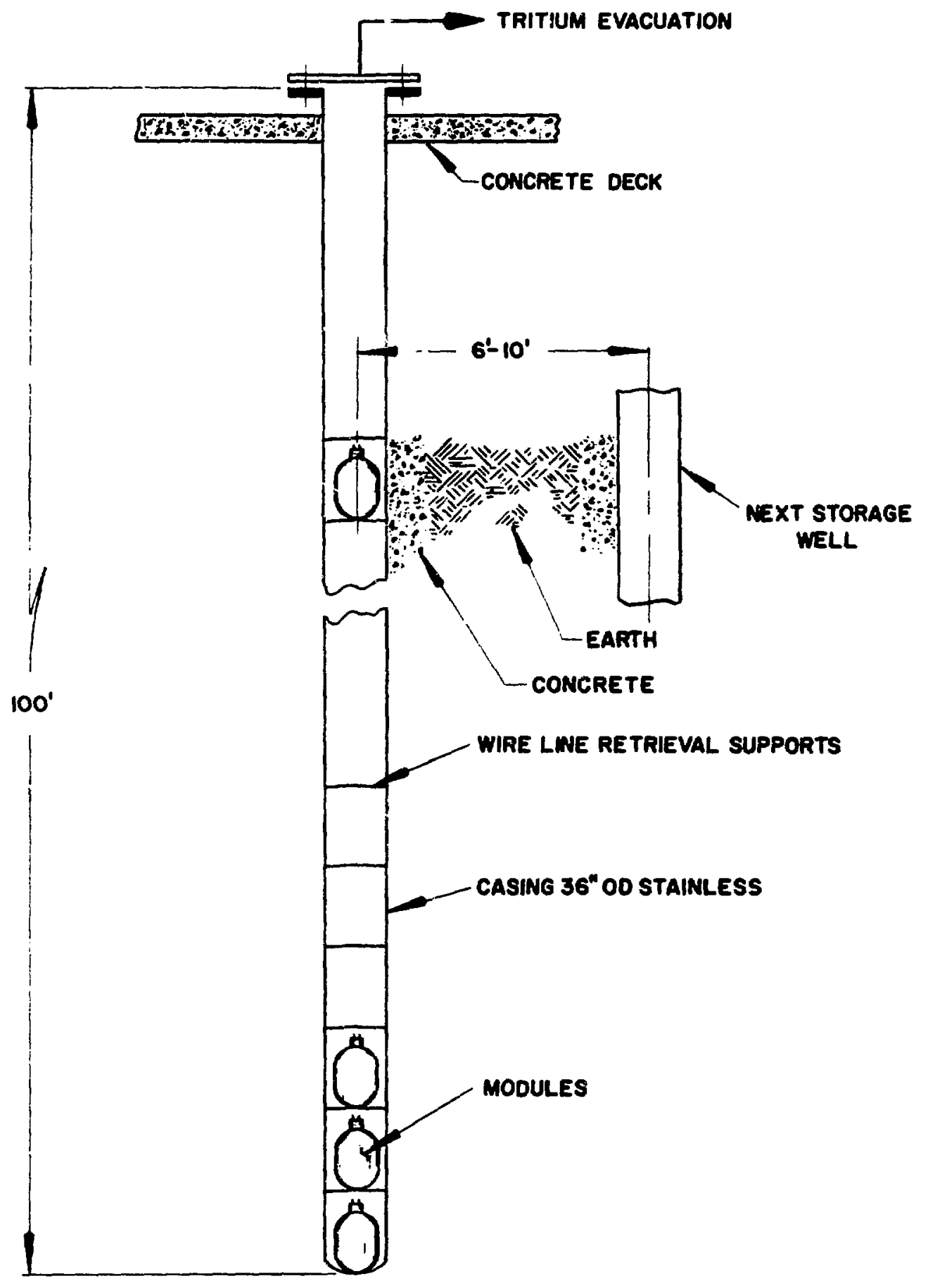

TYPICAL MODULE STORAGE WELL 25 MODULES PER WELL

Figure 11-2 
12. Effluents During Normal Operation and Emergency Containment

It should be recognized that this discussion of normal and emergency containment is based on a preliminary concept of the arrangement of the very low activity blanket on a CTR, and is therefore necessarily sketchy and brief. As the design is further refined, it is to be expected that this section will expand considerably, with appropriate grapins and backup tables of possible activity releases. At the moment, such releases appear minimal.

Analysis of the environmental impact of normal and accidental releases from very low activity fusion blankets are described in an Atomic International study (1). The design considered in the AI study is an early one, but the results are representative.

(a) Normal operation

During normal operation of the CTR with a minimum activity blanket, release of radioactive material should be very low. The tritium which is bred as fuel wiil diffuse into the helium from which it is extracted by the nydride beds. This is a closed system and no effluent can escape the environme t except a small amount of tritium (< 1 curie/day) by normal leaks from the helium coolant circuit.

The very small amount of residual tritium in the blanket modules would be recovered as was described in the processing description in section 10.

There may of course be slight diffusion of tritium into the primary vacuum tank (see Figure 5-1), but this is held at a high vacuum and the output of the vacuum pumps is scrubbed. Any tritium that is recovered would be returned to the process stream. Some tritium will diffuse into the steam systems, but these will be designed for zero blowdown. Blowdown necessary for water chemistry control will be held in closed tanks until cleaned up 
of tritium. In case of a turbine loss of load event, the steam would the condensed and held in closed tanks after the plant is down.

The rate of tritium leakage from the helium coolant into the steam system would be unacceptably high if conventional steel steam generator tubes were used. Tritium barriers of the types considered in other fusion reactor reference designs can be used. These include either making the steam generator tubes with internal layers of a metal (copper or aluminum) with very low tritium permeability $(2,3)$ or spraying an external layer of material with very low tritium permeability layers (tungsten or alumina) on the gas side surface of the tube, assuming no cracks. The tritium leakage into the steam circuit can then be held at $\sim 1$ curie/day.

It may be possible to recover a substantial fraction of this leakage from the steam circuit by tritium cleanup of the water in the closed tanks. This would then result in an amount less than 1 curie/day to be discharged to the environment through leaks, and this should have minimal environmental impact.

The heliurn system will require circulators and compressors, which will likely be equipped with seals, unless the units can be motor drive canned rotor type. While there may be a great development of stzte-of-the-art designs in these respects before a CTR unit is constructed, there will probably still exist the necessity of seals where a helium circulator, for example, is to be driven by a steam turbine. Nevertheless, it is believed that such seals, of a mechanical gas lubricated type, could be so contrived as to accept a high pressure flushing stream of clean helium thus keeping tritium inside while clean helium leaks out. Moreover, even leakage can be gathered up and processed for removal of tritium. 
Approximately $1 \% /$ year of the helium in the closed helium circuit can be assumed to leak into the primary containment building. The tritium leak through this route is then 0.22 curie/diy. Most of this lea'age tritium can be recovered by cleaning of the containment building atmosphere, and the resultant tritium leakage to the environment should be $\ll 1$ curie/day, which is well below the guideline limit of 1 curie/day.

All other radioactive materiais produced (principally $\mathrm{Na}^{24}$ in the aluminum) are locked up solids in the solid blanket materials and ralease of any substantial amount appears very unlikely. in addition to the circulators and compressors mentioned above, the water, steam, and helium systems will require a multitude of valves. It would appear that these need all be single or double bellows sealed, to prevent leakage at stuffing boxes.

(b) Emergency Accident Containment

Since the low activity blanket considered herein contains some 6000 to 7500 individual blanket modules, the most likely accident is failure of a module or group of modules, particularly since each module must look at the direct radiation from the plasma as well as be exposed to the flux of $14 \mathrm{MeV}$ neutrons resulting from the DT reaction.

Failure of a module can release both high pressure hot water and high pressure hot helium into the torus. This will instantly extinguish the plasma, ard other failure detectors will call for an immediate shutdown of all normal operation functions. Since there is no fission product accumulation, and only minimum decay heat, unlike a fission reactor, emergency cooling will be minimum if required at all.

To the extent that helium and water piping can be equipped with emergency isolation valves, failure of a nodule or group of modules can be isolated to minimize the leakage into the torus. 
A failure such as described above would release tritium bearing gases into the torus and primary vacuum tank. These constitute radiological hazards if released, but they could be pumped to contaminated storage for cleanup and safe release. Failure of a module or group of modules could scatter aluminum, beryllium, lithium aluminum, graphite, lithium aluminate, and aluminum oxide into the torus in broken pieces and these might act as missiles, also. However, all radioactivity in these parts, which is very smäll anyway, is locked up in solids and cannot spread around. After a suitable cooling off period for the $\mathrm{Na}^{24}$, the broken materials could be retrieved and sent to chemical processing and waste storage.

There are other types of failures which would have to be considered. For instance, a large helium line could fail, allowing high pressure and possibily hot helium to fill the primary vacuum tank. It should be noted, in passing, that the overall assembly must be so designed that fallure of both s':eam and helium systems into the primary vacuum tank cannot generate a pressure which would rupture the tank. This is a matter of matching relative volumes.

Failures of water lines could introduce hot or cold water into the vacuum tark. Failure of the steam generator tubes in the helium circuit could release high pressure water or steam into the helium system, with possible damage to the circulators. Isolatiori valves can be employed to mitigate some of these effects.

In general, however, it appears that releases of water, steam, or helium into the primary vacuum tank can be handled with conventional pumping, vacuum cleaning, and drying operations, followed by cleanup of the reclaimed fluids. Most of the radio- 
activity would be as tritium, although small quantities of radioactive liquid waste might result, necessitating ion exchange beds, evaporation, or the like. The absence of liquid metals and of fission products after heat mitigates the cleanup problem greatly.

upper limits to the radioactive effluents during accident conditions can be estimated by examining the maximum credible accident, and assuming that the containment system does not function. The solid blanket design should ensure that the failure of the containment system is very unlikely, however, since there are no chances of a lithium fire, or water-lithium reactions.

The total inventory of tritium in the helium coolant circuit is 7200 curies. If all this tritiun were released at ground level, the worst possible case, the integrated dose from tritium at the site boundary would be only $1.7 \mathrm{rem}$, well within the 25 rem limit suggested by $10 \mathrm{CFR}$ 100. (The estimated dose is based on factors developed by the AI environmental study of minimum activity blankets.)(1) If $10 \%$ (14,000 curies) of the total blanket tritium were released, an unlikely event because the tritium is locked in solid compounds, the dose at the site boundary would be only 3 rem. Release of any substantial fraction of the tritium in the titanium trapping beds is even less likely, since this part of the reactor complex is small, and can be easily sectionalized and protected in isolation cells with thick walls.

$\mathrm{Na}^{24}$ is the only radioisotope with significant biological hazard potential if an accident should occur during reactor operation, since the inventory of the other isotopes is very small. Release of any significant fraction of the $\mathrm{Na}^{24}$ to the environment is virtually impossible, since it is dispersed throughout a very large mass of aluminum and aluminum oxide in the blanket. If some pieces of the blanket are dispersed inside the containment 
building, the contained $\mathrm{Na}^{24}$ will decay away in a few days and should not leak out to the environment.

It is believed that a CTR with very low activity blanket, as described in this study, would have a very small impact on the environment from the standpoint of radioactive releases. 


\section{Acknowledgements}

The authors wish to express their deep appreciation to Mr. Locke Bogart, ERDA, Washington, D. C., for many helpful comments and suggestions on this study, to Mr. Wesley Ripperger, for preparation of many of the drawings in this report, to Mr. Warren Johnson, for preparation of the reactor model which greatly aided visualization of the mechanical operations involved, and to Mrs. Judy Ridgeway, Miss Fran Benante, Mrs. Ginny Diebel, and Mrs. Gwen Bergin for preparation and typing of the manuscript. 
References

Section 1

1. Powell, J. R.. et al., "studies of fusion reactor blankets with minimum radioactive inventory and with tritium breeding in solid lithium compounds: A preliminary report." BNL 18236 (1973).

2. POWE11, J. R., et al., "Minimum activity blankets for commercial and experimental power reactors." Proc. 5th Symposium on Engineering Problems of Fusion Research, IEEE publication 73CH08433-NPS, p. 163 (1973).

3. Powell, J. R., et al., "Minimum activity blankets using aluminum structure." Proc. First Topical Meeting on the Technology of controlled Nuclear Fusion, Vol. I, CONF-740402-P1-USAEC, p. 533. (1974).

4. Hopkins, G. R., "Fusion reactor applications of silicon carbide and carbon." Ibid, p. 437.

5. "UWMAK-I - A Wisconsin toroidal fusion reactor design." UWFDM-58, Vol. I., University of Wisconsin (1974).

6. Mang, J., personal communication, Brush Wellman (1974).

7. Wiswall, R. H. and Wirsing, E., "Removal of tritium from solid CTR blanket material." Trans. Am. Nuc. Soc. 19, 20 (1974).

8. Anderson, R. V., "Environmental assessment for fusion reactor utilizing Brookhaven minimum activity blanket." AI-AEC-13136 (1974).

Section 5

1. Kramer, R., personal comnunication, ALCOV (1974).

2. Brown, M. H., et al.. "Aluminum alloys for handling high temperature water." Proc. 16th Annual Conference, National Associatior of Corrosion Engineers, Dallas, Texas, March 14-18, 1960.

3. Kulcinski, G. I. and Conn, R. W., "A helium cooled DT Tokamak power plant-UWMAK-II." Trans. Am. Nuc. Soc. 19, 2 (1974).

4. Powell, J. R. and Lazareth, O., "Fusion blankets with minimum activation and minimum vulnerability to radiation damage." Ibid, p. 17 .

Section 6

1. Drake, M. K., Editor, "Data formats and procedures for the ENDF neutron cross section library." (T-601), ENDF 102, Vol. 1 (October 1970). 
2. Wright, R. Q.. et al., "A program to generate fine group constants and Pn scattering matrices from ENDF/B." ORNL-TM-2679 (September 1969).

3. "Abstracts of the data library pacliages assembled by the radiation shielding information center." ORNL-RSIC- 30 .

4. Joannou, G. D. and Dudek, J. S.. "GAM-II: A $B_{3}$ code $f_{l} r$ the calculation of fast-neutron spectra and associated multigroup constants." GA-4265 (1963).

5. Engle, W. W.. Jr., "A user's manual for ANISN." K-1693 (March 1967).

6. Perlstein, S., "Program THRESH." NNCSC report, September 1971. Distributed by Argonne Code Center, abstract \#504.

7. Ajodon, M. A. and Rossin, R. W., "MACKLIB: 100 group neutron fluence-to-kerma factors and reaction cross sections generated by the MACK computer program from data in ENDF format." ORNLTM-3995.

8. Glasstone, S., "Principles of nuclear reactor engineering." D. Van Nostrand Co.., Inc.. Princeton, New Jersey, pps. 75-79 (1955).

Section 8

1. "Sumnit power station preliminary safety analysis report." Vols. 3 and 6, Delmarva Power and Light Corp. (1974).

2. "Comanche peak steam electric station preliminary safety analysis report." Vol. 5, Texas Utilities Generating company (1974).

3. Kulcinski, G. I. and Conn, R. W., "A helium cooled DT Tokamak power plant-UWMAK-II." Trans. Am. Nuc. Soc. 19. 2 (1974). Section 9

1. "UWMAK-I - A Wisconsin toroidal fusion reactor design." UWFDM-68, Vol. I, University of Wisconsin (1974).

2. Taylor, C., personal comunication, LIL (1974).

Section 10

1. Wiswall, R. H. and Wirsing, E., "Removal of tritium from solid CTR blanket material." Trans. Am. Nuc. Soc. 19, 20 (1974).

2. Gulbransen, E. A. and Andrew, K. F., "Kinetics of the reactions of titanium with $\mathrm{O}_{2}, \mathrm{~N}_{2}$, and $\mathrm{H}_{2} \cdot$ " J. Metals 1. 741 (1949). 
Section 12

1. Anderson, R. V., "Environmental assessment for fusion reactor utilizing Brookhaven minimum activity blanket." AI-AEC-13136 (1974).

2. Mills, R. G., Editor., "A fusion power plant." MATT 1050, Chapt. 15, Princeton Plasma Physics Laboratory (1974).

3. Draley, J. E., Maroni, V.A., and Coultas, T. A., "An environmental impact study of a reference theta-pinch reactor (RTPR)." Proc. of First Topical ANS Meeting on the Technology of Controlled Nuclear Fusion, Vol. I, P. 564, San Diego, California, April 16-18, 1974. 
List of Tables

Table Number

Title

Page

Section 6

6-1

6-2

6-3

6-4

6-5

Section 7

7-1

Section 8

8-1

Summary of Power Cycle Process conditions

64

Section 10

10-1

Parameters for Hydride Absorption/Desorption

80

Section 11

11-1
Blanket composition

41

42

43-4

45

46

Therial Balance for Module and Shield

52

Blanket and Shield Heating
Material Flows in Module Reprocessing Plant 97 
List of Fiqures

Eigure Number

Title

Page

Section 5

5-1

Elevation View of CTR Reactor with Minimum

32

Activity Blanket

5-2

view of Sector $(1 / 12$ th) of Minimum Activity

Reactor

5-3

Cross Section of Blanket Module

34

$5-4$

stress Distribution in Module shell

35

5-5

Cross Section of Flat Sinield Segments

36

5-6

Arrangement of Blanket Modules in Shield

37

Slabs

Section 6

6-1

Simplified Blanket Geometry Used in ANISN

47

Calculations

6-2

Total and $14 \mathrm{MeV}$ Neutron Flux in the Blanket

48 and Shield

$6-3$

Activation of Blanket and Shield After Reac- 49 tor Shutdown

\section{Section 7}

$$
7-1
$$

Temperature and Pressure Drop Profile in

7-2

Typical Blanket Module ( $\mathrm{H}_{2} \mathrm{O}$ Circuit)

Temperature and Pressure Drop Profile in

Typical Blanket Module (He Circuit)

Section 8

\section{8-1 \\ 8-2}

$8-3$

8-4

Section 10

10-1
Flow sheet for Power Generation Cycle

65

Effect of Relative Input to $\mathrm{H}_{2} \mathrm{O}$ circuit in Blanket on overall Thermal Efficiency

66 Effect of Flash Drum Temperature on Power Cycle Performance

Effect of Aluminum First wall Temperature on Power Cycle Performance

Overall Flow sheet for Tritium Recovery

81 

Hydride Beds

$\mathrm{H}_{2}$ Pressure Above Metal Hydrides vs. $\mathrm{H}_{2}$

$10-4$

Schematic Illustration of Hydride Absorption/Desorption Beds for Tritium Recovery from Helium Coolant

$10-5$

Tritium Map for $5000 \mathrm{MW}$ (th) Reactor with solid Blanket

\section{Section 11}

11-1

CTR Low Activity Blanket site Plan

$11-2$

Schematic Illustration of Module Storage

99 Well 